\title{
The physical modeling and aerodynamics of turbulent flows around horizontal axis wind turbines
}

DOI:

10.1016/j.energy.2016.11.060

\section{Document Version}

Accepted author manuscript

Link to publication record in Manchester Research Explorer

\section{Citation for published version (APA):}

Abdulqadir, S., lacovides, H., \& Nasser, A. (2017). The physical modeling and aerodynamics of turbulent flows around horizontal axis wind turbines. Energy, 119, 767-799. https://doi.org/10.1016/j.energy.2016.11.060

\section{Published in:}

Energy

\section{Citing this paper}

Please note that where the full-text provided on Manchester Research Explorer is the Author Accepted Manuscript or Proof version this may differ from the final Published version. If citing, it is advised that you check and use the publisher's definitive version.

\section{General rights}

Copyright and moral rights for the publications made accessible in the Research Explorer are retained by the authors and/or other copyright owners and it is a condition of accessing publications that users recognise and abide by the legal requirements associated with these rights.

\section{Takedown policy}

If you believe that this document breaches copyright please refer to the University of Manchester's Takedown Procedures [http://man.ac.uk/04Y6Bo] or contact uml.scholarlycommunications@manchester.ac.uk providing relevant details, so we can investigate your claim.

\section{OPEN ACCESS}




\title{
The physical modeling and aerodynamics of turbulent flows around horizontal axis wind turbines
}

\author{
Sherwan A. Abdulqadir Hector Iacovides \\ Adel Nasser \\ Turbulence Mechanics Group, School of MACE, University of Manchester, Manchester, UK
}

This paper aims to assess the reliability of turbulence models in predicting the flow fields around the horizontal axis wind turbine (HAWT) rotor blades and also to improve our understanding of the aerodynamics of the flow field around the blades. The simulations are validated against data from the NREL/NASA Phase VI wind turbine experiments. The simulations encompass the use of twelve turbulence models. The numerical procedure is based on the finite-volume discretization of the 3D unsteady Reynolds-Averaged Navier-Stokes equations. The resulting simulations are compared with the full range of experimental data available for this case.

The main contributions of this study are in establishing which RANS models can produce quantitatively reliable simulations of wind turbine flows and in presenting the flow evolution over a range of operating conditions. At low (relative to the blade tip speed) wind speeds the flow over the blade surfaces remains attached and all RANS models tested return the correct values of key performance coefficients. At higher wind speeds there is circumferential flow separation over the downwind surface of the blade, Moreover, within the separation bubble the centrifugal force pumps the flow outwards, which at the higher wind speeds suppresses the formation of the classical tip vortices. RANS models which do not rely on the linear effective viscosity approximation generally lead to more reliable predictions at higher wind speeds. By contrast some popular linear effective viscosity models perform the poorest over this complex flow range. Finally all RANS models are also able to predict the dominant (lowest) frequency of the pressure fluctuations.

\section{Introduction}

Renewable sources of energy (such as wind, solar and wave) continue to attract tremendous interest because of the negative environmental impact of the traditional energy sources that are based on fossil fuels, such as coal, oil and natural gas. One of the most developed and cost-effective renewable energy sources is wind energy, because of its environmentally friendly and economically low cost. The global cumulative capacity of wind power installations approached $198 \mathrm{GW}$ in 2010, while this capacity increased dramatically to reach to $370 \mathrm{GW}$ by the end of 2014 [1]. This demand has speeded up the development of wind turbine technologies. The Horizontal Axis Wind Turbine (HAWT) emerged as the most popular in today's market, because of its high efficiency.

Numerous efforts have been directed towards the improvement of the design of wind turbines and the efficiency of the rotor blades. Early investigations relied mainly on the use of the Blade Element Momentum (BEM) approaches and the experimental data for lift and drag coefficients to investigate the complex flow around the blade [2,3,4]. BEM approaches fail to resolve the 3D flow around the rotor blade because they use 2D airfoil data and empirical models to capture the 3D influences [5]. Moreover these models are limited by impractical assumptions such as uniform wind and steady flow [6]. Although the data measurements are able to provide precise and reliable performance parameters of the wind turbine, the interior details of the flow around the blade remain unexplored. Consequently, the development of more effective designs remains a slow process based on trial and error. Therefore to overcome the restrictions of the experimental methodology, the current study employs computational fluid dynamics (CFD) simulations to investigate the aerodynamics of the flow field around the rotor blades.

Most researchers to date have been validating the numerical approaches through comparing with the National Renewable Energy Laboratory (NREL) Unsteady Aerodynamics Experiment (UAE) [7,8]. These experiments give extremely precise measurements of the flow around the modified Grumman $20 \mathrm{~kW}$ two-bladed wind turbine, performed in the wind tunnel at NASA Ames. These highly significant measurements are obtained from the Phase VI of the experiments.

A variety of CFD techniques have been used to predict the flow field around the wind turbine blades and their efficiency. Duque et al [9] studied the aerodynamic performance of the Phase VI rotor. They applied the Baldwin and Barth algebraic turbulence model [10]. The authors compared the RANS Baldwin-Barth predictions with those of a vortex lattice code. The resulting comparisons demonstrated that introduction of the RANS solver improved the predictions in terms of aerodynamic power, lifting force and surface pressure coefficient. In a parallel study of the Phase II rotor, Duque et al [11] employed three numerical methods, the blade element momentum (BEM) method, by using YAWDYN/AERODYN code, the vortex lattice (VL) method using CAMRAD II code and the Reynolds-averaged Navier-Stokes method (RANS) by using OVERFLOW code. In the RANS method, the boundary layer turbulence was modelled using two algebraic turbulence models: Baldwin-Lomax (Baldwin and Lomax [12] and Baldwin-Barth [10] models. At the lower wind speeds, the predictions of the three approaches for the aerodynamic power and the sectional normal force coefficient agreed well with the NREL experiments. At higher wind speeds, however, they showed notable differences. 
Sorensen et al [13] tested the use of RANS in the prediction of the Phase VI rotor for several wind speeds using the SST( $k-$ $\omega)$ model. Qualitatively the model was able to predict the experimental trend. Quantitatively, on the other hand, the $\operatorname{SST}(k-\omega)$ model showed strong deviations from the experiments at intermediate and high wind speeds. Time-dependent calculations of the NREL Phase VI wind turbine at full-scale were presented by Li et al [14]. RANS and Detached Eddy Simulation (DES) were used to account for the effects of turbulence. They employed the SST $(k-\omega)$ model and simulated cases with fixed and variable pitch angles. Comparisons between RANS and DES calculations at low and high wind speeds were performed. At low wind speeds both approaches predicted essentially the same flow around the blades. At high wind speeds, however, the predicted flow by both approaches changed dramatically, with the DES capturing the highly unsteady separated bubbles and the $\operatorname{SST}(k-\omega)$ model failing to reproduce most of them. Sezer-Uzol and Long [15] studied the effect of wind speed and yaw angle on the flow around the Phase VI rotor using large eddy simulation (LES) approach. The results of the local surface pressure coefficient showed relatively high deviations from the NREL experiments. Van Rooij and Arens [16] examined the phenomenon of augmented lift caused by the blade rotation at different wind speeds using incompressible, steady state, RANS, with the SST $(k-\omega)$ model. The results demonstrated that the augmented lift was caused by radial flow in the separated regions.

Four further and relatively recent numerical studies, Yu et al [17], Moshfeghi et al [18], Lanzafame et al. [19] and Lynch and Smith [20] all focussed on the use of the SST $(k-\omega)$ model in flow predictions past the NREL Phase VI rotor. In the last of these studies a hybrid RANS/LES method was also employed. In all these studies the SST-based RANS computations were shown to deviate from the NREL measurements at high wind speeds. The hybrid RANS/LES method performed better than the SST-based RANS, but was still not in full agreement with the experimental data at high wind speeds.

Finally Tacho et al [21] explored the effectiveness four RANS models, the Spalart-Allmaras, the standard high-Reynoldsnumber $k-\varepsilon$, the $k-\varepsilon$ renormalization group (RNG), and the SST $(k-\omega)$ models. They found that even at relatively low wind speeds the predictions of these models strongly deviated from the NREL data.

The majority of earlier numerical studies on this topic have confined themselves to a limited number of turbulence models. There has been little attempt to explore if, for example, introduction of stress transport models or non-linear eddy viscosity models can improve the quantitative accuracy and reliability of the predictions, or indeed whether the use of high-Reynoldsnumber models can reduce computational costs and increase turnover.

The primary objective of this paper is to advance our understanding of time-dependent flow past rotating HAWT rotor and to assess the effectiveness of turbulence models in capturing the aerodynamics of the flow field around the turbine blades. The investigation tests twelve different turbulence models representing low- and high-Reynolds-number, linear and nonlinear eddy-viscosity models, and Reynolds stress transport models. The simulations include cases with constant blade pitch angle $\left(3^{\circ}\right)$, rotational speed of approximately $72 \mathrm{RPM}$, and wide range of wind speeds, which in turn leads to a wide range of tip speed ratios. All computations are performed using the finite volume flow solver, STAR CCM+. Extensive comparisons with all available NREL experimental data are carried out to fully assess the effectiveness of the turbulence models included in this study and to understand the reasons why. In order to achieve this objective, detailed comparisons of a number of flow field properties around the blade surface are carried out, including plots of the relative velocity field. These also further advance our understanding of the flow dynamics. The use of effective dimensionless operating parameters is also explored.

\section{Mathematical Formulation and numerical methods}

The computations of turbulent flow past the rotating blade of the wind turbine are carried out using commercial CFD code STAR-CCM+. In this code the finite volume approach is utilized to replace, through the process of discretization, each of the differential flow transport equations, with a set of algebraic equations, one for each control volume, which can then be solved numerically. At each iteration the transport equations for the momentum components in the three directions are first solved sequentially to update the velocity field and the SIMPLE algorithm, Patankar and Spalding [22], pressure correction equation is subsequently used to update the pressure field and correct the velocity field to satisfy the mass conservation. Other transport equations, for the turbulence field, are subsequently solved. Convection terms are discretized with a secondorder upwind scheme. A second-order temporal scheme discretization is employed for transient terms.

The resulting discretized equations have been solved using the Hybrid Gauss-LSQ gradient method and an Algebraic Multigrid (AMG) linear solver. The solution convergence criteria have been based on (i) the values of the residuals for the continuity, x-, y-, z-momentum equations; (ii) Global parameter plots, for example, in the current study the aerodynamic torque and thrust are to check if their average values are approximately constant for the last rotor blade cycles (at least for 5 cycles); and (iii) local parameters such as the surface pressure coefficient at a certain point on the blade surface.

\subsection{Governing Equations and Turbulence modelling}

The conservation principles of mass and momentum are applied to an infinitesimal element of fluid to derive the NavierStokes (NS) equations. The 3D incompressible Navier-Stokes (NS) equations with no heat addition, no mass diffusion and 
no body forces, are expanded through decomposing the velocity field into the mean and fluctuating velocity components:

$$
\underbrace{U_{i}^{\prime}}_{\text {instant }}=\underbrace{U_{i}}_{\text {mean }}+\underbrace{u_{i}}_{\text {fluctuating }}
$$

Few mathematical manipulations result in the Reynolds-averaged form of the Navier-Stokes equations (or RANS equations) in an inertial frame of reference using suffix notation as follows:

$$
\begin{aligned}
& \frac{\partial U_{i}}{\partial x_{i}}=0 \\
& \frac{\partial\left(\rho U_{i}\right)}{\partial t}+\frac{\partial}{\partial x_{j}}\left(\rho U_{j} U_{i}\right)=-\frac{\partial P}{\partial x_{i}}+\frac{\partial}{\partial x_{j}}\left(2 \mu S_{i j}-\rho \overline{u_{i} u_{j}}\right) \\
& S_{i j}=\frac{1}{2}\left(\frac{\partial U_{i}}{\partial x_{j}}+\frac{\partial U_{j}}{\partial x_{i}}\right)
\end{aligned}
$$

where $\rho$ is the density, $P$ is the pressure, $\mu$ is the molecular viscosity coefficient, $S_{i j}$ is the mean strain rate tensor, and the index $\mathrm{i}, \mathrm{j}, \mathrm{k}=1,2,3$ refers to the components in each coordinate direction. As can be seen in equation (3), the RANS form of the momentum equations includes an additional group of terms, namely spacial gradients of the Reynolds-stress tensor, $\tau_{i j}=-\rho \overline{u_{i} u_{j}}$, which is symmetric. Since in the RANS form of the Navier-Stokes equations there are more unknown variables than equations additional equations need to be introduced to determine the variation of the six components of the Reynolds stress tensor. While, as will be discussed later, transport equations can be derived for the Reynolds stresses, these include further unknown terms, so at some stage an approximation needs to be introduced. A variety of mathematical models for the Reynolds stresses, known as turbulence models, have consequently been developed, which involve different levels of approximation and result in flow predictions of varying quality. An extensive comparison of the effectiveness of different types of turbulence models over a range of non-equilibrium flows is provided in the recent study of Klein et al. [23]. The predictive advantages and disadvantages of the different model types are demonstrated.

Twelve turbulent models, grouped in Table 1, are briefly described in the following subsections. Only their major features are included in the main body of the paper. More details can be found either in the Appendix or in the original references.

\subsubsection{Eddy-Viscosity Models (EVM)}

This group of models employs the Boussinesq hypothesis [32] to model the Reynolds-Stress tensor algebraically based on an analogy between the Reynolds-Stress and the strain rate tensors as displayed in equation (5). A linear relationship is produced between the Reynolds-Stress and the mean strain rate tensors. The modelling of the eddy viscosity can involve: zero, one or two equation models, the numbers denoting the number of transport equations, employed for this purpose. These are transport equations for turbulent quantities such as the turbulent kinetic energy $\mathrm{k}$ and a second turbulent parameter, which essentially determines the turbulent length-scale, such as the dissipation rate of the turbulent kinetic energy, $\varepsilon$, or the ratio of $k / \varepsilon$, denoted by $\omega$. All of these models involve the same basic assumption that the eddy viscosity $\mu_{t}$ is isotropic, meaning that the ratio between Reynolds stress and mean strain rate $S_{i j}$ is constant in all directions. This assumption is not valid in most complex flows and it can consequently lead to incorrect predictions [33].

$$
\tau_{i j, L}=-\rho \overline{u_{i} u_{j}}=2 \mu_{t} S_{i j}-\frac{2}{3} \rho k \delta_{i j}
$$

where $\tau_{\mathrm{ij}, \mathrm{L}}$ is the Reynolds stress and the sub L refers to linear, $\mu_{t}$ is the eddy viscosity which is a hypothetical property of the flow and requires modelling, $\delta_{i j}$ is the Kronecker delta equal to 1 if $i=j$ and 0 otherwise.

Two-equation models are more complex and consequently more expensive than the one- or zero- counterparts, but far more general, because they do not involve the arbitrary prescription of a length scale. They have consequently established themselves as the models most widely used. The most commonly used two-equation model is the $(k-\varepsilon)$ version, originated by Jones and Launder [24], usually with coefficients and damping functions proposed by Launder and Sharma [34]. This model involves transport equations for the turbulent kinetic energy $k$ and its dissipation rate $\varepsilon$. A wide range of other two-equation models are available in the scientific literature, such as those of, Menter [27], Lien et al [25], Shia et al [26], and Goldberg and Apsley [35]. The major differences between them are in the selected turbulent quantities in which the transport equations are solved for, and whether the model coefficients are constant or functions of local flow properties, or the wall distance. In addition, the low-Reynolds-number models include different types of damping functions and terms which become active only when local turbulent length scales small enough for the viscous effects to be significant, as is the case in the wall viscous sub-layer. The two-equation models generally give acceptable results for shear dominated flows where the normal turbulent stresses are not influential. Their predictions of complex flows are not always successful.

The linear two-equation models (linear eddy-viscosity models) fail to capture the subtle connections between Reynolds stresses and production of turbulent kinetic energy, due to the anisotropic nature of the normal stresses. Therefore, nonlinear two-equation models (non-linear eddy-viscosity models) have been developed. A number of quadratic and cubic formulations have been proposed such as Lien et al [25], Speziale [36], and Craft et al. [37]. They aim to improve model 
predictions through sensitising the Reynolds stresses to strain and vorticity invariants and making the algebraic expressions for the Reynolds stresses either quadratic, or cubic in terms of the strain rate tensor. Moreover the constant $C_{\mu}$, which appears in the equation for the turbulent viscosity, is made a function of the dimensionless strain rate, a feature demonstrated in Klein et al. [23] to improve the predictive capabilities of the model in regions subjected to strong shear.

For all turbulence models, the flow is characterised using time and length scales, usually $k / \varepsilon$ or $\sqrt{v / \varepsilon}$ for the time scales of the large, energy-containing and the small, dissipative scales respectively and $k^{3 / 2} / \varepsilon$ for the length scale of the large eddies. The eddy-viscosity can be written as:

$$
\mu_{t}=\rho c_{\mu} f_{\mu} k T
$$

where $T=\max \left(k / \varepsilon, \mathrm{C}_{\mathrm{t}} \sqrt{v / \varepsilon}\right)$ is the turbulent time scale, $c_{\mu}$ is a coefficient, and $f_{\mu}$ is a damping function employed in low-Reynolds-number models and for High-Reynolds-number models $f_{\mu}=1$.

The quadratic and cubic non-linear formulations proposed by Lien et al. [25] are given in equations (7) and (8) respectively:

$$
\begin{gathered}
\tau_{i j, Q}=-\rho \overline{u_{i} u_{j}}=\tau_{i j, L}-4 C_{1} \mu_{t}(k / \varepsilon)\left[S_{i k} S_{j k}-(1 / 3) S_{k l} S_{k l} \delta_{i j}\right]+4 C_{2} \mu_{t}(k / \varepsilon)\left(\Omega_{i k} S_{k j}+\Omega_{j k} S_{k i}\right) \\
+4 C_{3} \mu_{t}(k / \varepsilon)\left[\Omega_{i k} \Omega_{j k}-(1 / 3) \Omega_{l k} \Omega_{l k} \delta_{i j}\right]
\end{gathered}
$$

where $\Omega_{i j}=\frac{1}{2}\left(\frac{\partial U_{i}}{\partial x_{j}}-\frac{\partial U_{j}}{\partial x_{i}}\right)$ is the mean vorticity tensor.

For the linear EVM, the Reynolds stresses are computed by equation (5), for the Lien quadratic non-linear EVM by equation (7), and for the Lien cubic non-linear EVM by equation (8).

As most of the EVM models used in this investigation contain two transport equations, the turbulent quantities are turbulent kinetic energy $\mathrm{k}$ and its dissipation rate $\varepsilon$ or the specific dissipation rate $\omega$. The general transport equations for $\mathrm{k}$ and $\varepsilon$ are:

$$
\begin{aligned}
& \frac{\partial(\rho k)}{\partial t}+\frac{\partial\left(\rho U_{j} k\right)}{\partial x_{j}}=\frac{\partial}{\partial x_{j}}\left[\left(\mu+\frac{\mu_{t}}{\sigma_{k}}\right) \frac{\partial k}{\partial x_{j}}\right]+P_{k}-\rho \varepsilon \\
& \frac{\partial(\rho \varepsilon)}{\partial t}+\frac{\partial\left(\rho U_{j} \varepsilon\right)}{\partial x_{j}}=\frac{\partial}{\partial x_{j}}\left[\left(\mu+\frac{\mu_{t}}{\sigma_{\varepsilon}}\right) \frac{\partial \varepsilon}{\partial x_{j}}\right]+\frac{1}{T}\left[C_{\varepsilon 1} P_{k}-\rho C_{\varepsilon 2} \varepsilon\right]
\end{aligned}
$$

The term $P_{k}$ represents the production of turbulent kinetic energy and is described as:

$$
P_{k}=-\rho \overline{u_{i} u_{j}} \frac{\partial U_{i}}{\partial x_{j}}
$$

Various categories of the linear and non-linear EVM's are briefly described as follows:

$\boldsymbol{S}(\boldsymbol{k}-\boldsymbol{\varepsilon}) \mathbf{H R}$ [24], $(\boldsymbol{k}-\boldsymbol{\varepsilon})$ Lien HR Non-linear Quadratic [24, 25], $(\boldsymbol{k}-\boldsymbol{\varepsilon})$ Lien HR Non-linear cubic [17, 25] The original version of the two-equation model is the $(k-\varepsilon)$ model which includes transport equations, equations (9) and (10), for $k$ and $\varepsilon$ respectively, proposed by Jones and Launder [24], with coefficients given by Launder and Sharma [34]. The Reynolds stresses are computed from equation (5), and the eddy viscosity by equation (6), using constant $c_{\mu}$. The $\mathrm{S}(k-\varepsilon) \mathrm{HR}$ model avoids the need for integrating the transport equations right up to the wall by taking advantage of wall function, usually log-law approximation, which relates the shear stress at the wall to the mean flow quantities, such as velocity and turbulent kinetic energy, at the nodes close to the wall. Therefore the need for a fine resolution of the nearwall regions is avoided.

For non-linear EVMs [25], the two high-Re versions, quadratic and cubic, $(k-\varepsilon)$ LHRNQ and $(k-\varepsilon)$ LHRNC, the production due to the non-linear parts $\left(\tau_{i j, Q}\right.$ and $\left.\tau_{i j, C}\right)$ of the stress is included. These parts include non-linear terms of the strain and rotation tensors that are used to account for anisotropy and sensitize the turbulent flow to streamline curvature and rotation. The same transport equations for $\mathrm{k}$ and epsilon as those employed for the $\mathrm{S}(k-\varepsilon) \mathrm{HR}$ are also used for the non-linear models. Moreover, the coefficient $c_{\mu}$ is no longer constant. Instead, $c_{\mu}$ becomes a function of the dimensionless mean strain rate $S k / \varepsilon$, where $S=\sqrt{2 S_{i j} S_{i j}}$. 
Realizable $(k-\varepsilon)$ High-Reynolds-number [26], Realizable $(k-\varepsilon)$ Two-Layer

A successful development on the $\mathrm{S}(k-\varepsilon) \mathrm{HR}$ model is the realizable $(k-\varepsilon)$ high-Reynolds-number model, $\mathrm{R}(k-\varepsilon) \mathrm{HR}$ model, that Shih et al [36] developed by modifying the transport equation for the specific dissipation rate $\varepsilon$, where the production and dissipation forms in the last two terms in Equation (10), are defined as $C_{\varepsilon 1} S \varepsilon-\frac{\varepsilon}{k+\sqrt{v \varepsilon}} C_{\varepsilon 2} \rho \varepsilon$ in which the coefficient $C_{\varepsilon 1}$ is sensitive to the dimensionless mean strain rate $S k / \varepsilon$. Also to increase the model flexibility, coefficient $c_{\mu}$ is suggested to be variable, related to flow and turbulence properties. Therefore specific mathematical constraints on the normal straining, which are consistent with the physical behaviour of turbulence, are satisfied. The transport equation of $k$ retains the form of equation (9) and the eddy-viscosity is defined by equation (6), with time scale $T=k / \varepsilon$. As this is a high-Reynold-number model, the wall function is used to account for the effects of the near-wall turbulence. This is the conventional log-low-based version.

Combination of the high-Re realizable k-epsilon and the two-layer approach gives the Realizable $(k-\varepsilon)$ Two-Layer $\mathrm{R}(k-\varepsilon)$ TL model. This is an approach pioneered by the UMIST group in the 1980s with publications such as Azzola et al. [38], Iacovides et al. [39] and Iacovides and Launder [40]. The turbulence in the near-wall region is modeled using simpler low-Reynolds number models such as the mixing length or the one-equation, which do not require the fine resolution of the near-wall regions that low-Re two-equation models do. The flow domain is divided into the main and the near-wall regions. In the near-wall regions the turbulent properties $\varepsilon$ and $\mu_{\mathrm{t}}$ are defined in terms of the normal wall distance, $\varepsilon=k^{3 / 2} / l_{\varepsilon}$ and $\mu_{t} / \mu=f\left(R e_{y}\right)$. The prescribed length scale, $l_{\varepsilon}$, is proportional to the wall-normal distance, $\mathrm{y}$, and in the one-equation model of Wolfstein [41], is damped across the viscous sub-layer using the local turbulent Reynolds number $R e_{y}=\sqrt{k} y / v$, and the turbulent kinetic energy, $\mathrm{k}$, is obtained through the solution of a transport equation similar to that used in the high-Re $k-\varepsilon$. The values of $\varepsilon$ given in the inner layer are merged smoothly, using the merging function suggested by Jongen [42], with those calculated from the outer layer by solving the transport equation of $(k-\varepsilon)$ model away from the wall. It is important to note that the transport equation for $k$ is applied in all regions.

\section{$(\mathbf{k}-\boldsymbol{\varepsilon})$ Lien Low-Reynolds-Number [25], $(\mathbf{k}-\boldsymbol{\varepsilon})$ Lien Low-Reynolds-Number Non linear Quadratic [25], $(\mathbf{k}-\boldsymbol{\varepsilon})$ Two-Layer Lien Non-linear Quadratic [25]}

The low-Reynolds-number model suggested by Lien et al [25],(k-ع)Lien Low-Reynolds-Number, has similarities to that of Launder and Sharma [34] but with additional damping functions. The dissipation rate transport equation employs equation (10) with changes to the production and destruction terms to $\left[C_{\varepsilon 1}\left(P_{k}+P_{n l}+P^{\prime}\right)-C_{\varepsilon 2} f_{2} \rho \varepsilon\right]$, where $P^{\prime}$ an additional production term is given as:

$$
P^{\prime}=f_{2}\left(P_{k}+2 \mu k / y^{2}\right) \exp \left(-0.00375 R e_{y}^{2}\right)
$$

$f_{2}$ is a damping function, given as [ $\left.1-0.3 \exp \left(-R e_{t}^{2}\right)\right], R e_{t}$ is turbulence Reynolds number defined as $k^{2} / \varepsilon v$.

Another damping function is employed in the eddy-viscosity expression, equation (6), as described below:

$$
f_{\mu}=1-\exp \left[-\left(C_{d 1} \sqrt{R e_{y}}+C_{d 2} R e_{y}+C_{d 3} R e_{y}^{2}\right)\right]
$$

As shown above, two damping function are utilized, one in the eddy-viscosity expression and other for the dissipation rate destruction and production terms. These damping functions are employed in order to take into account the effect of molecular viscosity near the wall, in which the turbulence is low. The damping function $f_{2}$ is employed in the expression for the additional production term $P^{\prime}$ to ensure that $P^{\prime}$ approaches zero at low turbulent levels. $P^{\prime}$ is introduced to ensure that the right level of near-wall turbulence energy dissipation is returned. As can be seen in the expression for $f_{\mu}$ above, in this models, in common with the practice followed by a number of low-Re models, the damping function explicitly includes the wall distance. This is generally undesirable since it makes the model non-local and in complex geometries the prescription of the wall distance becomes more approximate.

The same $k$ and $\varepsilon$ transport equations of the low-Re version of the linear eddy-viscosity, $k-\varepsilon$ of Lien [25] are also employed for those of the $k-\varepsilon$ Lien low-Reynolds-number non-linear quadratic model.

The $k-\varepsilon$ Two-Layer Lien non-linear quadratic model is a combination of $k-\varepsilon$ Lien high-Reynolds-number non-linear quadratic model and the two-layer approach. Therefore the transport equations are similar to those in the $k-\varepsilon$ Lien highReynolds-number non-linear quadratic model and also the equations of the two-layer approach are the same as those in the realizable $k-\varepsilon$ Two-Layer model.

SST $(\boldsymbol{k}-\boldsymbol{\omega})$

The $k-\omega$ model of Wilcox [43] which includes a transport equation for $k$ and one for the specific dissipation rate $\omega$. In external flows, the predictions of this model are influenced by the boundary value of $\omega$ in the free stream. To overcome this deficiency, Menter [27] suggested combining the $k-\omega$ and $k-\varepsilon$ models to create the shear stress transport $\operatorname{SST}(k-\omega)$ model. It is designed to remove the free-stream sensitivity by using a blending function based wall distance to 
switch from the $k-\omega$ at the near-wall regions to the $k-\varepsilon$ model away from the wall. The $k-\varepsilon$ transport equations, equations (9) \& (10), are organized through the relationship, $\varepsilon=\beta^{*} \omega k$, to follow a similar format to that of the $k-\omega$ equations of Wilcox. The version of the $\operatorname{SST}(k-\omega)$, employed in this study, is a low-Reynolds-number model because $\omega$ can be integrated up to the solid surface. Consequently a fine near-wall mesh is needed. A second blending function is employed in the model so as to switch to one of the two available eddy-viscosity approaches, either equation (6) or that originated by Johnson and King [44]. The latter can improve model predictions in regions of adverse pressure gradient and wake flows.

\section{$\mathbf{v}^{2}-\boldsymbol{f}(\boldsymbol{k}-\boldsymbol{\varepsilon})$}

The $\mathrm{v}^{2}-f(k-\varepsilon)$ Low-Reynolds-number model $[28,29]$ solves transport equations for $k$, $\varepsilon$, the parameter $\mathrm{v}^{2}$ which is used as a damping function for the turbulent viscosity, and can best be described as the normal turbulent stress perpendicular to the local flow streamline, and an elliptic relaxation function $(f)$. The $\mathrm{v}^{2}-f(k-\varepsilon)$ model uses equations (9) and (10) for $\mathrm{k}$ and epsilon respectively, but with the production term multiplied by $1+a \sqrt{k / \overline{\mathrm{v}^{2}}}$. The variable $f$ which appears in the transport equation for $\mathrm{v}^{2}$ is the solution to the elliptic relaxation equation. The elliptic function is employed to calculate a term analogous to the pressure-strain correlation of the Reynolds-stress model. The strength of the $\mathrm{v}^{2}-f$ model, is that it can account for the effects of the wall damping without the use of non-local parameters like the wall distance. Its weakness is the inclusion of extra differential equations and numerical stability problems related to the implementation of boundary conditions for $f$.

Although the non-linear EVMs can improve the model sensitivity to the anisotropy of normal Reynolds stresses, there are still some constraints that cannot be addressed without introducing to Reynolds stress models.

\subsubsection{Reynolds stress models}

The Reynolds stress models (RSM), which are also called second-moment closures (SMC), are the most complex turbulence models. In the previous models, the turbulent stresses tensor is directly related to the mean flow strain rate tensor. In Reynolds stress closures, as the name implies, the components of the Reynolds stress tensor are obtained through the solution of separate transport equations. Exact transport equations for the turbulent stresses can be derived, but these contain further unknown terms which need to be modelled. Reynolds stress models therefore are also approximate, but in contrast to the simpler models they can exactly account for the convection, diffusion and generation rate of the Reynolds stresses. The resulting turbulence field is consequently anisotropic, which in complex flows, see Iacovides et al [45] and Bo et al [46] can result in predictive improvements.

RSM models require more computational effort than the previous models. Some RSM models, like that of Gibson and Launder account for the wall influences on the Reynolds stresses anisotropy through the so called wall-reflection term employing low- or high-Reynolds-number approaches [47, 48, 31].

The starting point for the development of RSM is the exact differential transport equation for the Reynolds stresses $\rho \overline{u_{i} u_{j}}$ (equation (14)).

$$
\begin{array}{r}
\underbrace{\frac{\partial}{\partial t}\left(\rho \overline{u_{i} u_{j}}\right)}_{\begin{array}{c}
\text { Local Time } \\
\text { Derivative }
\end{array}}+\underbrace{\frac{\partial}{\partial x_{k}}\left(U_{k} \rho \overline{u_{i} u_{j}}\right)}_{\text {Convection, } C_{i j}}=\underbrace{\frac{\partial}{\partial x_{k}}\left[\mu \frac{\partial}{\partial x_{k}} \overline{u_{i} u_{j}}\right]}_{\text {Molecular Diffusion, } D_{L, i j}} \underbrace{-\frac{\partial}{\partial x_{k}}\left[\rho \overline{u_{i} u_{j} u_{k}}+\overline{p\left(\delta_{k j} u_{i}+\delta_{i k} u_{j}\right)}\right]}_{\text {Turbulent Diffusion, } D_{T, i j}} \underbrace{-\rho\left(\frac{\partial U_{i} u_{k}}{\partial x_{k}}+\overline{u_{j} u_{k}} \frac{\partial U_{i}}{\partial x_{k}}\right)}_{\text {Stress Production, } P_{i j}} \\
-\underbrace{2 \mu \frac{\partial u_{i}}{\partial x_{k}} \frac{\partial u_{j}}{\partial x_{k}}}_{\text {Dissipation, } \varepsilon_{i j}}+\underbrace{p\left(\frac{\partial u_{i}}{\partial x_{j}}+\frac{\partial u_{j}}{\partial x_{i}}\right)}_{\text {Pressure Strain, } \phi_{i j}}
\end{array}
$$

As commented earlier, in the resulting equations, the transient, convective transport $C_{i j}$, molecular diffusion $D_{L, i j}$, and stress production $P_{i j}$ require no modelling. The terms remaining to be modelled are the turbulent diffusion term $D_{T, i j}$, the dissipation term $\varepsilon_{i j}$ and, perhaps the greatest challenge, the pressure-strain term $\phi_{i j}$. The generalized gradient diffusion hypothesis of Daly and Harlow [49] is the most popular model for $D_{T, i j}$, though often for simplicity, which is also the case here, the isotropic model of the turbulent diffusion is adopted [50]:

$$
D_{T, i j}=\frac{\partial}{\partial x_{k}}\left(\frac{\mu_{t}}{\sigma_{k}} \frac{\partial \overline{u_{i} u_{j}}}{\partial x_{k}}\right)
$$

The eddy-viscosity is defined in equation (6), with time scale $T \equiv k / \varepsilon$, the turbulent kinetic energy defined as $k \equiv 0.5 \overline{u_{\iota} u_{J}}$ and the scalar turbulent dissipation rate $\varepsilon$ is computed from a transport equation analogous to the $k-\varepsilon$ model:

$$
\frac{\partial}{\partial t}(\rho \varepsilon)+\frac{\partial}{\partial x_{j}}\left(\rho U_{j} \varepsilon\right)=\frac{\varepsilon}{k}\left[C_{\varepsilon 1} P_{i i}-C_{\varepsilon 2} \rho \varepsilon\right]+\frac{\partial}{\partial x_{j}}\left[\left(\mu+\frac{\mu_{t}}{\sigma_{\varepsilon}}\right) \frac{\partial \varepsilon}{\partial x_{j}}\right]
$$


The above equations represent a base construction for the following two versions of the RSM used in this study.

\section{Gibson and Launder [30], RSM-GL}

Gibson and Launder [30] proposed a Reynolds stress model for high-Reynolds-number turbulent flows with a wall-function to deal with the near wall flows. Therefore the turbulent dissipation rate tensor in equation (16) is described as $\varepsilon_{i j}=$ $2 / 3 \rho \delta_{i j} \varepsilon$, accounting for isotropy of the small-scales [51].

One of the most challenging modelling tasks is how to account for the effects of the pressure-strain correlation $\phi_{i j}$. A linear approach is adopted in the RSM-GL. There are four distinct contributions to this process. Two of them isotropise the turbulence field, by redistributing the turbulence energy from the direction it is concentrated to the other two, at regions that there is either anisotropy in the turbulence energy itself, or in its rate of production. The other two terms are only active in the near-wall regions, where they take away fluctuating energy from the wall-normal direction and distribute it in the two wall-parallel directions. In the Gibson-Launder version, the wall distance and wall-normal direction appear explicitly in the latter group of redistribution terms, which are known as wall-reflection terms.

\section{Speziale, Sarkar and Gatski [31], RSM-SSG}

Speziale, Sarkar and Gatski [31] proposed an alternative version of the RSM in which the main difference with the G-L version is in the redistribution term. The isotropisation of production is now quadratic in terms of the strain rate tensor and one consequence is that there is no need to use wall-reflection terms to model the re-distributive effect of the walls on the turbulence structure.

As mentioned earlier, full details of the models are provided in the Appendix that follows the main text.

\section{Sliding Grids}

The experiments simulated in this study involve flow past a scaled down wind turbine placed in a large wind tunnel. The flow domain consequently involves the rotating blades of the wind turbine at the centre and stationary outer boundaries. Such flows can be best resolved through the use of a combination of rotating and stationary meshes. Within the structured grid approach there are two main options, shown in Figure 1, which can involve either an overlapping, Chimera mesh where a rotating cylindrical mesh is superimposed on a stationary Cartesian mesh, or the sliding mesh method, where a rotating cylindrical mesh at the centre slides past a non-overlapping outer stationary and usually non-orthogonal mesh. For the Chimera mesh, over most of the region covered by both meshes the flow equations are discretized and solved over the rotating mesh and at the interface there is an overlap region over which the flow equations are solved over both meshes. Interpolation is used to provide boundary condition to the rotating mesh from the numerical solution of the stationary mesh and vice-versa. While the transfer of information across the two meshes is more complex in the case of the Chimera mesh, than in sliding meshes, grid generation is simpler.

Blades and Marcum [52] recommended to employ sliding mesh approach for geometries which have known rotational motion. In the sliding mesh approach no hole cutting is necessary, making it computationally less expensive. Therefore the sliding mesh approach is widely employed in applications such as turbomachinery, stirred tanks and wind turbines. An apparent disadvantage of the sliding mesh is that the interface splits the flow field into two separate domains. The only way to make these separate domains aware of changes on the other side is by updating the values at the interface. Therefore it is very significant to update the values at the interface frequently throughout the time step.

A direct interface is employed to connect between the boundaries in the grid either permanently (for stationary cases) or temporarily for some time period (for moving cases). In STAR-CCM+ the direct interface includes several interface types and for this work the internal interface type is employed.

\section{Wind turbine rotor blade geometry construction, experimental data, and Computational Mesh}

The focus of this study is the simulation of the flow field around the rotor blade of NREL Phase VI HAWT [7]. The turbine consists of two blades with rotor 10.058m diameter as shown in Figure 2. The NREL S809 airfoil is employed as the blade cross section from the root to the tip with various chords and twist angles, producing tapered and twisted blade. The NREL Phase VI unsteady aerodynamics experiments $[7,53]$ were carried out using the NASA Ames large scale wind tunnel. The computations have been validated against a series of tests carried out with the blades rotating at constant rotational speed and over a range of wind speeds. At each test the experiments provide measurements of the local wall pressure around the blade surface at 5 radial locations, the circumferentially-averaged values of the normal force coefficient at the same radial locations and the global values of the power and thrust coefficients for each test. Since there are two independent velocity scales here, two independent dimensionless groups are needed to characterize each test. The two chosen here are the rotational Reynolds number $\left(R e_{\theta}\right)$ based on the rotational speed of the outer blade tip and the blade radius $\left(R e_{\theta}=\Omega \mathrm{R}^{2} / \nu\right.$, where $\Omega$ is the blade rotational speed, $\mathrm{R}$ is the rotor radius and $v$ is the air kinematic viscosity), and the tip-speed ratio 
(TSR), based on the ratio between the rotational speed of the outer blade tip and the free stream wind speed (TSR $=\Omega \mathrm{R} / U_{\infty}$, where $U_{\infty}$ is the free stream wind speed). The former is chosen because on the one hand it remains constant for all the tests considered but also because at these high values of the rotational Reynolds number the flow is expected to become independent of it. The latter dimensionless group is chosen because at this high rotational Reynolds numbers it is the one likely to exert the strongest influence on the flow. These issues will be further explored later in the paper.

The computational domain in Figure 3 replicates the NASA-Ames wind tunnel dimensions of $24.4 \mathrm{~m}$ height and $36.6 \mathrm{~m}$ width, with streamwise dimension of $170 \mathrm{~m}$. The rotor is placed $20 \mathrm{~m}$ downstream of the inlet. Two computational domains are generated, one for low-Reynolds-number LR models and other for high-Reynolds-number HR models. Each computational domain consists of two parts, the rotating cylinder and the stationary wind tunnel connecting with each other employing the sliding mesh technique. The entire computational domain consists of hexahedral meshes. An O-grid topology is employed for the flow domain and around the two blades. The blade grid is split into three parts; a blade, root, and tip part. The number of hexahedral cells for the blade and root parts in Figure 4 is respectively 97 and 29 in the spanwise orientation, and in the chordwise orientation 158 hexahedral cells for LR models and 142 for HR models covered both the upper and lower sides of the blade. The grid blocks of the rotor blade and the cylindrical part with a slice mesh through the rotor plane are demonstrated in Figure 5. Figure 6 displays the grid distribution around the blade tip, and close up view of mesh near both the leading- and trailing edges. The distance of the layer next to the wall is around $0.01 \mathrm{~mm}$ for LR models and $0.7 \mathrm{~mm}$ for HR models. The vertical spacing has an increase rate of 1.1 and includes 40 control volumes for LR and 20 for HR. The total number of control volumes in the computational domain is $6.65 \times 10^{6}$ for low-Reynolds-number models and $5 \times 10^{6}$ for high-Reynolds-number models.

The simulations have taken approximately 97 hours per LR case and 76 hours per HR case on the Computational Shared Facility (CSF) at the University of Manchester, using 32-core nodes, 64GB RAM, and 2.6 GHz power. One rotor cycle is accomplished within $0.8333 \mathrm{~s}$ using $0.001156 \mathrm{~s}$ time step or $0.5^{\circ}$ blade rotation. CFD computations have been performed for ten rotor cycles or $8.333 \mathrm{~s}$, providing 7209 sampling data, in order to ensure stable flow computations.

\section{Grid Sensitivity tests}

The grid dependence of the numerical solution has first been investigated, in order to arrive at a mesh size which can reduce numerical errors to an insignificant level. Seven hexahedral meshes sizes from 0.5 to 10 million cells have been employed to determine the mesh size beyond which, as shown in Figure 7(a), a further mesh refinement no longer changes the values of power and thrust predicted with a linear, low-Re $k-\varepsilon$ turbulence model, namely the $(k-\varepsilon)$ LLR. Figure 7(a) demonstrates that for the range of tip-speed ratio for which data are available and at a rotational Reynolds-number $R e_{\theta}=$ $1.31 \times 10^{7}$, the predicted values of these key performance parameters no longer change for mesh sizes larger than $6.65 \mathrm{M}$ cells. Further evidence on the grid independence of the solution is provided in the local pressure coefficient comparisons of Figure 7(b). These show that for the two tip-speed ratio extremes the distributions predicted by a mesh of $6.65 \mathrm{M}$ cells (using a low-Re model) and one of $10 \mathrm{M}$ are practically identical. Consequently the $6.65 \mathrm{M}$ cell mesh is employed for all computations which involve low-Reynolds-number models. The same grid is employed to create computational mesh for high-Reynolds-number models by reducing the nodes close to the blade surface to just 20 nodes and the whole grid to $5 \times 10^{6}$ cells.

The $\mathrm{y}^{*}$ values of the near-wall nodes over the blade upper and lower surfaces, for the grids chosen for low-Reynoldsnumber and high-Reynolds-number models are presented in Figures 8(a) and 8(b) respectively. As can be seen, use of the low-Reynolds-number mesh results in near-wall-node $\mathrm{y}^{*}$ values of order 1 or less over the entire blade surface, while for the high-Reynolds number grid, the corresponding $\mathrm{y}^{*}$ values range from 30 to 75 over most of the blade surface.

\section{Results and discussion}

\subsection{Power Coefficient and Normalised Power}

The value of the power coefficient at each set of conditions, is obtained through the numerical integration of the surface pressures over the entire blade surface which provides the torque around the axis, which is then multiplied by the angular velocity to result in the power. For the power coefficient, the power is non-dimensionalised by $1 / 2 \rho_{\infty} U_{\infty}^{3} \pi R^{2}$, and for the normalised power by $1 / 2 \rho_{\infty}(\Omega R)^{3} \pi R^{2}$ where $\rho_{\infty}$ and $U_{\infty}$ denote the free-stream values of the air density and wind speed respectively, and $\Omega$ is the angular velocity of the rotor blade. Figures $9 \mathrm{a} \& 9 \mathrm{~b}$ assess the effectiveness of the models tested in this investigation in reproducing the variation of the power coefficient and the normalised power with the inverse of the tip speed ratio, which can be viewed as a dimensionless wind speed. For clarity the comparisons are organized in two parts; Figures 9a(i) and 9b(i) present comparisons which involve the simpler, linear effective viscosity models and Figure 9a(ii) and $9 \mathrm{~b}$ (ii) the corresponding comparisons for the more advanced, non-linear effective viscosity and Reynolds stress transport models. The power coefficient is essentially the ratio between the aerodynamic power produced by the wind turbine and the rate at which kinetic energy is transported by the wind. As indicated by the experimental data in Figure 9a, at low wind speeds relative to the blade tip speed (TSR 5 of greater), the power coefficient has a value of just under 0.4 . As 
the wind speed increases, the angle of attack (the angle between the resultant wind speed relative to the rotating blade and the blade itself) increases, which will eventually lead to stall. As a result at values of TSR less than 5 there is initially a rapid reduction in the power coefficient with a point of inflection at around TSR of 3 and then for values lower than that the power coefficient approaches zero asymptotically as 1/TSR increases further. All turbulence tested return this overall variation of power coefficient with the inverse of the tip speed ratio, but in the case of the simpler linear effective-viscosity models, as can be seen in Figure 9a(i), the deviation between predictions and experimental data is wider than for the more refined non-linear and stress transport models, summarised in $9 \mathrm{a}$ (ii). While at first glance the deviation between the predicted and measured values of the power coefficient look small, these occur in regions where the absolute value of the power coefficient is low and are thus indicative of far larger deviations between the predicted and measured actual power. It is for this reason that the comparisons between measured and predicted power normalised by the tip speed $\Omega R$, which is independent of the wind speed, have been included in Figure 9b. The experimental data in Figure 9b show that initially the power rises sharply and linearly with the inverse of the tip-speed ratio, but subsequently reaches a local maximum at about $\mathrm{TSR}=4$, followed by a gentle reduction with further decrease in tip-speed ratio until the value of 2.2 , where there is a local minimum. A moderate steady increase in the normalized power takes place when the tip-speed ratio is lower than 2.2. This non-linear and non-monotonic variation of power with the inverse of the tip-speed ratio suggests that the flow regime around the rotating blade undergoes fundamental changes as the as the value of the tip-speed ratio is changed. The first question being addressed with these comparisons is whether the turbulence models under investigation can reproduce these flow changes. Even a casual look at Figures 9(a) and 9(b) is sufficient to make the point that, in general, stress transport and non-linear effective-viscosity models provide significantly more reliable predictions than the linear effective-viscosity approaches. All models appear to predict correctly the initial steep rise of the normalized power with the inverse of the tipspeed ratio, presumably because over this range the flow around the rotating blade is simpler. Beyond this initial region, where the flow features probably become more complex, the predictions of a number of these models start to deviate from the measured behaviour. Most notable is how substantially the normalized power is under-estimated by linear EVM models widely used in industry, mainly through commercial codes, such as the SST and the $\mathrm{v}^{2}-f$. Indeed, of the linear EVM models tested only the high-Reynolds number version of the realizable $k-\varepsilon$ and the low-Re version of the Lien et al $k-\varepsilon$ can be considered reliable. By contrast, the comparisons of Figure 9b(ii) demonstrate that the deviation among the predictions of the different stress transport and non-linear $k-\varepsilon$ models is far narrower. The Gibson-Launder version of the stress transport model and the two high Reynolds-number versions of the non-linear $k-\varepsilon$ models (quadratic and cubic in terms of the strain rate tensor) predict values of the normalized power which are in very close agreement with the measured ones, over the entire range of tip-speed ratios. Perhaps the most surprising outcome of the comparisons of Figure $9 \mathrm{~b}$ (ii) is that the predictions of the SSG version of the stress transport model are not as close to the measurements as those of the three above-mentioned models. The reasons for the predictive successes and failures of these models are discussed in further detail, later in this paper when comparisons of local flow parameters are presented.

\subsection{Normal Force Coefficient}

Comparisons of the radial variation of the circumferentially averaged normal force coefficient, in a sense a semi-global parameter, shown in Figure 10, provide a further assessment of the models' capabilities. These comparisons are presented over the full range of tip-speed ratio for which data are available, listed in Tables 2. While the normalized power and power coefficient, discussed in the previous section depend on the component of the pressure force acting on the blade surface along the circumferential direction, the normal force coefficient depends on the corresponding component of this force in the axial (wind speed) direction. The experimental data show that at the highest tip-speed ratio, the normal force coefficient is relatively low and uniform over the radius of the blade. As the tip-speed ratio decreases, the normal force coefficient first rises uniformly over the blade radius and subsequently continues to rise, but displays a non-uniform radial distribution with the lowest level at the outer tip of the blade and a monotonic and substantial rise along the blade in the inward direction. The ratio between the innermost and outermost values becomes greater than 2:1. As was also the case with the power variation with tip-speed ratio, here again there is an indication that the flow structures around the rotating blade change as the tip-speed ratio decreases.

At the higher tip-speed ratios all the turbulence models return very similar predictions of the radial distribution of the normal force coefficient, which are in close agreement with the data. This is consistent with the corresponding power comparisons of Figure 9 and provide further support for the hypothesis that at the higher tip-speed ratios the flow field around the rotating blades is relatively simple. As the tip-speed ratio decreases the predicted radial variations of the force coefficient start to deviate from each other, with these deviations becoming strongest and substantial at the intermediate tip-speed ratios. These deviations diminish at the lowest tip-speed ratios, but do not entirely disappear. The fact that the predictions of a number of turbulence models become poor at the intermediate tip-speed ratios suggests that it is failure to correctly predict the changes from the high to the low tip-speed ratio flow fields which leads to poor predictions of the normal force coefficient. The models returning the poorer predictions include linear eddy viscosity models like the SST $k-\omega$, the $\mathrm{v}^{2}-f$, the two versions (high-Re and low-Re) of the realizable $k-\varepsilon$ and the high-Re version of the quadratic non-linear $k-\varepsilon$. The predictions of the two versions of the stress transport model and of the cubic non-linear $k-\varepsilon$ are in close agreement with the measurements. Once again the greater reliability of the more refined models is demonstrated. The fact that for the normal force coefficient predictions are generally closer to the data than for the power suggests that the pressure force in 
the axial direction, mainly caused by the wind impinging on the flat surface of the blade, is less sensitive to the turbulence field.

\subsection{Surface Pressure Coefficient}

The comparisons of the variation of the local surface pressure coefficient around the blade, shown in Figures 11 to 13, for a number of radial locations, provides a more detailed insight to the flow behaviour. At the higher tip-speed ratios, the measurements show that there is little change in pressure across the blade and what there is, is confined to the leading edge of the rotating blade. There is also very little variation in the radial direction. As the tip-speed ratio decreases, first the difference between the pressure across the blade starts to rise and subsequently, over the inner region, the pressure difference across the blade starts becoming more uniform in the circumferential direction. These observations are consistent with the observed variations in the power and normal force coefficients of Figure 9a and 10 respectively. As far as comparisons with the current computations are concerned, notable deviations between predicted and measured distributions start to appear at intermediate wind speeds near the leading edge of the blade and over the upper surface. Initially these deviations appear in the SST $k-\omega$ model, the $v^{2}-f$, and the low-Reynolds-number version of the quadratic non-linear model and then spread to the two-layer form of the quadratic non-linear model and also to the two-layer form of the Realizable $k-\varepsilon$ model and the two high-Reynolds-versions of the quadratic and cubic non-linear models. The predictions of the SSG version of the stress transport model also show some discrepancies in comparison with the experimental data, at this intermediate wind speed region. At the lower tip-speed ratios, as also noted in earlier comparisons, differences between predictions and measurements start to diminish, though for models such as the $\mathrm{v}^{2}-f$, the high-Re $k-\varepsilon$ and the Lien et al low-Re $k-\varepsilon$ their predictions still show differences with the measured values of the pressure coefficient, over the inner region, which extend from the leading to the trailing edge of the upper surface. As expected, the models that exhibited predictive deficiencies in the comparisons of the global parameters are the ones that also fail to reproduce some of the features of the variation of the local pressure coefficient.

\subsection{Instantaneous Flow Field}

In order to further understand the flow characteristics involved and also the reasons for the models' predictive characteristics; Figures 14 to 19 present selected predictions of instantaneous flow path-lines over the upper surface of the blade and also flow path-lines and static pressure contours within planes normal to the blade surface, at five radial locations.

At the highest tip-speed ratio, the predictions of the three turbulent models depicted in Figure 14 are also typical of all the others. The flow remains attached over the upper blade surface (the surface on the downwind side of the blade) and the pressure is higher on the lower surface, the surface which acts as the impingement surface for the oncoming wind. This pressure difference of course generates a force normal to the blade surface, which leads to the drag force on the wind turbine and also, due to the blade angle of attack, a force in the circumferential direction, towards the left in Figures 14 to 19, which leads to the blade rotation and power generation.

As the tip-speed ratio decreases, Figure 15 shows that the flow field over the upper (downwind) surface of the blade becomes more complex and the predictions of different turbulence models start to vary. A region of either slow-moving or re-circulating, relative to the rotating blade, flow is formed near the trailing edge of the upper surface with a strong radial velocity component in the outward direction. This region at the inner part of the blade extends over most of the upper surface, but its size gradually diminishes further outwards until it disappears. The stronger wind-speed generates a region of very low pressure over the upper part of the leading edge. As a result there is a strong adverse pressure gradient over the upper surface, which causes the flow to separate towards the trailing edge. Because in absolute terms, the fluid within the re-circulation bubble has a rotational speed similar to that of the rotating blade, the centrifugal force drives it radially outwards. Since the flow field is now far more complex, it is reasonable to expect the predictions to become sensitive to the turbulence model used. This is confirmed by the plots of Figure 15, which show that the radial extend of the recirculation bubble and the pressure over the upper surface predicted by the different models vary substantially. The SST and the SSG predict that the separation bubble extends to $90 \%$ of the blade radius, while with the high-Re $k-\varepsilon$ the predicted length is down to $60 \%$. The Gibson-Launder version of the RSM, which is the model in closest agreement with the experimental data, predicts that the separation region extends to around $80 \%$ of the blade radius.

As can be seen in Figures 16 to 19, as the tip-speed ratio decreases further, the recirculation bubble expands both in the radial and the circumferential directions until, as shown in Figure 18, for a tip-speed ratio of 2.5, the recirculation region extends over the entire upper surface of the blade. The differences in the predictions of the turbulence models tested appear to be strongest at the intermediate tip-speed ratios, at which the upper surface is only partially covered by the recirculation bubble. When the flow over the upper surface of the blade is either fully attached or fully separated the different models return similar flow predictions. 


\subsection{Mean Local Velocity Profiles}

Another view of the flow development around the rotating blade is provided through the mean velocity profiles of figures 21 to 23, predicted using the Gibson-Launder stress transport model. These are profiles of the circumferential, radial and axial components of the velocity field, relative to the rotating blade, along traverse lines within planes normal to the blade surface, at three different radial locations, shown in Figure 20.

Starting from the highest tip-speed ratio case, shown in Figure 21, the circumferential velocity profiles (the three top sets of profiles) show that the circumferential flow remains attached over both the upper and lower surface. As also noted earlier the velocity field is relatively uncomplicated at this low wind speed. The axial velocity profiles, for the innermost location show that over the lower side the axial flow moves towards the blade surface, while over the upper side the fluid moves away from the blade surface. At the mid-blade and outer locations, because of the stronger curvature of the blade surface the axial flow direction is reversed over the lower surface near the leading edge and over the upper surface near the trailing edge. The radial velocity profiles show that for the inner and mid-blade planes the fluid is pumped outwards by the centrifugal force and along the lower surface the radial motion is very weak. At the outer plane, the radial motion conditions are reversed. The path-line plots of Figure 14 suggest that this is due to the presence of the trailing edge vortex.

At the lower tip-speed ratios for which the corresponding profiles are presented in Figures 22 and 23. The most notable difference is the reversal of the circumferential velocity over the upper surface. As a result, the thickness of the circumferential boundary layers over the upper surface of the blade is progressively increased, especially at the inner and mid-plane locations. This in turn leads to a large region of low axial velocity over the upper surface and strong radially outward motion. These profile plots confirm the complexity of the flow over the upper (downwind) side of the rotating blades at the higher wind speeds.

\subsection{Turbulence Shear Stress Profiles}

Figures 24 and 25 present profiles of the two components of the turbulent shear stress which include the velocity fluctuations in the axial direction, at the same locations around the blade surface, for which mean velocity profiles have been presented. These two components exert the strongest influence on the mean flow development around the blade and also on the generation of turbulence. These profiles have been predicted by a number of different turbulence models. They are only presented for a tip-speed ratio of 2.9 and $R e_{\theta}=1.31 \times 10^{7}$, which is one of the intermediate wind speeds at which the upper surface of the blade is partially covered by the separation bubble. Since these are time-dependent flow simulations there are two contributions to the Reynolds stresses. One is the modeled contribution of the smaller scales of turbulence and is obtained from either the solution of transport equations for the turbulent stresses, or the effective viscosity approximation and the other is the resolved contribution of the large scales and it is obtained from the post-processing of the instantaneous flow field. Figure 24 and 25 present the total (continuous lines) and resolved components (broken lines).

Starting with the profiles predicted by the Gibson-Launder version of the RSM model, which is in closest agreement with the data, the profiles of both components show that the turbulent shear stress is negligible over the lower side of the blade, where the circumferential flow is largely undisturbed and there is practically no radial motion. Over the upper side where the circumferential boundary layer separates and there is a strong radial motion, both components show a significant variations which are indicative of the presence of strong turbulent structures. The turbulence levels are especially high at the mid-blade $(\mathrm{r} / \mathrm{R}=63 \%)$ location, where these turbulent structures extend about a chord length away from the upper surface, in the downwind direction and which is consistent with the corresponding mean flow profiles It is also noticeable that around the leading edge at the inner plane $(\mathrm{r} / \mathrm{R}=30 \%)$ and around the trailing edge of the outer location $(\mathrm{r} / \mathrm{R}=95 \%)$ the resolved components make a sizeable contribution to the total shear stress, which indicates the presence of resolved, large-scale unsteady structures.

The two $k-\varepsilon$ models, the high-Re and the Lien et al. [25] low-Re versions, return similar shear stress predictions. The stress levels are generally higher than those of the GL-RSM and in contrast to those of the RSM, the effective-viscosity profiles do not change sign along a traverse line. These differences arise from the insensitivity of the effective viscosity approximation to the effects of streamline curvature and strong shear as demonstrated in Choi et al [54] and Klein et al [23]. Another most notable difference between RSM and $k-\varepsilon$ predictions is the strong spike in the circumferential-axial shear stress at the leading edge of the blade. This is due to the well known predictive flaw of the effective viscosity models at the stagnation point region, explained by, among others, Craft et al [55]. The effective-viscosity models fail to return significant levels of the resolved components, certainly a lot lower than what the Gibson-Launder RSM does, which in turn suggests that they fail to resolve large-scale unsteady structures around the blade. Possibly as a result of this, at the outer location $(r / R=95 \%)$ the $k-\varepsilon$ models predict a thinner region of significant turbulence levels over the upper surface.

The predictions of the SST model also differ from those of the Gibson-Launder RSM. The shear stress profiles with the circumferential-axial fluctuations predicted by the SST suggest that at the inner and outer planes $(\mathrm{r} / \mathrm{R}=30 \%$ and $95 \%)$ these fluctuations are confined over narrower regions above the upper blade surface, The SST also predicts mostly zero resolved components, which suggest that like the other EVM models, the SST also predicts weaker three dimensional unsteady structures. Moreover, at the outer location $(\mathrm{r} / \mathrm{R}=95 \%)$ the SST model produces a spike at the stagnation point region. The 
shear stress profiles with the radial-axial fluctuations predicted by the SST, on the other hand are stronger than those of the $\mathrm{RSM}$ at the inner location $(\mathrm{r} / \mathrm{R}=30 \%)$, and weaker at the other two.

The predictions of the $v^{2}-f$ model also have their own individual features. The profiles of the circumferential-axial fluctuations returned by the $\mathrm{v}^{2}-f$, show that at the inner and mid-plane sections $(\mathrm{r} / \mathrm{R}=30 \%$ and $63 \%)$ the $\mathrm{v}^{2}-f$ model returns higher shear stress levels than the RSM at $\mathrm{r} / \mathrm{R}=30 \%$ and $60 \%$, but at the outer location the $\mathrm{v}^{2}-f$ predicted turbulent shear stresses are confined to a narrower region above the upper surface of the blade. The corresponding comparisons of the radial-axial shear stress present a similar picture.

The above comparisons show that in the presence of flow impingement, streamline curvature, strong shear and flow separation, the different categories of models respond very differently, which consequently leads to the differences in the resulting flow field predictions. The response of the Reynolds stress closure of Gibson and Launder [30] appears to be the one that most closely resembles that of the real flow.

\subsection{Vortex structure}

The structure of the flow departing the wind turbine blades is studied by visualising the evolution of the iso-surfaces of Qcriteria, $Q=(1 / 2)\left(\|\Omega\|^{2}-\|S\|^{2}\right)$ with the value of $Q=5$, downstream of the rotating blades. Because the meshes described in Section 5 were only intended to accurately resolve the flow around the blades and in the region immediately downstream, for this task additional computations have been carried out with the Gibson-Launder stress transport model, using a mesh of $25 \mathrm{M}$ control volumes which maintains fine grid resolution for three rotor diameters downstream of the blades. Positive $\mathrm{Q}$ defines the flow regions having strong swirling or excess rotation rate in relation to the strain. This is illustrated in Figure 26 where the iso-surfaces computed by the Gibson-Launder RSM at different tip-speed ratios are presented. At the highest tip-speed ratio, where as seen in Figures 14 and 21 the flow around the blade remains attached, the blade rotation causes relatively little disturbance. The one exception to this are the trailing edge vortices, which due to the blade rotation and the convectively effect of the wind follow a helical path, and persist over the entire length of the monitoring domain. The vortical structures shed from the trailing edge of the rest of the blade are fairly weak, though they increase in strength somewhat near the inner base of the blade where according to the path line plots of Figure 14, there is a corner region of flow separation.

At the intermediate speed ratios the strength of the vortical structures shed from the trailing edge of the blade is increased. This is due to the separation region formed over the upper (downwind) side of the blade, observed in Figures 15 and 16. The trailing edge vortices are also present, but, due to the stronger wind speed, the helical angle increases.

At the lower tip-speed ratios the vortical structures shed from the trailing edge of the blade further increase in strength, which is consistent with the corresponding increase in the strength of the re-circulation region over the downwind surface of the blade, but the trailing edge vortices now disappear. The path-line plots of Figure 19, show that at the lower tip-speed ratios, the re-circulation region over the downwind surface of the blade extends over the entire blade radius. As also shown in Figure 19, there is a strong radially outward motion along the entire downwind surface of the blade, which suppresses the formation of trailing edge vortices. Each of the two rotating blades consequently generates a vortex structures within a plane coming off the trailing edge of each blade, which, due to the blade rotation and the transport effect of the wind, develops a helical shape. The resulting effect is therefore that of a double helix of vortex structures, reminiscent of the DNA structure.

The complex vortical structure and the attached and separated flow can be seen in the animations of Electronic Annexes AN1P, AN1S, AN2P, AN2S, AN3P, AN3S, and AN4P, AN4S. These animations show 3D rotation of the wind turbine rotor from the pressure side view (AN1P, AN2P, AN3P, and AN4P) and also from the suction side view (AN1S, AN2S, AN3S, and AN4S) applying RSM-GL model at rotational Reynolds-number $1.31 \times 10^{7}$ and tip-speed ratios 7.6, 3.5, 2.5, and 1.5.

\subsection{Time-dependent Pressure Coefficient and Spectral Analysis}

In Figure 27 we take a look at the ability of some of the unsteady RANS models tested to predict the unsteadiness of the measured pressure field. This comparison relates to a single point in the flow and a single flow case, so perhaps the comparisons between the total and resolved shear stress profiles in Figures 24 and 25 provides a more comprehensive evaluation of the models abilities to predict unsteadiness. Nevertheless, these limited comparisons between the measured and predicted time histories of wall pressure and their corresponding spectral distributions allow for a more complete assessment of the models' capabilities. More specifically, Figure 27 presents time history comparisons of the wall static pressure at a point on the trailing edge of the blade, at $\mathrm{r} / \mathrm{R}=0.3$ and for an tip-speed ratio of 1.5 and $R e_{\theta}=1.31 \times 10^{7}$ and also of the corresponding spectral analysis of the fluctuations, using the Fast Fourier Transform (FFT).

Starting with the experimental data, the instantaneous pressure, on the left hand of Figure 27, shows rapid changes superimposed on longer time-scale changes, which suggests that pressure fluctuations occur over a range of frequencies. The spectral distribution that results from the Fourier transform of the instantaneous pressure measurements do confirm the 
previous conclusion, showing a dominant peak in pressure fluctuations at a frequency about $3 \mathrm{~Hz}$ and then a series of lower peaks at frequencies extending beyond $30 \mathrm{~Hz}$. In terms of the dimensionless Strouhal number $(\mathrm{St}=\mathrm{fD} / \mathrm{U})$ the frequency of $3 \mathrm{~Hz}$ corresponds to St of 1.2 . In the predicted time histories of the wall pressure, all models return unsteady behaviour, but there are marked differences in the temporal variation in pressure produced by the turbulence models. The two $k-\varepsilon$ models return almost sinusoidal temporal variations, which lead to a spectral distribution with only a single peak, located at about the same frequency as the dominant frequency in the experimental data, but not as high. The Gibson-Launder version of the RSM produces a wall pressure time history similar to that produced by the $k-\varepsilon$ models, but with a higher peak at around $3 \mathrm{~Hz}$. This suggests that at this point and wind speed the GL-RSM is only able to reproduce the dominant flow fluctuation frequency. The time history predicted by the $\mathrm{v}^{2}-f$, on the other hand, shows very strong low frequency fluctuations. As a result, the spectral distribution shows a number of erroneously high peaks for frequencies up to $12 \mathrm{~Hz}$ and practically no fluctuations beyond that. By contrast the SST model predicts a spectral distribution similar to that of the experiments for frequencies up to $20 \mathrm{~Hz}$. This improved performance of the SST in comparison to the linear $k-\varepsilon$ models, is probably due to the feature in this model that limits the magnitude of the turbulent viscosity in regions of strong shear. The lower turbulent viscosity in such regions makes it more likely for the predicted flow to develop instabilities. This improvement in the SST predictions is in contrast to the generally poor performance of this model when it comes to key performance parameters like the normalized power. It is perhaps an indication that single point comparisons are of limited usefulness. The two non-linear models also manage to produce spectral distributions for frequencies up to $20 \mathrm{~Hz}$, reasonably close to those of the experimental data. In common with the SST model, in the non-linear models the turbulent viscosity is also reduced at higher strain rates. It is this common feature that enables both types of models to resolve a greater proportion of the pressure fluctuation spectrum. Finally the SSG version of the RSM, in contrast to the GL version over-estimates the fluctuation levels at the dominant frequency $(3 \mathrm{~Hz})$, but, more in common with the GL version, it fails to predict significant fluctuation levels at higher frequencies.

It thus appears that the models which directly allow for strain rate to reduce the turbulent viscosity, resolve a greater proportion of the fluctuation spectrum, extending to higher frequencies. This feature alone, however, is not sufficient for the satisfactory prediction of the entire flow field.

\subsection{Relevant Dimensionless Parameters}

As mentioned earlier, the two dimensionless groups selected here to fully describe each case are the rotational Reynolds number and the tip-speed ratios of the blade. One of the reasons for making this selection is the expectation/hypothesis that at the level of rotational Reynolds number considered here, the flow characteristics and the resulting dimensionless flow parameters are primarily dependent on the tip-speed ratio. To explore this, a further set of simulations has been produced, using the Gibson-Launder RSM, at a rotational Reynolds number of $2.62 \times 10^{7}$, which is twice as high as the one at which all the previous computations have been produced, and at tip-speed ratio values of 7.6, 2.9 and 1.5. The resulting comparisons between the predictions at $R e_{\theta}=2.62 \times 10^{7}$ with those at the corresponding tip-speed ratios at $R e_{\theta}=1.31 \times$ $10^{7}$, are presented in Figures 28 and 29.

Figure 28, compares the values of the two global performance parameters, namely the power coefficient and the normalised thrust at the tip-speed ratio values of 7.6, 2.9 and 1.5. These comparisons demonstrate that both these coefficients, at the same inverse tip-speed ratios, have practically identical values at the two different rotational Reynolds numbers.

Figure 29, on the other hand, focuses on the tip-speed ratio of 2.9 and compares the local flow fields around the blade, at the two rotational Reynolds numbers. While there are some minor differences in the local flow field around the blade, the main flow characteristics are essentially the same, namely a bubble of circumferential flow re-circulation over most of the upper, downwind, surface of the blade, apart from a short region near the outer blade tip, with also a strong outward radial motion.

These comparisons confirm that the selection of these dimensionless groups is indeed the most appropriate and that at high rotational Reynolds numbers, the tip-speed ratio, which is effectively the angle between the rotating blade and the resultant velocity relative to it, exerts the primary influence on the flow characteristics and the values of the flow dimensionless parameters.

\section{Conclusions}

CFD simulations of flow around the NREL Phase VI rotor are performed employing the 3D, unsteady, parallel, finite volume flow solver, STAR CCM+. The computations are conducted for the rotor blades in an inertial frame of reference using sliding mesh technique. The study has covered a wide range of tip-speed ratios between 7.6 and 1.5 (wind speeds 5 $25 \mathrm{~m} / \mathrm{s}$ ) and rotational Reynolds-number $1.31 \times 10^{7}$ at $3^{\circ}$ pitch angle and $0^{\circ}$ yaw angle, using twelve turbulence models.

The main contribution of this study is undoubtedly in identifying which of the turbulence models currently available in widely used CFD codes, are able to provide quantitatively reliable simulations of wind-turbine flows, using the costeffective unsteady RANS approach. Moreover, interrogation of the resulting predictions is used to advance our understanding of the flow structures present, which in turn helps to explain differences in the models' predictive 
performances.

When the ratio between the wind speed and the blade tip speed is low, the aerodynamic power increases linearly with wind speed (at a fixed rotational speed) and all models predict reliably key performance parameters, like the normalized power and the power and thrust coefficients. At higher ratios between the wind speed and the blade speed, where the relationship between the aerodynamic power and the wind speed (for a fixed blade rotational speed) becomes non-linear, the predictions that result from the use of different turbulence models start to deviate. As far as the predictions of the normalized aerodynamic power are concerned, these deviations in predictions are far wider in the case of the simpler linear effectiveviscosity models. Popular models of this type, like the SST $(k-\omega)$ and the $\mathrm{v}^{2}-f$, return some of the poorest predictions, under-estimating the normalized power by as much as $50 \%$ in places. The non-linear effective-viscosity and Reynolds stress transport models show narrower predictive deviations, making them a safer choice. In particular, the Gibson-Launder version of the Reynolds stress model, a high-Reynolds number model, and also the two high-Re versions of the non-linear effective viscosity model (quadratic and cubic) are the most reliable. Among the linear effective-viscosity models tested, the Lien et al low-Re $k-\varepsilon$ and the high-Re version of the realisable $k-\varepsilon$, though not as faithful as the three more refined models identified above, also produce reliable predictions. More extensive comparisons of profiles of the local pressure coefficient also confirm the above conclusions on the suitability of the turbulence models tested.

Examination of the predicted flow fields shows that at low wind speeds, relative to the blade tip speed, the circumferential flow remains attached over both sides of the rotating blade. And a circumferential vortex is formed at the blade tip, similar to the wing tip vortex of aircraft wings. It is because of the uncomplicated nature of the flow field over this range that all turbulence models tested have been able to produce reliable predictions. At higher wind speed to blade tip speed ratios, a flow re-circulation (in the circumferential direction) bubble is formed over the downwind surface of the blade, initially confined to the innermost location and the trailing edge region. As the wind speed increases further, the separation bubble expands in both the radial and circumferential directions to cover the entire downwind surface of the blade. Because in absolute terms the fluid within the separation bubble rotates with the same angular velocity as the blade, the centrifugal force drive the fluid within the separation bubble outwards. When the separation bubble extends over the entire blade surface, this outward motion suppresses the formation of the tip vortex. The flow field is evidently more complex in this operating range, with strong shear, streamline curvature, body force and impingement effects. Consequently, most turbulence models, especially those relying on the linear effective viscosity approximation, are unable to return the correct flow development at this operating range.

Limited investigation of the time histories of the wall pressure measurements has shown that the pressure fluctuations have a dominant peak at a frequency of $3 \mathrm{~Hz}$, which corresponds to a Strouhal number of 1.2 and smaller peaks at higher frequencies, up to $30 \mathrm{~Hz}$. All the RANS models used to simulate these flows as time-dependent are able to predict the dominant, low-frequency peak in pressure fluctuations but only non-linear effective viscosity models and the SST model, in which the turbulent viscosity is reduced in regions of strong shear, return some of the higher frequency peaks.

A limited parametric study, suggests that at the range of rotational Reynolds number for which the NERL VI experiments have been carried out, the flow characteristics, and the values of the dimensionless performance parameters are primarily determined by the value of the tip speed ratio.

It has thus been demonstrated that, provided that the right advanced turbulence models are used, the cost-effective method of unsteady RANS can be used to provide quantitatively reliable predictions of the flow around the wind turbine blades, in the downstream wake, and also the dominant frequency of the pressure fluctuations, all of which are critical to the development of more efficient and durable designs.

\section{Acknowledgments}

This research is funded by the Ministry of Higher Education and Scientific Research of Iraq. The reliable experimental data for NREL Phase VI HAWT rotor blade have been kindly provided by Dr Scott Schreck, from the NREL near Boulder, Colorado. His assistance is highly appreciated. The help provided by IT services at the University of Manchester for using their High Performance Computing (HPC) on the Computational Shared Facility (CSF) is deeply appreciated. 


\section{Appendix A. Description of turbulence models}

For the turbulence models employed in this study, the transport equations and other relationships with their constants are given in this appendix.

\section{A.1. $S(k-\varepsilon) H R,(k-\varepsilon) L H R N Q$, and $(k-\varepsilon) L H R N C$ model}

The transport equations for $k$ and $\varepsilon$ for these three models are given in equations (9) \& (10) respectively. The Reynolds stresses for $\mathrm{S}(k-\varepsilon) \mathrm{HR},(k-\varepsilon)$ LHRNQ \& $(k-\varepsilon)$ LHRNC models are computed by equation $(5),(7) \&(8)$ respectively. The eddy viscosity for the three models is defined by equation (6).

The coefficients for $\mathrm{S}(k-\varepsilon) \mathrm{HR}$ are:

$C_{\varepsilon 1}=1.44, C_{\varepsilon 2}=1.92, C_{\mu}=0.09, \sigma_{k}=1.0, \sigma_{\varepsilon}=1.3, C_{t}=1.0$

The same coefficients except $\mathrm{C}_{\mu}$ are used for $(k-\varepsilon)$ LHRNQ $\&(k-\varepsilon)$ LHRNC models.

and $\mathrm{C}_{\mu}$ now becomes:

$$
C_{\mu}=C_{a 0} /\left(C_{a 1}+C_{a 2} k / \varepsilon \sqrt{S_{i j} S_{i j}}+C_{a 3} k / \varepsilon \sqrt{\Omega_{i j} \Omega_{i j}}\right)
$$

The remaining coefficients for $(k-\varepsilon)$ LHRNQ $\&(k-\varepsilon)$ LHRNC models are:

$$
\begin{aligned}
& C_{1}=0.75 /\left(1000+\left(k / \varepsilon \sqrt{S_{i j} S_{i j}}\right)^{3}\right) C_{\mu}, C_{2}=3.75 /\left(1000+\left(k / \varepsilon \sqrt{S_{i j} S_{i j}}\right)^{3}\right) C_{\mu}, \\
& C_{3}=4.75 /\left(1000+\left(k / \varepsilon \sqrt{S_{i j} S_{i j}}\right)^{3}\right) C_{\mu}, C_{4}=-10 C_{\mu}^{2}, C_{5}=-2 C_{\mu}^{2}
\end{aligned}
$$

\section{A.2. $R(k-\varepsilon) H R \& R(k-\varepsilon) T L$ models}

The transport equation for $k$ is presented in equation (9) and for $\varepsilon$ is defined as:

$$
\frac{\partial(\rho \varepsilon)}{\partial t}+\frac{\partial}{\partial x_{j}}\left(\rho U_{j} \varepsilon\right)=\frac{\partial}{\partial x_{j}}\left[\left(\mu+\frac{\mu_{t}}{\sigma_{\varepsilon}}\right) \frac{\partial \varepsilon}{\partial x_{j}}\right]+C_{\varepsilon 1} S \varepsilon-\frac{\varepsilon}{k+\sqrt{v \varepsilon}} C_{\varepsilon 2} \rho \varepsilon
$$

and

$$
C_{\mu}=\frac{1}{A_{0}+A_{s} U^{(*)} \frac{k}{\varepsilon}}
$$

where

$$
U^{(*)}=\sqrt{S_{i j} S_{i j}-\Omega_{i j} \Omega_{i j}}, A_{s}=\sqrt{6} \cos \phi, \phi=\frac{1}{3} \cos ^{-1}(\sqrt{6} W), W=\frac{S_{i j} S_{j k} S_{k i}}{\left(\sqrt{S_{i j} S_{i j}}\right)^{3}}, A_{0}=4.0
$$

the coefficient $\mathrm{C}_{\varepsilon 1}$ is expressed as:

$$
C_{\varepsilon 1}=\max (0.43, \eta / 5+\eta)
$$

where $\eta=S k / \varepsilon$, and the rest of the coefficients are: $C_{\varepsilon 2}=1.9, \sigma_{k}=1.0, \sigma_{\varepsilon}=1.2$.

For Two-layer formulations, the transport equation of $k$ (equation (9)) is applied for inner and outer layers, while for $\varepsilon$ the transport equation (19) is only applied for the outer layer because of using algebraic equation for the outer layer, defined as:

$$
\varepsilon=\mathrm{k}^{3 / 2} / \mathrm{l}_{\varepsilon}
$$

The merging function suggested by Jongen [42] is:

$$
\lambda=0.5\left[1+\tanh \left\{\left(\operatorname{Re}_{\mathrm{y}}-\mathrm{Re}_{\mathrm{y}}{ }^{*}\right) / \mathrm{A}\right\}\right]
$$


$\operatorname{Re}_{\mathrm{y}}{ }^{*}$ specifies the limit of applicability of the two-layer formulation. A value of $\operatorname{Re}_{\mathrm{y}}{ }^{*}=60$ is used in the code. The width of the merging function is determined by the constant $\mathrm{A}$. By giving a width such that the value of $\lambda$ is within $1 \%$ of its value far from a wall for a given difference of $\Delta \mathrm{Re}_{\mathrm{y}}$, the following relation between $\mathrm{A}$ and $\Delta \operatorname{Re}_{\mathrm{y}}$ is obtained: $\mathrm{A}=$ $\left|\Delta \operatorname{Re}_{y}\right| / \tanh ^{-1}(0.98)$ and $\Delta \operatorname{Re}_{\mathrm{y}}=10$.

the turbulent Reynolds number:

$$
R e_{y}=\sqrt{k} y / v
$$

$\mathrm{y}$ is the normal distance to the wall.

The length scale is defined by the one-equation model of Wolfstein [41] is:

$$
\mathrm{l}_{\varepsilon}=\mathrm{c}_{\mathrm{l}} \mathrm{y}\left[1-\exp \left(\mathrm{Re}_{\mathrm{y}} / \mathrm{A}_{\varepsilon}\right)\right]
$$

where $A_{\varepsilon}=2 c_{l}, c_{l}=\kappa C_{\mu}^{-3 / 4}, C_{\mu}=0.09$, and $\kappa=0.42$.

The turbulent viscosity for the inner layer is defined as:

$$
\mu_{\mathrm{t}} / \mu=\operatorname{Re}_{\mathrm{y}} \mathrm{C}_{\mu}^{1 / 4} \kappa\left[1-\exp \left(-\operatorname{Re}_{\mathrm{y}} / 70\right)\right]
$$

The merging function of the eddy viscosity from both the $(k-\varepsilon)$ and two-layer models is:

$$
\mu_{\mathrm{t}}=\left.\lambda \mu_{\mathrm{t}}\right|_{\mathrm{k}-\varepsilon}+(1-\lambda) \mu\left(\mu_{\mathrm{t}} / \mu\right)_{2 \text { layer }}
$$

\section{A.3. $(k-\varepsilon) L L R \&(k-\varepsilon) L L R N Q$ models}

The transport equation for $k$ is the same as in equations (9), and for $\varepsilon$ is defined as:

$$
\frac{\partial(\rho \varepsilon)}{\partial t}+\frac{\partial}{\partial x_{j}}\left(\rho U_{j} \varepsilon\right)=\frac{1}{T}\left[C_{\varepsilon 1}\left(P_{k}+P^{\prime}\right)-C_{\varepsilon 2} f_{2} \rho \varepsilon\right]+\frac{\partial}{\partial x_{j}}\left[\left(\mu+\frac{\mu_{t}}{\sigma_{\varepsilon}}\right) \frac{\partial \varepsilon}{\partial x_{j}}\right]
$$

where $P^{\prime}$ is an additional production term, given by equation (12)

$f_{2}$ is a damping function, defined as:

$$
f_{2}=1-0.3 \exp \left(R e_{t}^{2}\right)
$$

where $\operatorname{Re}_{\mathrm{y}}$ and $\mathrm{Re}_{\mathrm{t}}$ are turbulence Reynolds number, in which $\mathrm{Re}_{\mathrm{y}}$ is given by equation (25), and $\operatorname{Re}_{\mathrm{t}}$ :

$$
R e_{t}=k^{2} / \varepsilon v
$$

the eddy-viscosity is defined in equation (6) with the damping function $f_{\mu}$ given in equation (13).

and the constants are:

$C_{d 1}=0.091, C_{d 2}=0.0042, C_{d 3}=0.00011$

The coefficients for $(k-\varepsilon) L L R$ model are:

$\mathrm{C}_{\varepsilon 1}=1.44, \mathrm{C}_{\varepsilon 2}=1.92, \mathrm{C}_{\mu}=0.09, \sigma_{\mathrm{k}}=1.0, \sigma_{\varepsilon}=1.3, \mathrm{C}_{\mathrm{t}}=1.0$

The same coefficients are used for $(k-\varepsilon) L L R N Q$ except $\mathrm{C}_{\mu}$ which is defined in equation (19).

\section{A.4. Shear Stress Transport SST $(\boldsymbol{k}-\omega)$ Model}

The following transport equations are employed to compute $k$ and $\omega$ : 


$$
\begin{aligned}
& \frac{\partial(\rho k)}{\partial t}+\frac{\partial}{\partial x_{j}}\left(\rho U_{j} k\right)=P_{k}-\rho \beta^{*} \omega k+\frac{\partial}{\partial x_{j}}\left[\left(\mu+\sigma_{k} \mu_{t}\right) \frac{\partial k}{\partial x_{j}}\right] \\
& \frac{\partial(\rho \omega)}{\partial t}+\frac{\partial}{\partial x_{j}}\left(\rho U_{j} \omega\right)=P_{\omega}-\rho \beta \omega^{2}+D_{\omega}+\frac{\partial}{\partial x_{j}}\left[\left(\mu+\sigma_{\omega} \mu_{t}\right) \frac{\partial \omega}{\partial x_{j}}\right]
\end{aligned}
$$

the production of $\omega$ is defined as:

$$
P_{\omega}=\rho \gamma\left[\left(S^{2}-\frac{2}{3}\left(\frac{\partial U_{j}}{\partial x_{j}}\right)^{2}\right)-\frac{2}{3} \omega \frac{\partial U_{j}}{\partial x_{j}}\right]
$$

the term $\mathrm{D}_{\omega}$ is a cross - derivative term, described as:

$$
D_{\omega}=2\left(1-F_{1}\right) \rho \sigma_{\omega 2} \frac{1}{\omega} \frac{\partial k}{\partial x_{j}} \frac{\partial \omega}{\partial x_{j}}
$$

$F_{1}$ is the blending function given as a function of the distance to the wall as:

$$
F_{1}=\tanh \left(\arg _{1}^{4}\right)
$$

with

$$
\arg _{1}=\min \left(\max \left(\sqrt{k} / 0.09 \omega y, 500 v / y^{2} \omega\right), 2 k / y^{2} C D_{k \omega}\right)
$$

Where

$$
C D_{k \omega}=\max \left(\frac{1}{\omega} \frac{\partial k}{\partial x_{j}} \frac{\partial \omega}{\partial x_{j}} ; 10^{-20}\right)
$$

The coefficients in the model are calculated from the blending function $F_{1}$, such that each coefficient $\phi$ is defined as:

$$
\phi=F_{1} \phi_{1}+\left(1-F_{1}\right) \phi_{2}
$$

where $\phi_{1}$ for values from $(k-\omega)$ model and $\phi_{2}$ for $(k-\varepsilon)$ model. The coefficients of Set $1\left(\phi_{1}\right)$ are:

$\beta_{1}=0.075, \sigma_{\mathrm{k} 1}=0.85, \sigma_{\omega 1}=0.5, \kappa=0.41, \gamma_{1}=\beta_{1} / \beta^{*}-\sigma_{\omega 1} \kappa^{2} / \sqrt{\beta^{*}}$

The coefficients of Set $2\left(\phi_{2}\right)$ are:

$\beta_{2}=0.0828, \sigma_{\mathrm{k} 2}=1.0, \sigma_{\omega 2}=0.856, \kappa=0.41, \gamma_{2}=\beta_{2} / \beta^{*}-\sigma_{\omega 2} \kappa^{2} / \sqrt{\beta^{*}}$

and in both Set 1 and Set 2:

$\beta^{*}=0.09, \quad \alpha^{*}=1.0$

The turbulent viscosity is defined as:

$$
\mu_{t}=\rho k \min \left[\alpha^{*} / \omega, a_{1} / S F_{2}\right]
$$

where $S$ is the mean strain rate given as:

$$
S=\sqrt{2 S_{i j} S_{i j}}
$$

and $F_{2}$ is the second blending function given as:

$$
\mathrm{F}_{2}=\tanh \left(\arg _{2}^{2}\right)
$$

with 


$$
\arg _{2}=\max \left(2 \sqrt{\mathrm{k}} / \beta^{*} \omega \mathrm{y}, 500 \mathrm{v} / \mathrm{y}^{2} \omega\right)
$$

and $\mathrm{a}_{1}=0.31$.

\section{A.5. $\mathrm{v}^{2}-f(k-\omega)$ Model}

Here again the transport equation of $k$ is the same as equation (9), and for the specific dissipation rate $\varepsilon$, the normal velocity fluctuating $\overline{\mathrm{v}^{2}}$, and the elliptic function $f$ are:

$$
\begin{gathered}
\frac{\partial(\rho \varepsilon)}{\partial t}+\frac{\partial}{\partial x_{j}}\left(\rho U_{j} \varepsilon\right)=\rho C_{\varepsilon 1}\left(1+a \sqrt{k / \overline{\mathrm{v}^{2}}}\right) \frac{P_{k}}{T}-\rho C_{\varepsilon 2} \frac{\varepsilon^{2}}{k}+\frac{\partial}{\partial x_{j}}\left[\left(\mu+\frac{\mu_{t}}{\sigma_{\varepsilon}}\right) \frac{\partial \varepsilon}{\partial x_{j}}\right] \\
\frac{\partial\left(\rho \overline{\mathrm{v}^{2}}\right)}{\partial t}+\frac{\partial}{\partial x_{j}}\left(\rho U_{j} \overline{\mathrm{v}^{2}}\right)=\rho \min \left\{k f,-\frac{1}{T}\left[\left(C_{1}-6\right) \overline{\mathrm{v}^{2}}-\frac{2 k}{3}\left(C_{1}-1\right)\right]+C_{2} P_{k}\right\}-\frac{6 \rho \overline{\mathrm{v}^{2}} \varepsilon}{k}+\frac{\partial}{\partial x_{j}}\left[\left(\mu+\frac{\mu_{t}}{\sigma_{k}}\right) \frac{\partial \overline{\mathrm{v}^{2}}}{\partial x_{j}}\right] \\
\frac{\partial}{\partial x_{j}}\left[L^{2} \frac{\partial f}{\partial x_{j}}\right]-f+\frac{1}{T}\left(C_{1}-1\right)\left(\frac{2}{3}-\frac{\overline{\mathrm{v}^{2}}}{k}\right)+C_{2} \frac{C_{\mu_{\vartheta^{2}} \overline{\mathrm{v}}^{2}} S^{2}}{\varepsilon}+\frac{5 \overline{\mathrm{v}^{2}}}{k T}+S_{f}=0
\end{gathered}
$$

The turbulent viscosity is defined as:

$$
\mu_{t}=\rho \operatorname{Tmin}\left(C_{\mu_{s t d}} k, C_{\mu_{\vartheta^{2}}} \overline{\mathrm{v}^{2}}\right)
$$

The turbulent length scale is given as:

$$
L=C_{L} \max \left[\min \left(k^{3 / 2} / \varepsilon, k^{3 / 2} / \sqrt{3} C_{\mu_{\vartheta^{2}}} \overline{\mathrm{v}^{2}} S\right), C_{\eta}\left(v^{3} / \varepsilon\right)^{1 / 4}\right]
$$

and the turbulent time scale is described as:

$$
T=\max \left(k / \varepsilon, C_{t} \sqrt{v / \varepsilon}\right)
$$

The model coefficients are:

$\mathrm{a}=0.045, \mathrm{C}_{\varepsilon 1}=1.4, \mathrm{C}_{\varepsilon 2}=1.9, \mathrm{C}_{\mu_{\text {std }}}=0.09, \mathrm{C}_{\mu_{g^{2}}}=0.22, \sigma_{\mathrm{k}}=1.0$,

$\sigma_{\varepsilon}=1.3, \mathrm{C}_{1}=1.4, \mathrm{C}_{2}=0.3, \mathrm{C}_{\eta}=70.0, \mathrm{C}_{\mathrm{t}}=6.0, \mathrm{C}_{\mathrm{L}}=0.23$.

\section{A.6. Reynolds Stress Model-Gibson and Launder RSM-GL}

The linear pressure-strain correlation of $\phi_{i j}$ in RSM-GL consists of for main terms as:

$$
\begin{aligned}
& \phi_{i j}=\phi_{i j, 1}+\phi_{i j, 2}+\phi_{i j, w 1}+\phi_{i j, w 2} \\
& \phi_{i j, 1}=-\mathrm{C}_{s 1} \rho \frac{\varepsilon}{k}\left(\overline{u_{i} u_{j}}-(2 / 3) \delta_{i j} k\right) \\
& \phi_{i j, 2}=-\mathrm{C}_{s 2}\left[\mathrm{P}_{i j}-(1 / 3) \delta_{i j} \mathrm{P}_{k k}\right] \\
& \phi_{i j, w 1}=\mathrm{C}_{1 \mathrm{w}} \varepsilon / k\left(\overline{u_{k} u_{m}} n_{k} n_{m} \delta_{i j}-3 / 2 \overline{u_{i} u_{k}} n_{j} n_{k}-3 / 2 \overline{u_{j} u_{k}} n_{i} n_{k}\right) f_{w} \\
& \phi_{i j, w 2}=\mathrm{C}_{2 \mathrm{w}}\left(\phi_{k m, 2} n_{k} n_{m} \delta_{i j}-3 / 2 \phi_{i k, 2} n_{j} n_{k}-3 / 2 \phi_{j k, 2} n_{i} n_{k}\right) f_{w} \\
& f_{w}=\min \left(k^{3 / 2} / \mathrm{C}_{l} \varepsilon \mathrm{d}, 1.4\right)
\end{aligned}
$$

where $\mathrm{d}$ is the normal distance to the wall, $n_{k}$ is the $\mathrm{x}_{k}$ component of the unit normal to the wall, and the model coefficients are: 
$\mathrm{C}_{\mathrm{s} 1}=1.8, \mathrm{C}_{\mathrm{s} 2}=0.6, \mathrm{C}_{1 \mathrm{w}}=0.5, \mathrm{C}_{2 \mathrm{w}}=0.3, \mathrm{C}_{l}=2.5, \sigma_{k}=0.82, \mathrm{C}_{\mu}=0.09$.

\section{A.7. Reynolds Stress Model-Speziale, Sarkar and Gatski RSM-SSG}

The quadratic pressure-strain correlation of $\phi_{i j}$ in RSM-SSG is:

$$
\begin{gathered}
\phi_{i j}=-\left(\mathrm{C}_{s 1} \rho \varepsilon+\mathrm{C}_{r 4} \mathrm{P}_{k k}\right) b_{i j}+\mathrm{C}_{s 2} \rho \varepsilon\left(b_{i k} b_{k j}-1 / 3 b_{m n} b_{m n} \delta_{i j}\right)+\left(\mathrm{C}_{r 3}-\mathrm{C}_{r 3}^{*} \sqrt{b_{i j} b_{i j}}\right) \rho k \mathrm{~S}_{i j}+ \\
\mathrm{C}_{r 1} \rho k\left(b_{i k} \mathrm{~S}_{j k}+b_{j k} \mathrm{~S}_{i k}-2 / 3 b_{m n} \mathrm{~S}_{m n} \delta_{i j}\right)+\mathrm{C}_{r 2} \rho k\left(b_{i k} \Omega_{j k}+b_{j k} \Omega_{i k}\right)
\end{gathered}
$$

$b_{i j}$ is the Reynolds stress anisotropy tensor which is defined as:

$$
b_{i j}=\overline{u_{i} u_{j}} / k-(2 / 3) \delta_{i j}
$$

and the constants are:

$\mathrm{C}_{s 1}=1.7, \mathrm{C}_{r 4}=0.45, \mathrm{C}_{s 2}=1.05, \mathrm{C}_{r 3}=0.8, \mathrm{C}_{r 3}^{*}=0.65, \mathrm{C}_{r 1}=0.625, \mathrm{C}_{r 2}=0.2$.

\section{References}

[1] Global Wind Energy Council (2015). Global wind report 2014: Annual Market Update. Brussels, Belgium.

[2] Xu, G., \& Sankar, L.N. (2002). Application of a viscous flow methodology to the NREL phase VI rotor. ASME 2002 Wind Energy Symposium, 83-93.

[3] CHAVIAROPOULOS, P., \& HANSEN, M. (2000). Investigating three-dimensional and rotational effects on wind turbine blades by means of a quasi-3D Navier-Stokes solver. J. of fluids engineering, $122,330-336$

[4] Johansen, J., \& Sørensen, N.N. (2004). Aerofoil characteristics from 3D CFD rotor computations. Wind Energy, 7, $283-294$.

[5] Langtry, R.B., Gola, J., \& Menter, F.R. (2006). Predicting 2D airfoil and 3D wind turbine rotor performance using a transition model for general CFD codes. AIAA paper, 395.

[6] Duque, E.P., Van Dam, C.P., \& Hughes, S.C. (1999). Navier-Stokes simulations of the NREL combined experiment phase II rotor. EWEC-CONFERENCE, 79-84.

[7] Simms, D.A., Fingersh, L.J., Jager, D.W., Cotrell, J.R., Schreck, S., \& Larwood, S.M. (2001). Unsteady aerodynamics experiment phase VI: wind tunnel test configurations and available data campaigns. (pp. 500-29955). NREL.

[8] Fingersh, L. J., Simms, D., Hand, M., Jager, D., Cotrell, J., Robinson, M. \& Larwood, S. (2001). Wind tunnel testing of NREL's unsteady aerodynamics experiment. AIAA paper, 35, 2001.

[9] Duque, E.P., Burklund, M.D., \& Johnson, W. (2003). Navier-Stokes and comprehensive analysis performance predictions of the NREL phase VI experiment. J. of Solar Energy Engineering, 125, 457-467.

[10] Baldwin, B.S. and Barth, T.J. (1990) A One-Equation Turbulence Transport Model for High Reynolds Number Wall-Bounded Flows. NASA TM 102847.

[11] Duque, E. P., Johnson, W., VanDam, C. P., Cortes, R., \& Yee, K. (2000). Numerical predictions of wind turbine power and aerodynamic loads for the NREL phase II combined experiment rotor (No. AIAA-2000-0038). NATIONAL AERONAUTICS AND SPACE ADMINISTRATION MOFFETT FIELD CA AMES RESEARCH CENTER.

[12] Baldwin, B. S., and Lomax, H. (1978). Thin-Layer Approximation and Algebraic Model for Separated Turbulent Flow. AIAA Paper $78-0257$.

[13] Sørensen, N.N., Michelsen, J.A., \& Schreck, S. (2002). Navier-Stokes predictions of the NREL phase VI rotor in the NASA Ames $80 \mathrm{ft} \times 120 \mathrm{ft}$ wind tunnel. Wind Energy, 5, 151-169.

[14] Li, Y., Paik, K. J., Xing, T., \& Carrica, P. M. (2012). Dynamic overset CFD simulations of wind turbine aerodynamics. Renewable Energy, 37(1), 285-298.

[15] Sezer-Uzol, N., \& Long, L.N. (2006). 3-D time-accurate CFD simulations of wind turbine rotor flow fields. AIAA paper, 394.

[16] Van Rooij, R.P.J.O.M., \& Arens, E.A. (2007). Analysis of the experimental and computational flow characteristics with respect to the augmented lift phenomenon caused by blade rotation. J. of Physics: Conference Series (Vol. 75, p. 012021)

[17] Yu, G., Shen, X., Zhu, X., \& Du, Z. (2011). An insight into the separate flow and stall delay for HAWT. Renewable Energy, 36(1), 69-76.

[18] Moshfeghi, M., Song, Y. J., \& Xie, Y. H. (2012). Effects of near-wall grid spacing on SST-k- $\omega$ model using NREL Phase VI horizontal axis wind turbine. Journal of Wind Engineering and Industrial Aerodynamics, 107, 94-105

[19] Lanzafame, R., Mauro, S., \& Messina, M. (2013). Wind turbine CFD modeling using a correlation-based transitional model. Renewable Energy, 52, $31-39$.

[20] Lynch, C. E., \& Smith, M. J. (2013). Unstructured overset incompressible computational fluid dynamics for unsteady wind turbine simulations. Wind Energy,16(7), 10331048

[21] Tachos, N. S., Filios, A. E., \& Margaris, D. P. (2010). A comparative numerical study of four turbulence models for the prediction of horizontal axis wind turbine flow. Proceedings of the Institution of Mechanical Engineers, Part C: Journal of Mechanical Engineering Science, 224(9), 1973-1979.

[22] Patankar, S.V., \& Spalding, D.B. (1972). A calculation procedure for heat, mass and momentum transfer in 3D parabolic flows. Int. J. Heat Mass Transfer, 15, 1787-1806.

[23] Klein, T. S., Craft, T. J., \& Iacovides, H. (2015). Assessment of the performance of different classes of turbulence models in a wide range of non-equilibrium flows. International Journal of Heat and Fluid Flow, 51, 229-256.

[24] Jones, W.P., \& Launder, B. (1972). The prediction of laminarization with a two-equation model of turbulence. International journal of heat and mass transfer, 15, 301-314

[25] Lien, F.S., Chen, W.L., \& Leschziner, M.A. (1996). Low-Reynolds-number eddy-viscosity modelling based on non-linear stress-strain/vorticity relations. Engineering turbulence modelling and experiments, 3, 91-100.

[26] Shih, T. H., Liou, W. W., Shabbir, A., Yang, Z., \& Zhu, J. (1995). A new k- $\varepsilon$ eddy viscosity model for high Reynolds number turbulent flows. Computers \& Fluids, 24, 227238

[27] Menter, F.R. (1994). Two-equation eddy-viscosity turbulence models for engineering applications. AIAA journal, 32, 1598-1605.

[28] Davidson, L., Nielsen, P.V., and Sveningsson, A. (2003). Modifications of the ( $\left.\mathrm{v}^{2}-f\right)$ Model for Computing the Flow in a 3D Wall Jet. Turbulence, Heat and Mass Transfer, 4, 577-584.

[29] Durbin, P.A. (1996). On the k-e stagnation point anomaly. Int. J. Heat and Fluid Flow 17, 89-90.

[30] Gibson, M. M., \& Launder, B. E. (1978). Ground effects on pressure fluctuations in the atmospheric boundary layer. Journal of Fluid Mechanics, 86, $491-511$.

[31] Speziale, C. G., Sarkar, S., \& Gatski, T. B. (1991). Modelling the pressure-strain correlation of turbulence: an invariant dynamical systems approach. J. Fluid Mechanics, 227, $245-272$.

[32] Boussinesq, J. (1877). Theorie de l'ecoulement tourbillant. Mem. Pres. par div. savant a lacad. sci. Paris, 23:46.

[33] Versteeg, H.K., and Malalasekera, W. (2007). An Introduction to Computational Fluid Dynamics: The Finite Volume Method. Longman, Harlow.

[34] Launder, B.E., \& Sharma, B.I. (1974). Application of the energy-dissipation model of turbulence to the calculation of flow near a spinning disc. Letters in heat and mass transfer, 1, 131-137.

[35] Goldberg, U., \& Apsley, D. (1997). A wall-distance-free low Re k- $€$ turbulence model. Computer methods in applied mechanics and engineering, 145, 227-238.

[36] Speziale, C.G. (1987). On nonlinear k-1 and k-e models of turbulence. J. Fluid Mech. 178, 459-475.

[37] Craft, T. J., Launder, B. E., \& Suga, K. (1996). Development and application of a cubic eddy-viscosity model of turbulence. Int. J. Heat and Fluid Flow, 17, 108-115.

[38] Azzola, J., Humphrey, J. A. C., Iacovides, H., \& Launder, B. E. (1986). Developing turbulent flow in a U-bend of circular cross-section: measurement and computation. Journal of fluids engineering, $108(2), 214-221$

[39] Iacovides, H., Launder, B. E., \& Loizou, P. A. (1987). Numerical computation of turbulent flow through a square-sectioned 90 bend. International journal of heat and fluid flow, 8(4), 320-325.

[40] Iacovides, H., \& Launder, B. E. (1987). Turbulent momentum and heat transport in square-sectioned ducts rotating in orthogonal mode. Numerical Heat Transfer, Part A:

[41] Wolfstein, M. (1969). The velocity and temperature distribution in one-dimensional flow with turbulence augmentation and pressure gradient. Int. J. Heat Mass Transfer, 12, 
301-318.

[42] Jongen, T. (1998). Simulation and Modelling of Turbulent Incompressible Flows. Ph.D. Thesis, Lausanne EPFL.

[43] Wilcox, D. C. (1988). Reassessment of the scale-determining equation for advanced turbulence models. AIAA journal, 26, $1299-1310$.

[44] Johnson, D. A., \& King, L. S. (1985). A mathematically simple turbulence closure model for attached and separated turbulent boundary layers. AIAA journal, 23, 1684-1692.

[45] Iacovides, H., Launder, B. E., \& Li, H. Y. (1996). Application of a reflection-free DSM to turbulent flow and heat transfer in a square-sectioned U-bend. Experimental Thermal and Fluid Science, 13(4), 419-429.

[46] Bo, T., Iacovides, H., \& Launder, B. E. (1995). Developing buoyancy-modified turbulent flow in ducts rotating in orthogonal mode. Journal of turbomachinery, 117(3), 474-

[47] Hanjalic, K., \& Launder, B. E. (1972). A Reynolds stress model of turbulence and its application to thin shear flows. J. fluid Mechanics, 52, 609-638.

[48] Launder, B. E., Reece, G. J., \& Rodi, W. (1975). Progress in the development of a Reynolds-stress turbulence closure. Journal of fluid mechanics, 68, 537-566.

[49] Daly, B. J., \& Harlow, F. H. (1970). Transport equations in turbulence. Physics of Fluids (1958-1988), 13, 2634-2649.

[50] Lien, F. S., \& Leschziner, M. A. (1994). Assessment of turbulence-transport models including non-linear RNG eddy- viscosity formulation and second-moment closure for flow over a backward-facing step. Computers \& Fluids, 23(8), 983-1004.

[51] Launder, B.E., Reynolds, W.C., Rodi, W. (1984). Turbulence models and their applications. In: Modeles de Turbulence et Leurs Applications. Collection de la Direction des Etudes et Recherches d'Electricite de France, vol. 2. Editions Eyrolles, Paris.

[52] E. L. Blades and D. L. Marcum. A sliding interface method for unsteady unstructured flow simulations. International Journal for Numerical Methods in Fluids, 53:507-529, 2007.

[53] Giguere, P., \& Selig, M.S. (1999). Design of a tapered and twisted blade for the NREL combined experiment rotor. NREL/SR, 500, 26173.

[54] Choi, Y. D., Iacovides, H., \& Launder, B. E. (1989). Numerical computation of turbulent flow in a square-sectioned 180 deg bend. Journal of Fluids Engineering, 111(1), 59-

[55] Craft, T. J., Iacovides, H., \& Yoon, J. H. (2000). Progress in the use of non-linear two-equation models in the computation of convective heat-transfer in impinging and separated flows. Flow, Turbulence and Combustion, 63(1-4), 59-80.

Table 1: List of Turbulent models investigated in the present study.

\begin{tabular}{|c|c|c|c|}
\hline & $\begin{array}{l}\text { Model Naming } \\
\text { Convention }\end{array}$ & Category & Description \\
\hline \multirow{10}{*}{ 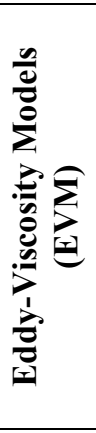 } & $\mathrm{S}(k-\varepsilon) \mathrm{HR}$ & \multirow{3}{*}{1} & Standard $(k-\varepsilon)$ High-Reynolds-number [24] \\
\hline & $(k-\varepsilon)$ LHRNQ & & $(k-\varepsilon)$ Lien High-Reynolds-number Non-linear Quadratic $[24,25]$ \\
\hline & $(k-\varepsilon)$ LHRNC & & $(k-\varepsilon)$ Lien High-Reynolds-number Non-linear Cubic $[24,25]$ \\
\hline & $\mathrm{R}(k-\varepsilon) \mathrm{HR}$ & \multirow{2}{*}{2} & Realizable $(k-\varepsilon)$ High-Reynolds-number [26] \\
\hline & $\mathrm{R}(k-\varepsilon) \mathrm{TL}$ & & Realizable $(k-\varepsilon)$ Two-Layer [26] \\
\hline & $(k-\varepsilon)$ LLR & \multirow{3}{*}{3} & $(\mathrm{k}-\varepsilon)$ Lien Low-Reynolds-Number $[25]$ \\
\hline & $(k-\varepsilon)$ LLRNQ & & $(k-\varepsilon)$ Lien Low-Reynolds-Number Non-linear Quadratic [25] \\
\hline & $(k-\varepsilon)$ TLLNQ & & $(\mathrm{k}-\varepsilon)$ Two-Layer Lien Non-linear Quadratic [25] \\
\hline & $\operatorname{SST}(k-\omega)$ & 4 & Shear Stress Transport SST $(k-\omega)[27]$ \\
\hline & $\mathrm{v}^{2}-f(k-\varepsilon)$ & 5 & $\mathrm{v}^{2}-f(k-\varepsilon)$ Low-Reynolds-number $[28,29]$ \\
\hline \multirow{2}{*}{ 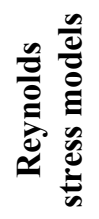 } & RSM-GL & 6 & Reynolds Stress Model-Gibson and Launder [30] \\
\hline & RSM-SSG & 7 & Reynolds Stress Model-Speziale, Sarkar and Gatski [31] \\
\hline
\end{tabular}

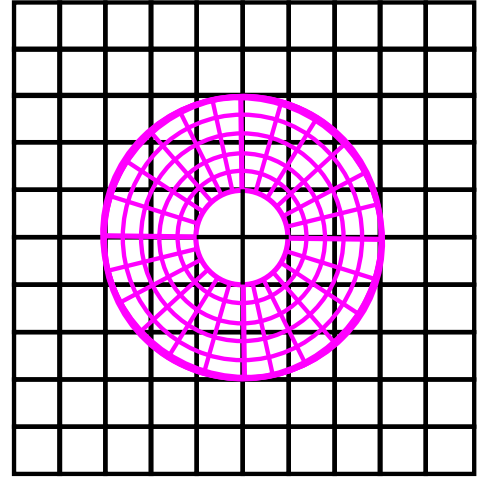

(a) Chimera

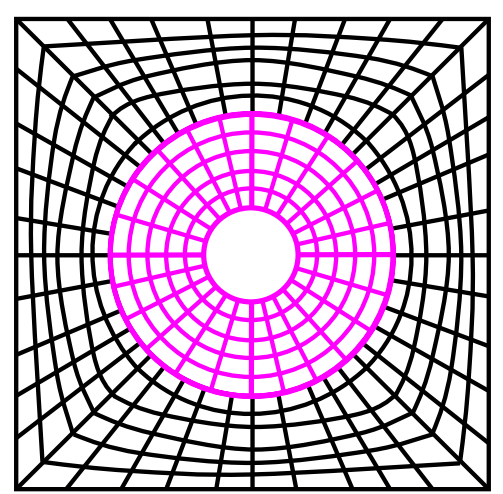

(b) Sliding mesh

Figure 1: Examples of Chimera and sliding mesh approaches

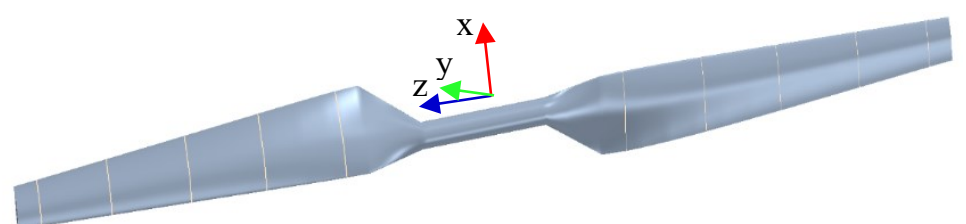

Figure 2: NREL Phase VI rotor blade geometry model 


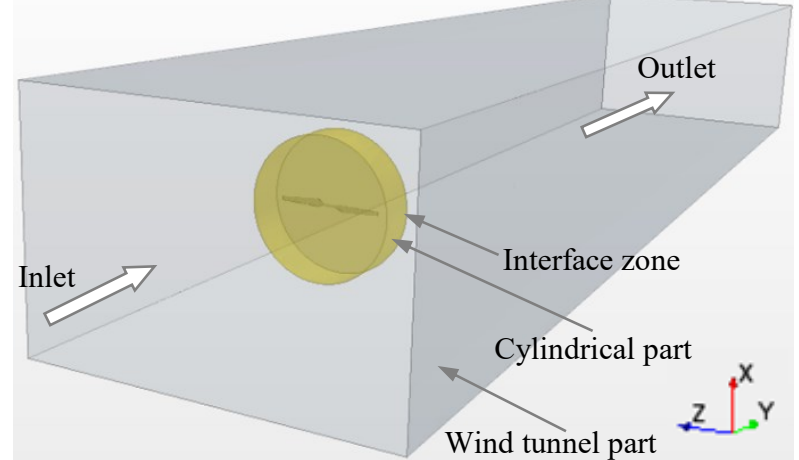

Figure 3: computational domain

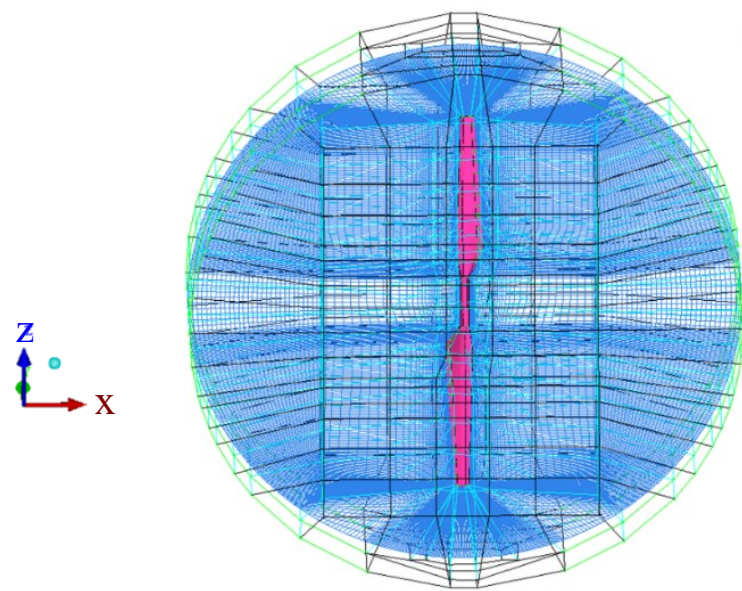

Figure 5: Blocking strategy for the cylindrical part grids and grid on slice through plane of rotation.

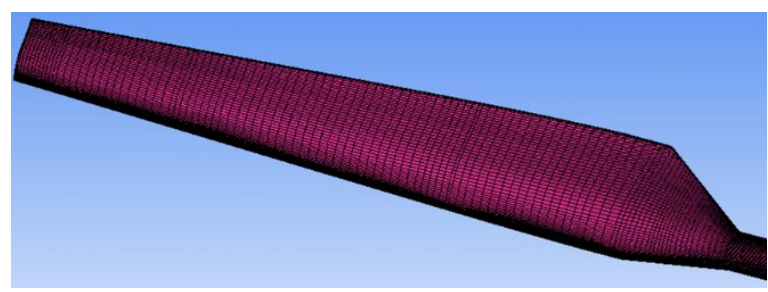

Figure 4: Grid distribution on the blade surface.

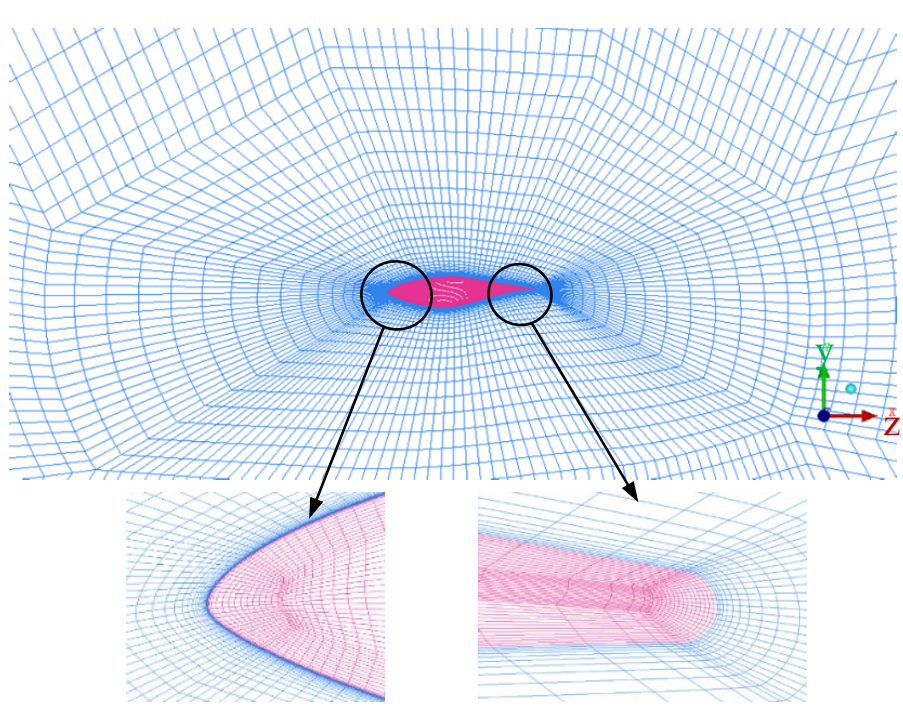

Figure 6: O-grid around the blade and on the blade tip and close up of leading- and trailing edges.
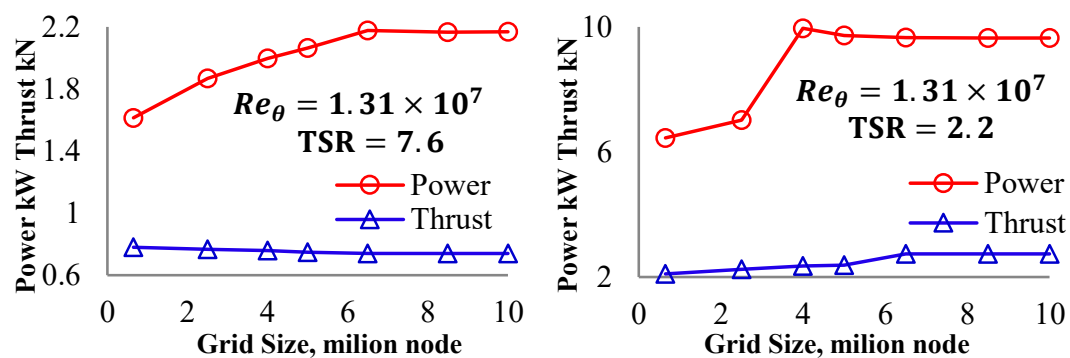

Figure 7(a): Power and thrust for tip-speed ratios 7.6 and 2.2 vs. several mesh sizes using the linear low-Re $k-\varepsilon$, LLR model.
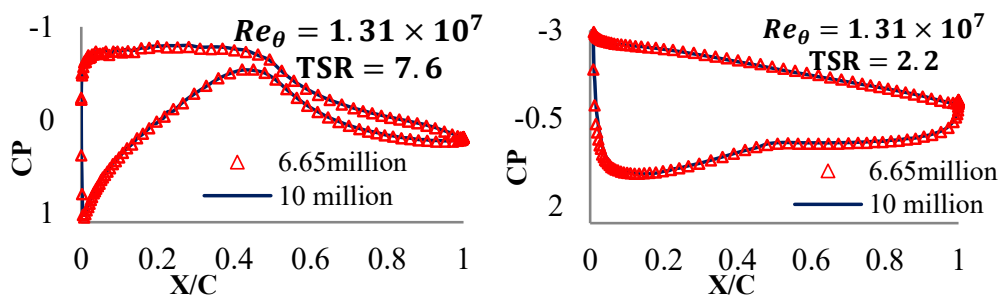

Figure 7(b): Local pressure coefficient at $r / R=0.3$, and TSR of 7.6 and 2.2, several mesh sizes using the linear low-Re $k-\varepsilon$, LLR model. 
(i)

\begin{tabular}{llllcrr}
$\mathrm{g}$ & \multicolumn{5}{c}{$\mathrm{y}^{*}$ at near-wall nodes } \\
$\mathrm{Y} Z$ & 0.00 & 0.20 & 0.40 & 0.60 & 0.80 & 1.0
\end{tabular}

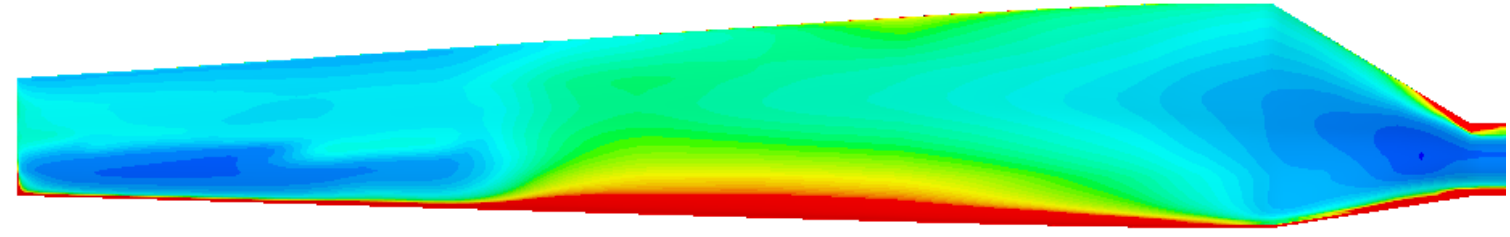

(ii)

\begin{tabular}{lllllll} 
& \multicolumn{5}{c}{$\mathrm{y}^{*}$ at near-wall nodes } \\
$\mathrm{Z}$ & 0.00 & 0.20 & 0.40 & 0.60 & 0.80 & 1.0 \\
\hline
\end{tabular}

Figure 8(a): The $\mathrm{y}^{*}$ contour of the nodes in the vicinity of (i) the suction side of the blade surface and (ii) the pressure side of the blade surface, for the grids selected for low-Reynolds-number models at the lowest tip-speed ratio or wind-speed $25 \mathrm{~m} / \mathrm{s}$.

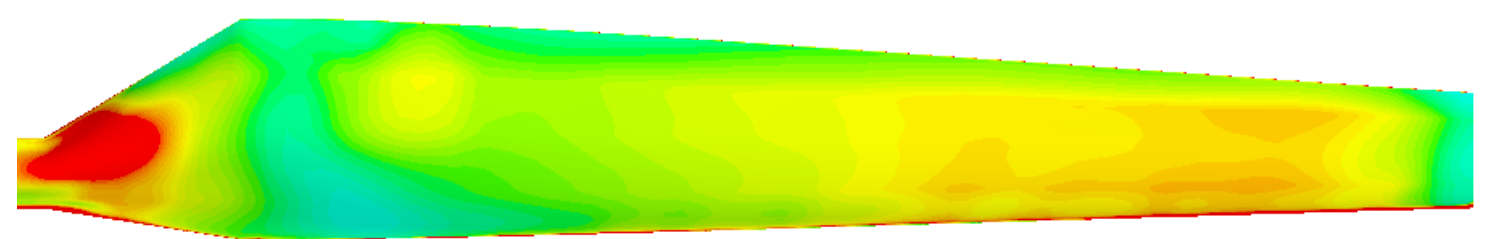

(i)
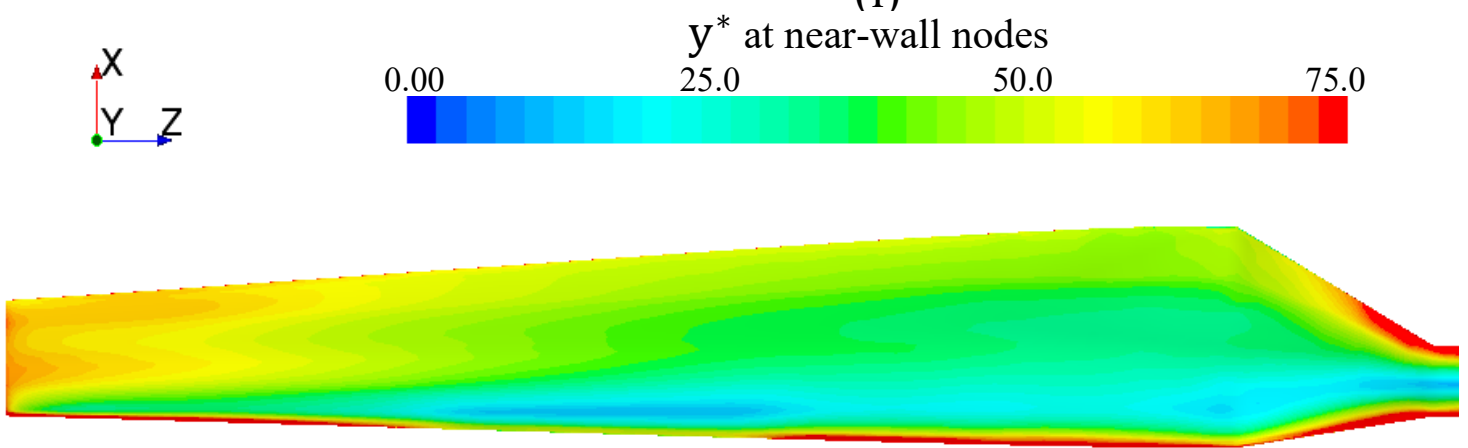

(ii)

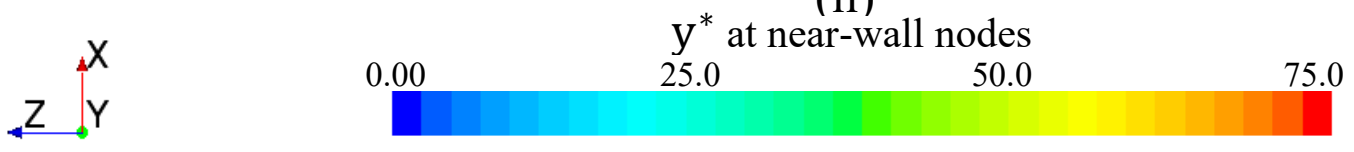

Figure $8(\mathrm{~b})$ : The $\mathrm{y}^{*}$ contour of the nodes in the vicinity of (i) the suction side of the blade surface and (ii) the pressure side of the blade surface, for the grids selected for high-Reynolds-number models at the lowest tip-speed ratio or wind-speed $25 \mathrm{~m} / \mathrm{s}$. 

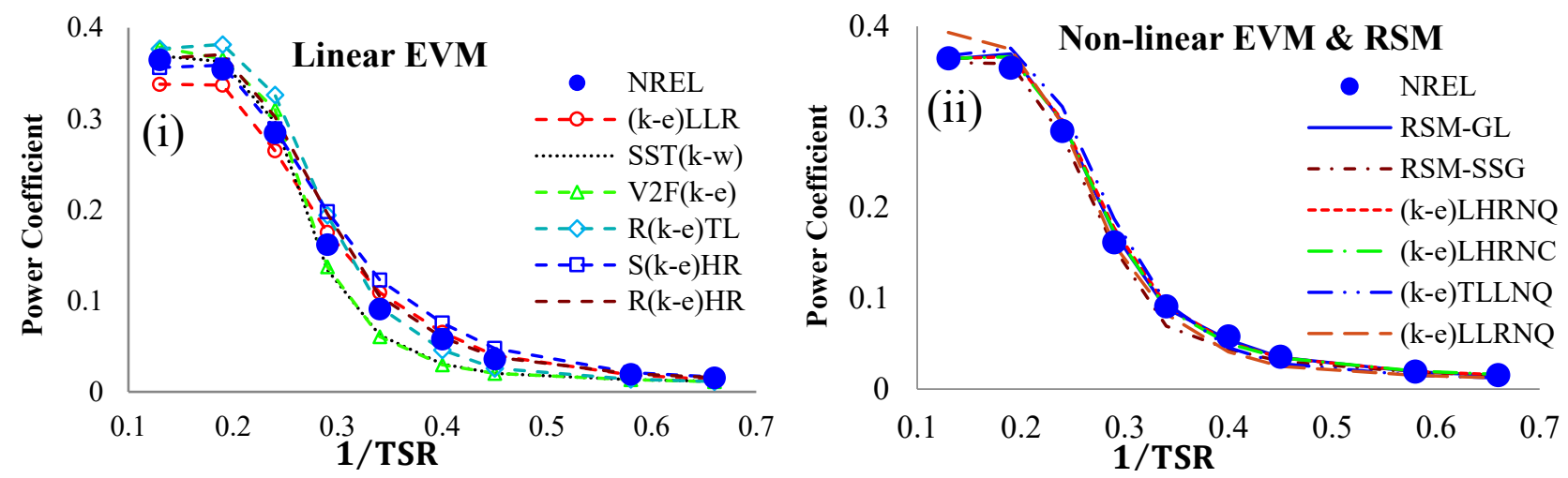

Figure (9a): Power coefficient at different inverse tip-speed ratios using (i) linear EVM and (ii) non-linear EVM and RSM.
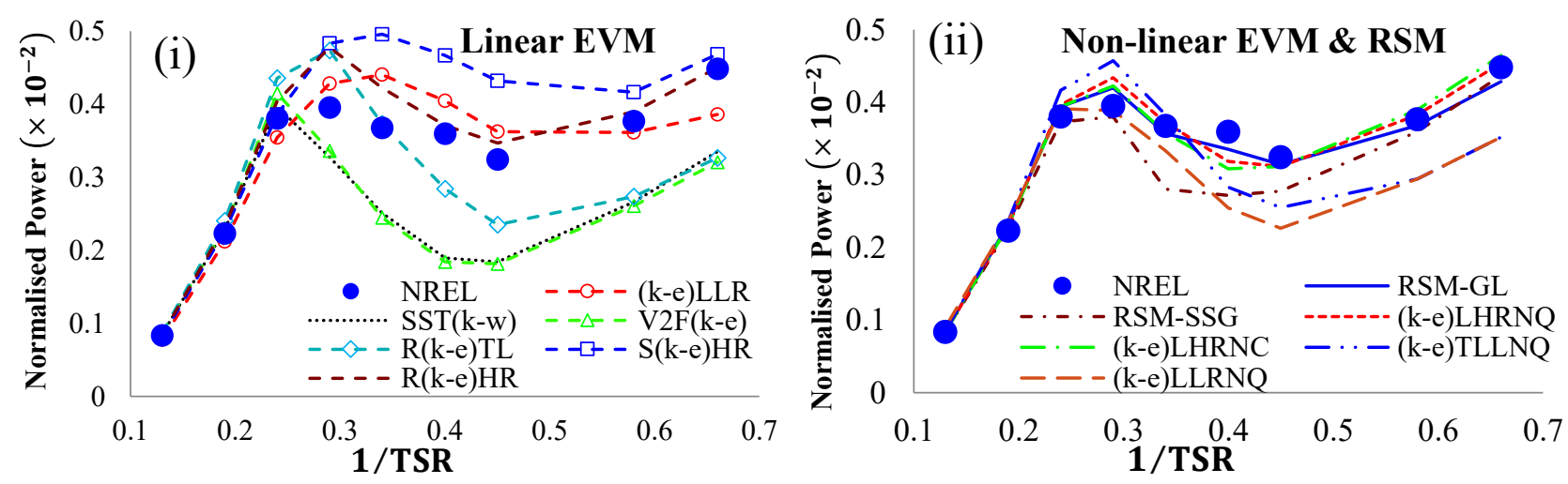

Figure 9b: Aerodynamic normalised power vs inverse tip-speed ratio at rotational Reynolds-number $\boldsymbol{R e}_{\boldsymbol{\theta}}=\mathbf{1 . 3 1} \times \mathbf{1 0}^{\mathbf{7}}$ using (i) linear EVM, and (ii) non-linear EVM and RSM.

Table 2: The range of wind speeds and corresponding tip-speed ratios included in the present study

\begin{tabular}{|c|c|c|c|c|c|c|c|c|}
\hline Wind Speeds (m/s) & 5 & 9 & 11 & 13 & 15 & 17 & 22 & 25 \\
\hline tip-speed ratio (TSR) & 7.6 & 4.2 & 3.5 & 2.9 & 2.5 & 2.2 & 1.7 & 1.5 \\
\hline
\end{tabular}



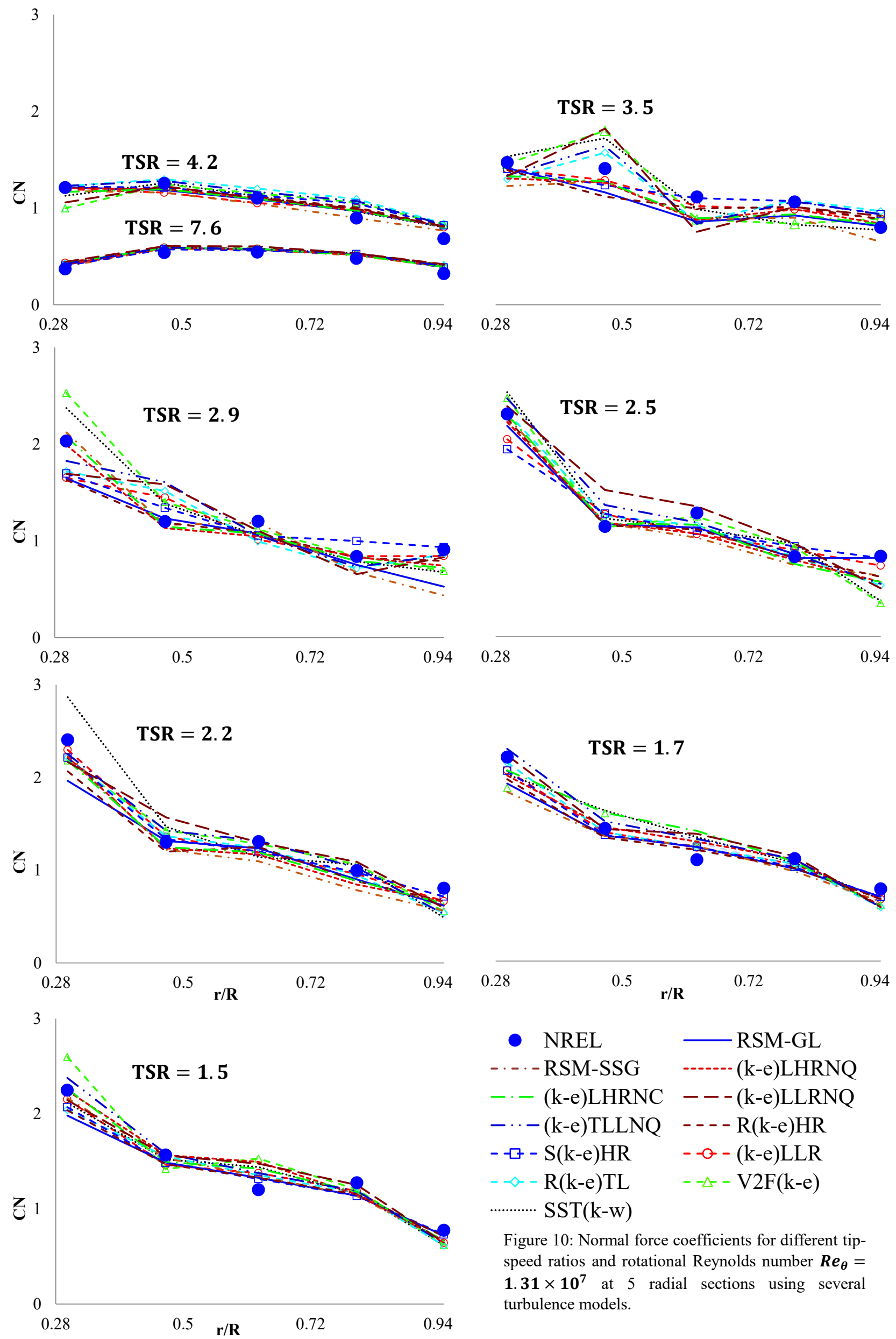

Figure 10: Normal force coefficients for different tipspeed ratios and rotational Reynolds number $\boldsymbol{R e}_{\boldsymbol{\theta}}=$ $\mathbf{1 . 3 1} \times \mathbf{1 0}^{\mathbf{7}}$ at 5 radial sections using several turbulence models. 

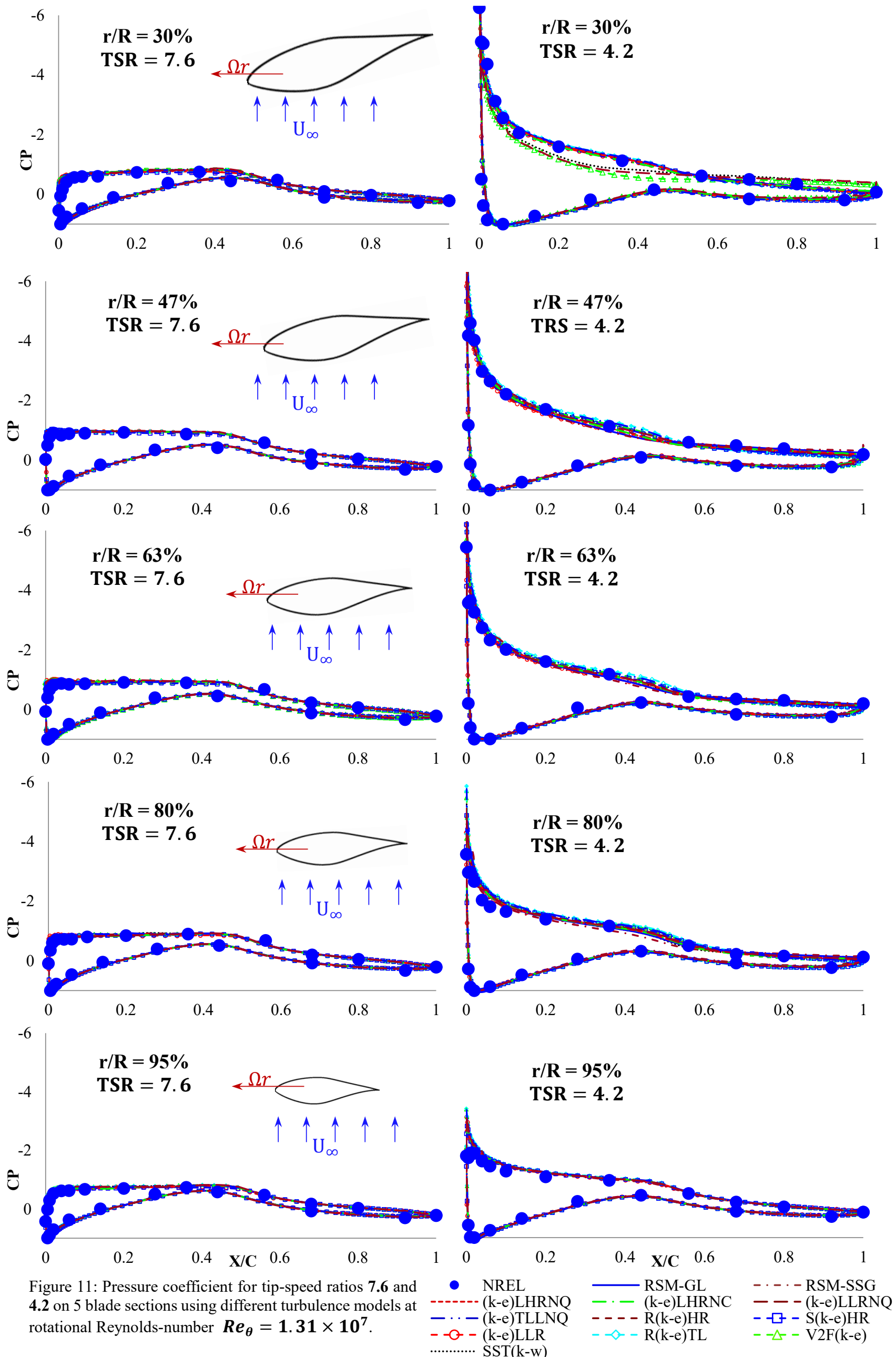

Figure 11: Pressure coefficient for tip-speed ratios $\mathbf{7 . 6}$ and 4.2 on 5 blade sections using different turbulence models at rotational Reynolds-number $\boldsymbol{R e}_{\boldsymbol{\theta}}=\mathbf{1 . 3 1} \times \mathbf{1 0}^{\mathbf{7}}$.

$-\cdots-(\mathrm{k}-\mathrm{e}) \mathrm{TLLN} Q$ 

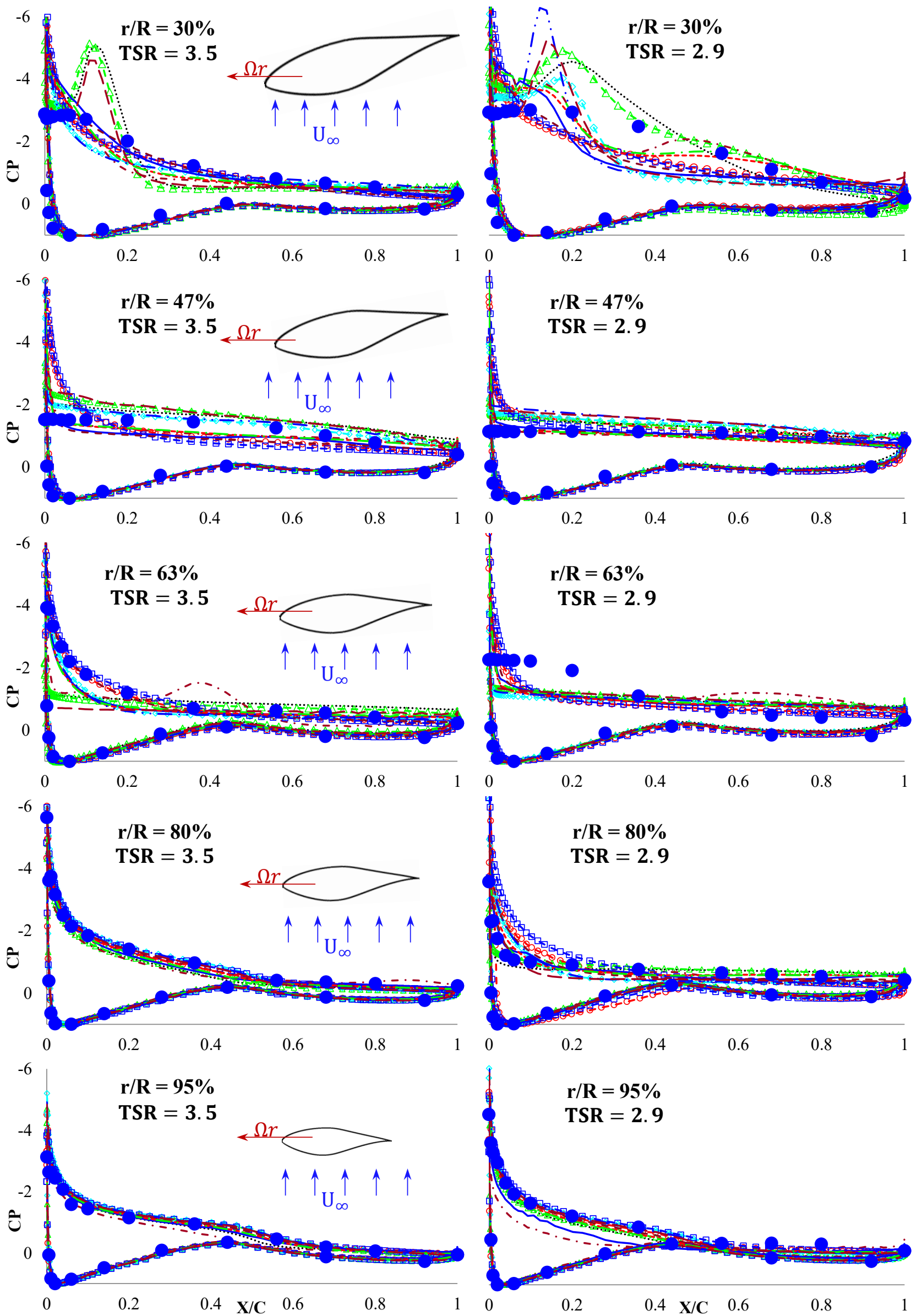

Figure 12: Pressure coefficient for tip-speed ratios $\mathbf{3 . 5}$ and 2.9 on 5 blade sections using different turbulence models at rotational Reynolds-number $\boldsymbol{R e}_{\boldsymbol{\theta}}=\mathbf{1 . 3 1} \times \mathbf{1 0}^{\mathbf{7}}$.

$$
\begin{aligned}
& \text { - NREL — RSM-GL - - - RSM-SSG } \\
& \text {--.-- (k-e)LHRNQ - - (k-e)LHRNC - - (k-e)LLRNQ } \\
& -\cdots-(\mathrm{k}-\mathrm{e}) \mathrm{TLLNQ} \quad---\mathrm{R}(\mathrm{k}-\mathrm{e}) \mathrm{HR} \quad--\mathrm{-}-\mathrm{S}(\mathrm{k}-\mathrm{e}) \mathrm{HR} \\
& --\mathrm{-}-(\mathrm{k}-\mathrm{e}) \mathrm{LLR} \quad-\diamond-\mathrm{R}(\mathrm{k}-\mathrm{e}) \mathrm{TL} \quad--\triangle-\mathrm{V} 2 \mathrm{~F}(\mathrm{k}-\mathrm{e})
\end{aligned}
$$



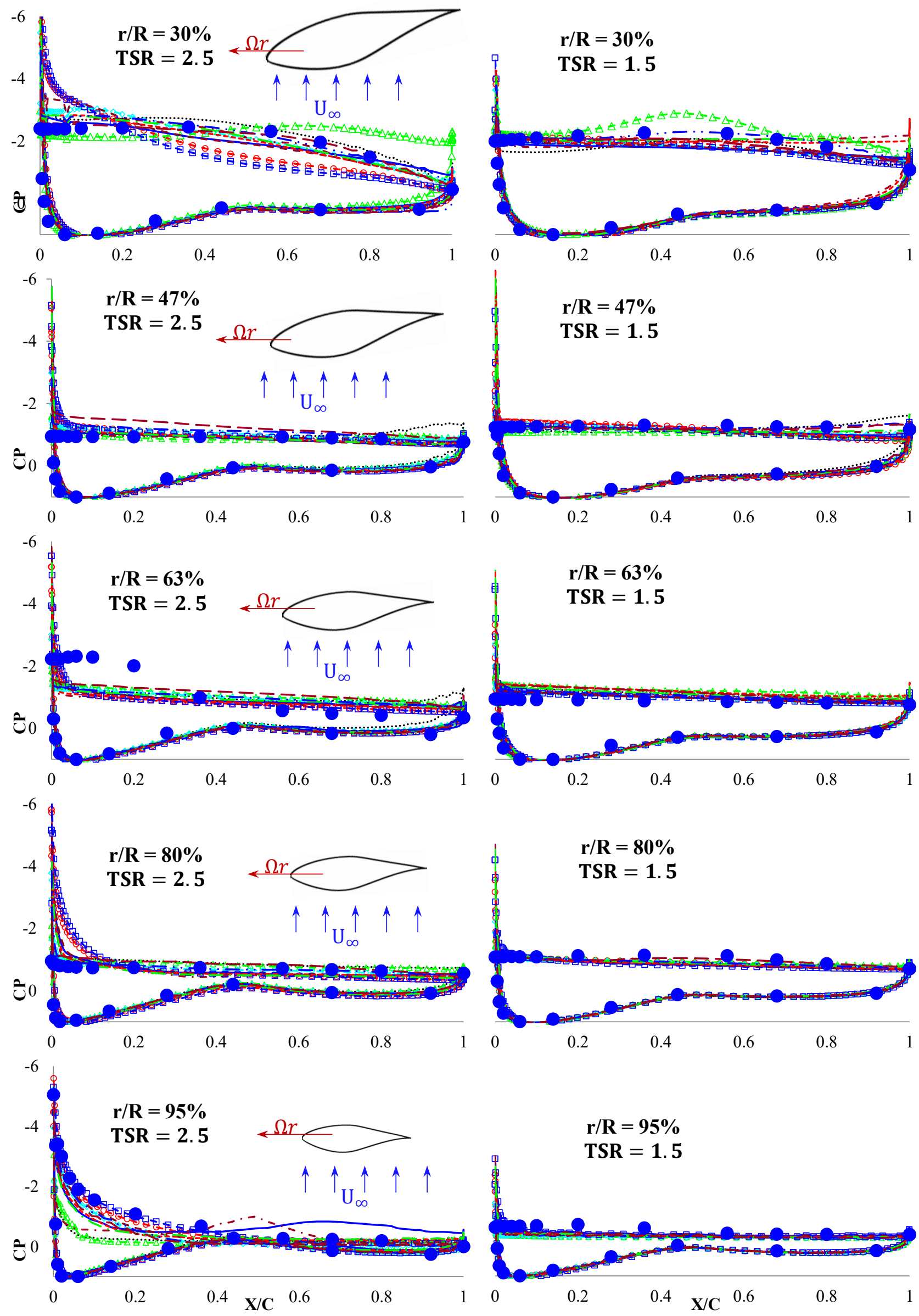

Figure 13: Pressure coefficient for tip-speed ratios 2.5 and $\mathbf{1 . 5}$ on 5 blade sections using different turbulence models at rotational Reynolds-number $\boldsymbol{R e}_{\boldsymbol{\theta}}=\mathbf{1 . 3 1} \times \mathbf{1 0}^{\mathbf{7}}$.

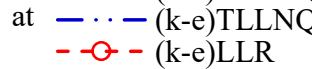

$$
\begin{aligned}
& r / R=95 \% \\
& T S R=1.5
\end{aligned}
$$

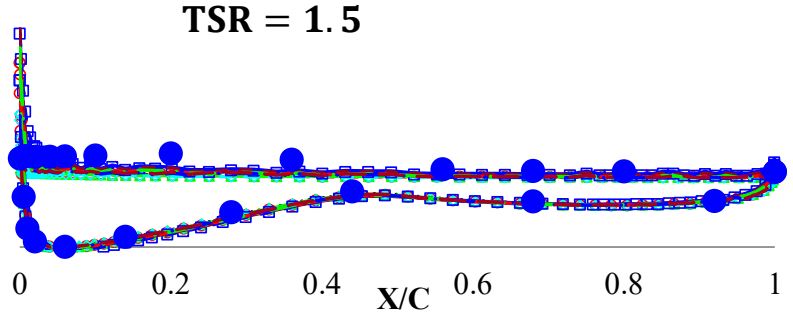

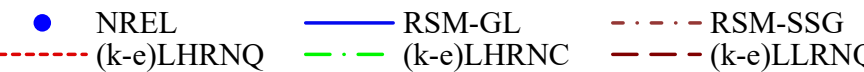
-..-(k-e)TLLNQ - - - - R(k-e)HR - - - S $(\mathrm{k}-\mathrm{e}) \mathrm{HR}$ ….... SST $(k-w)$ 


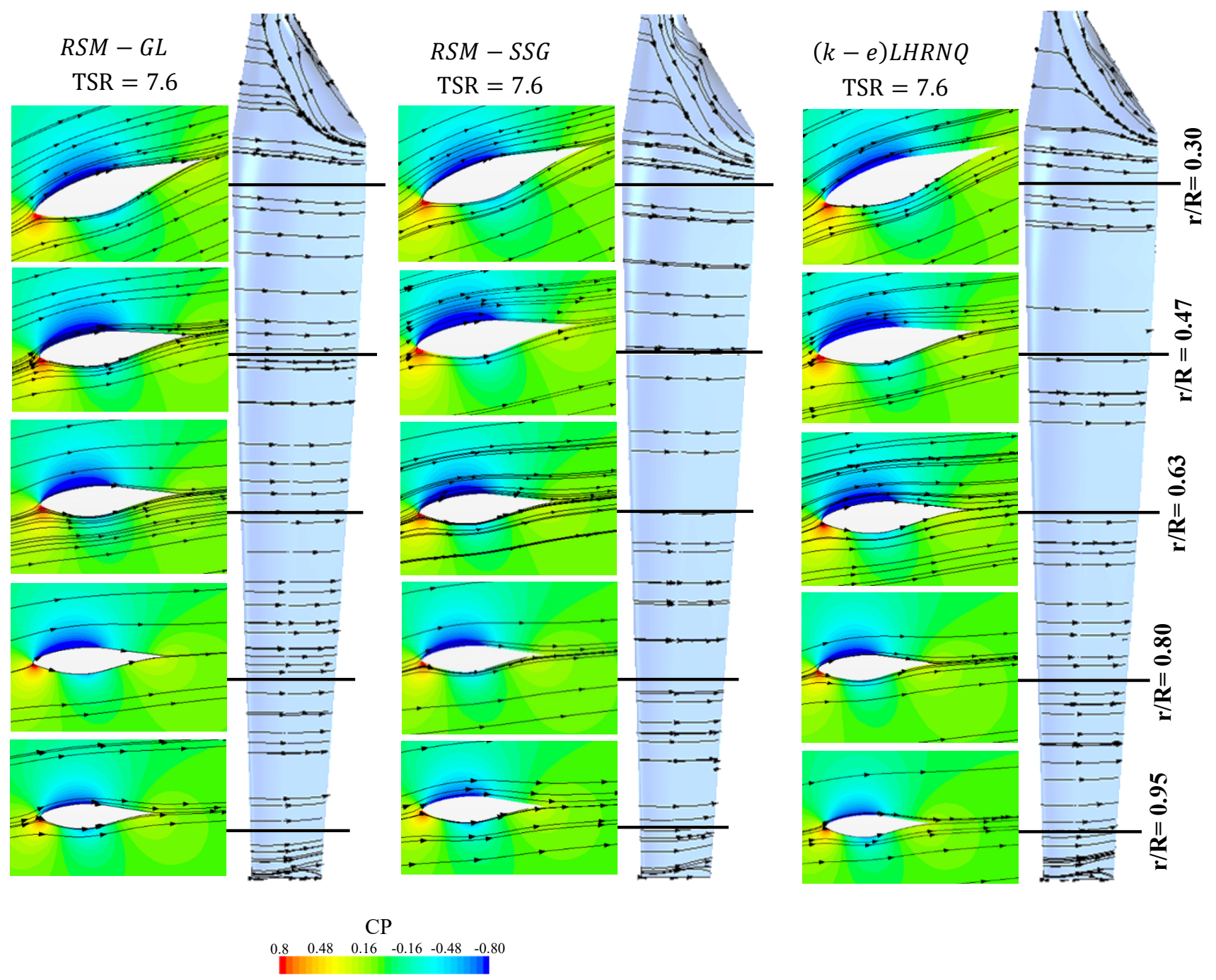

Figure 14: Instantaneous flow path lines on the upper (downwind) side of the blade and five spanwise sections for tip-speed ratio 7.6 and three turbulence models at rotational Reynolds-number $1.31 \times 10^{7}$. 

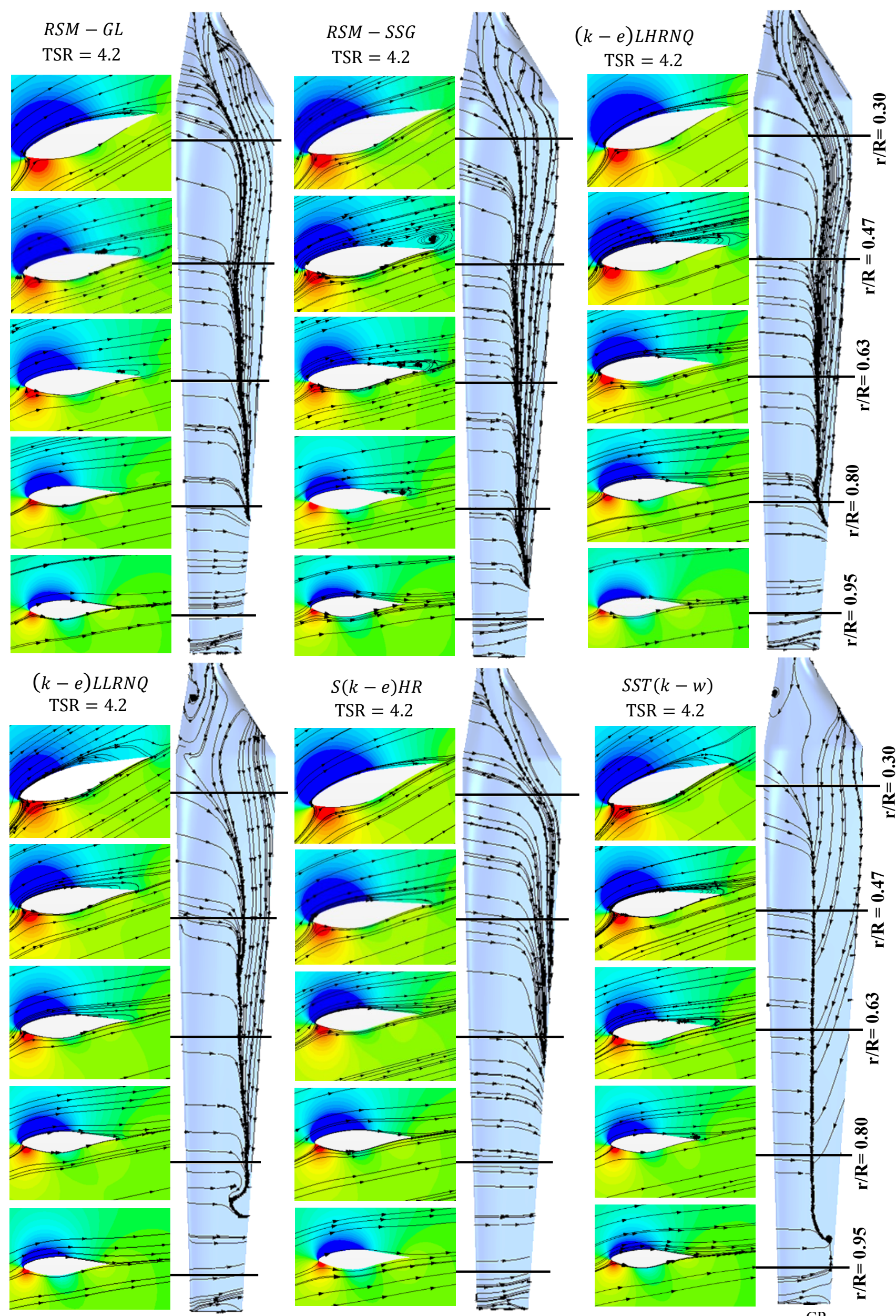

Figures 15: Instantaneous flow path lines on the upper (downwind) side of the blade and five spanwise sections for tip-speed ratio 4.2 and six turbulence models at rotational Reynolds-number $1.31 \times 10^{7}$. 


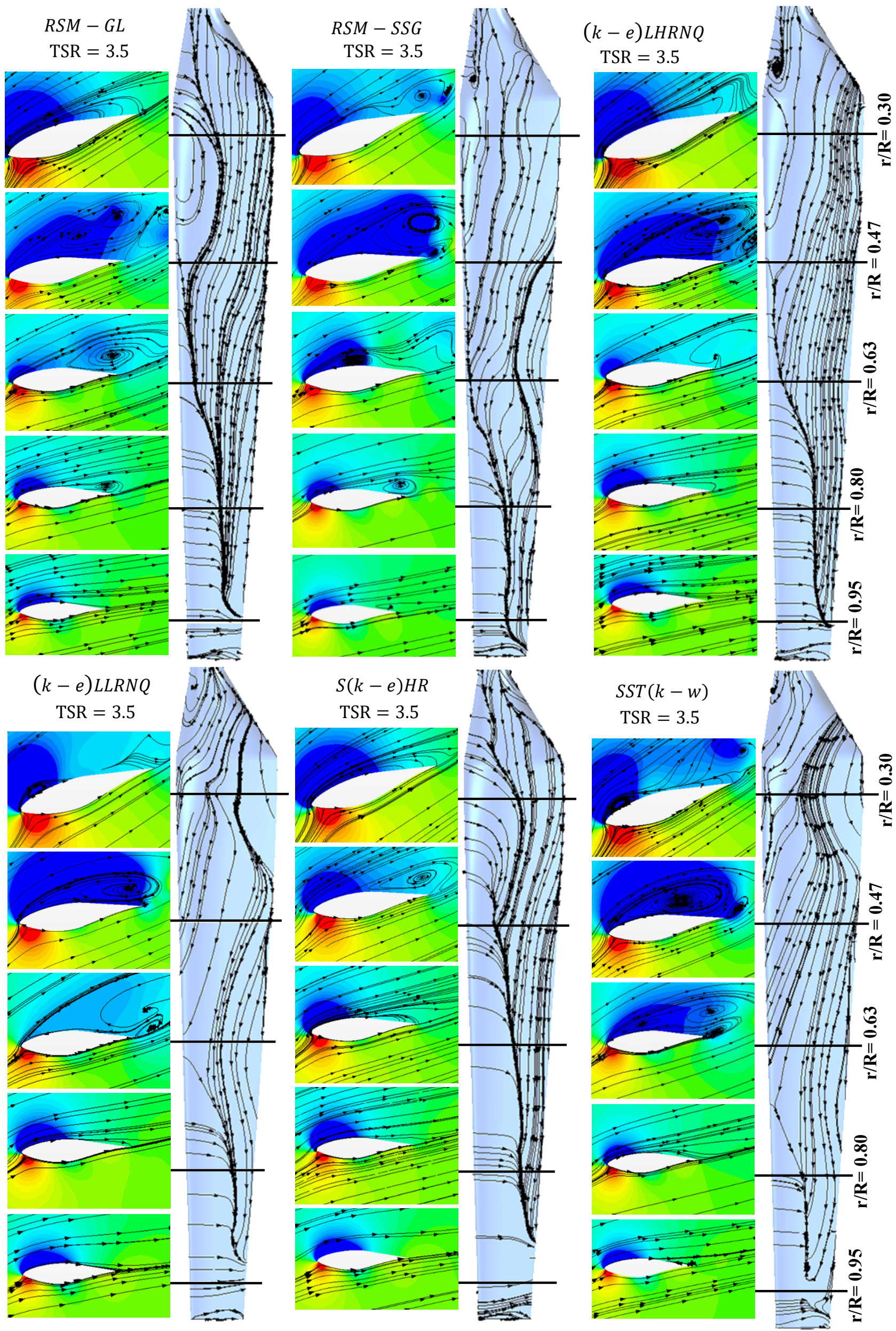

Figures 16: Instantaneous flow path lines on the upper (downwind) side of the blade and five spanwise sections for tip-speed ratio 3.5 and six turbulence models at rotational Reynolds-number $1.31 \times 10^{7}$. 

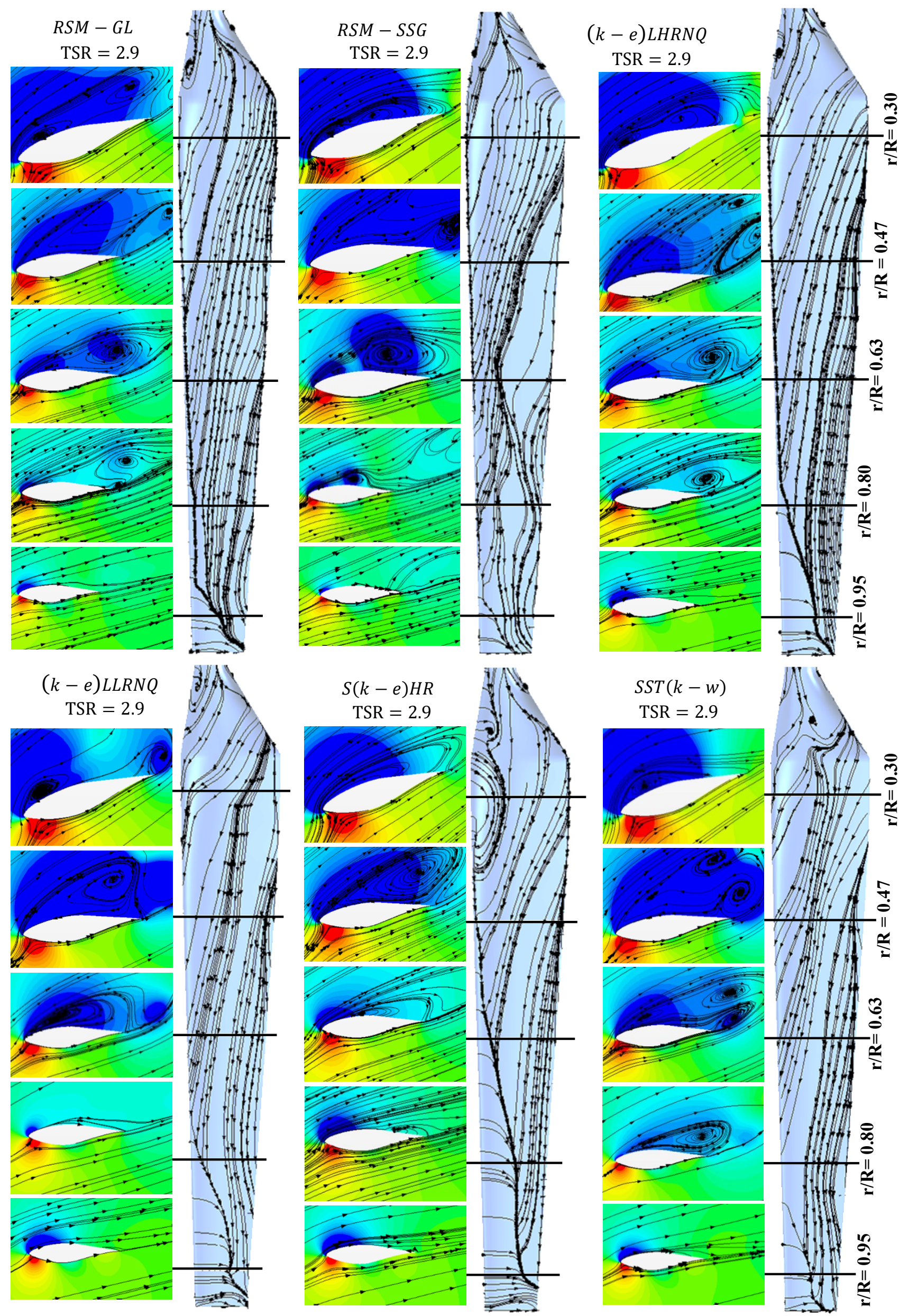

Figures 17: Instantaneous flow path lines on the upper (downwind) side of the blade and five spanwise 

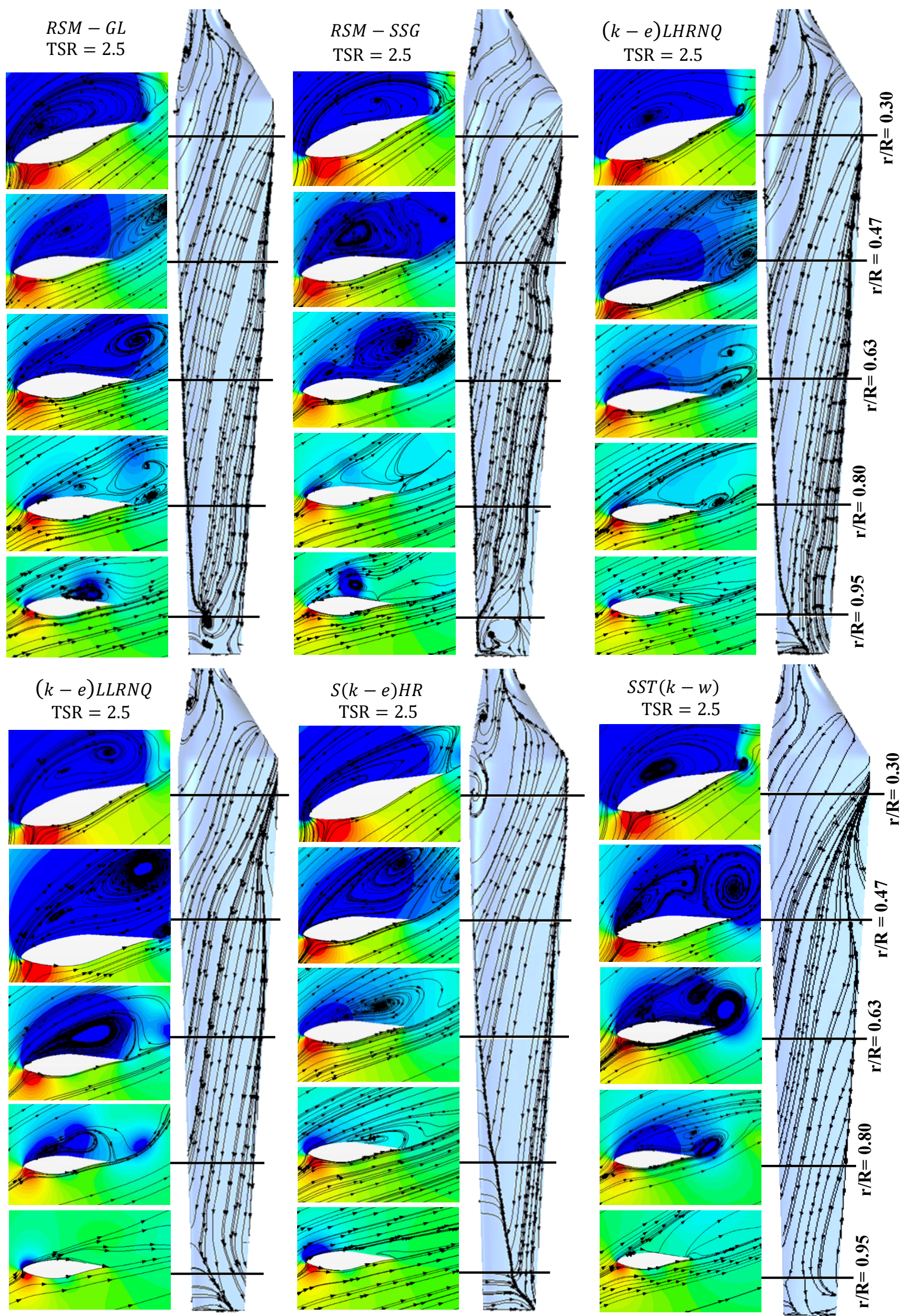

Figures 18: Instantaneous flow path lines on the upper (downwind) side of the blade and five spanwise sections for tip-speed ratio 2.5 and six turbulence models at rotational Reynolds-number $1.31 \times 10^{7}$.

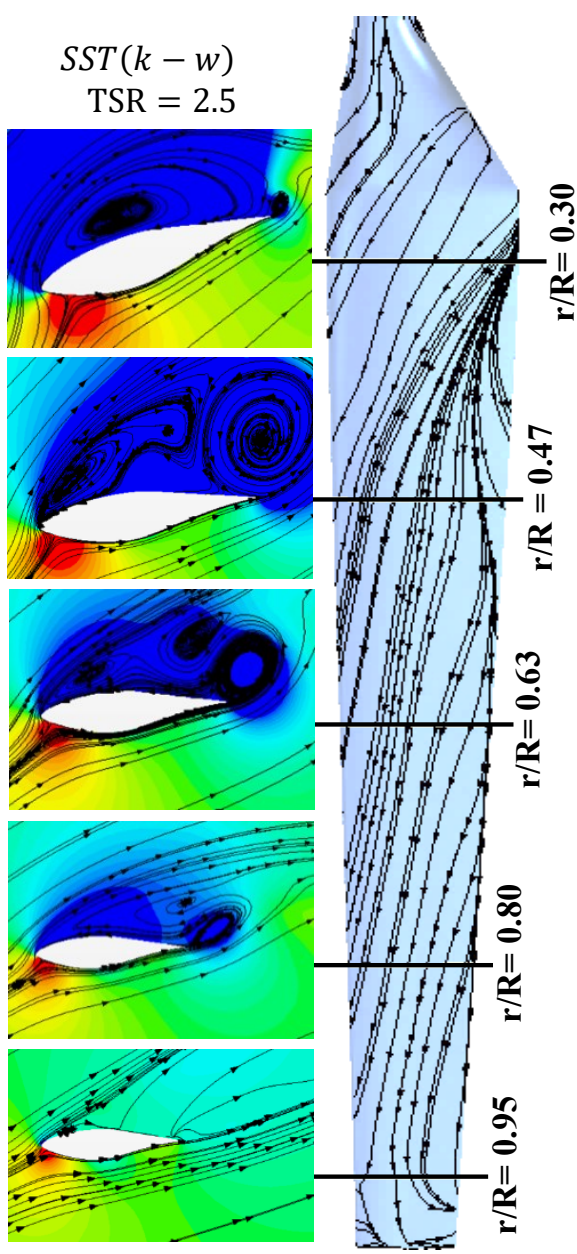



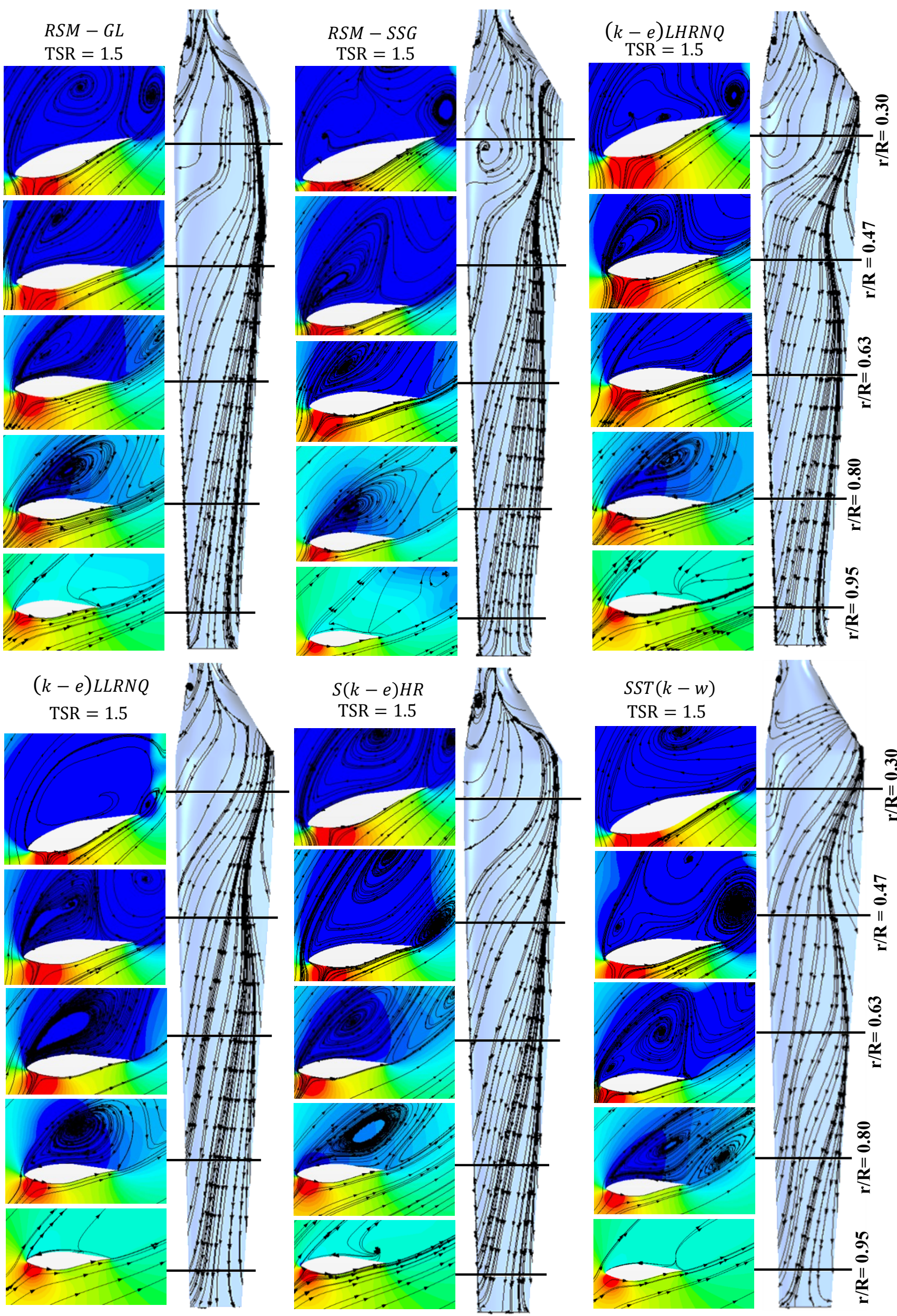

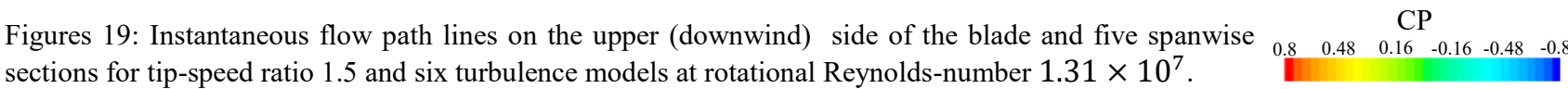




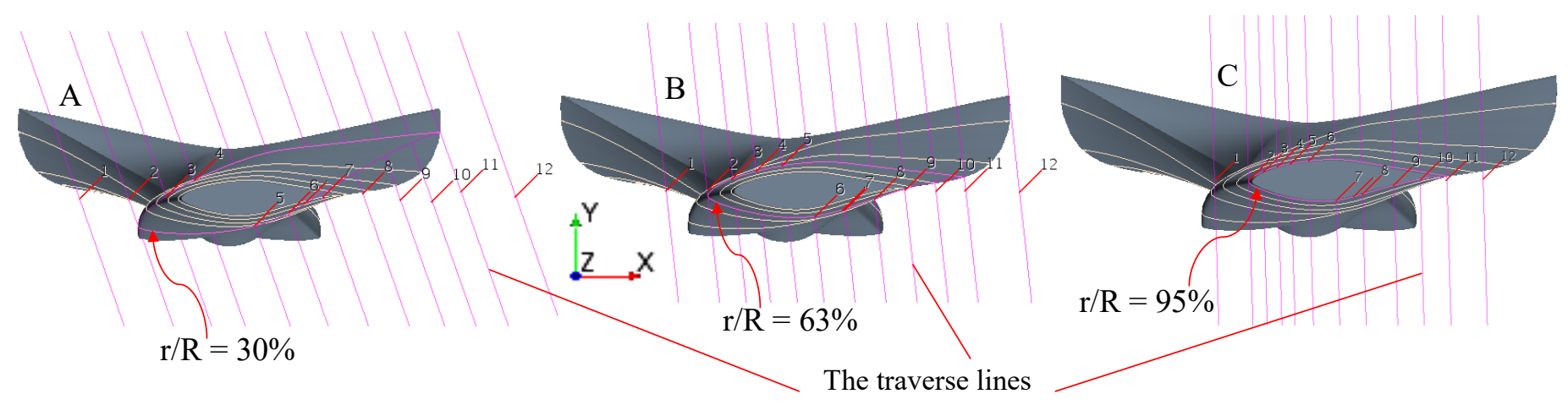

Figure 20: Views from the tip of the rotor blade show traverse lines within planes normal to the blade surface at three sections (A) $r / R=30 \%$, (B) $r / R=63 \%$, and (C) $r / R=95 \%$. 

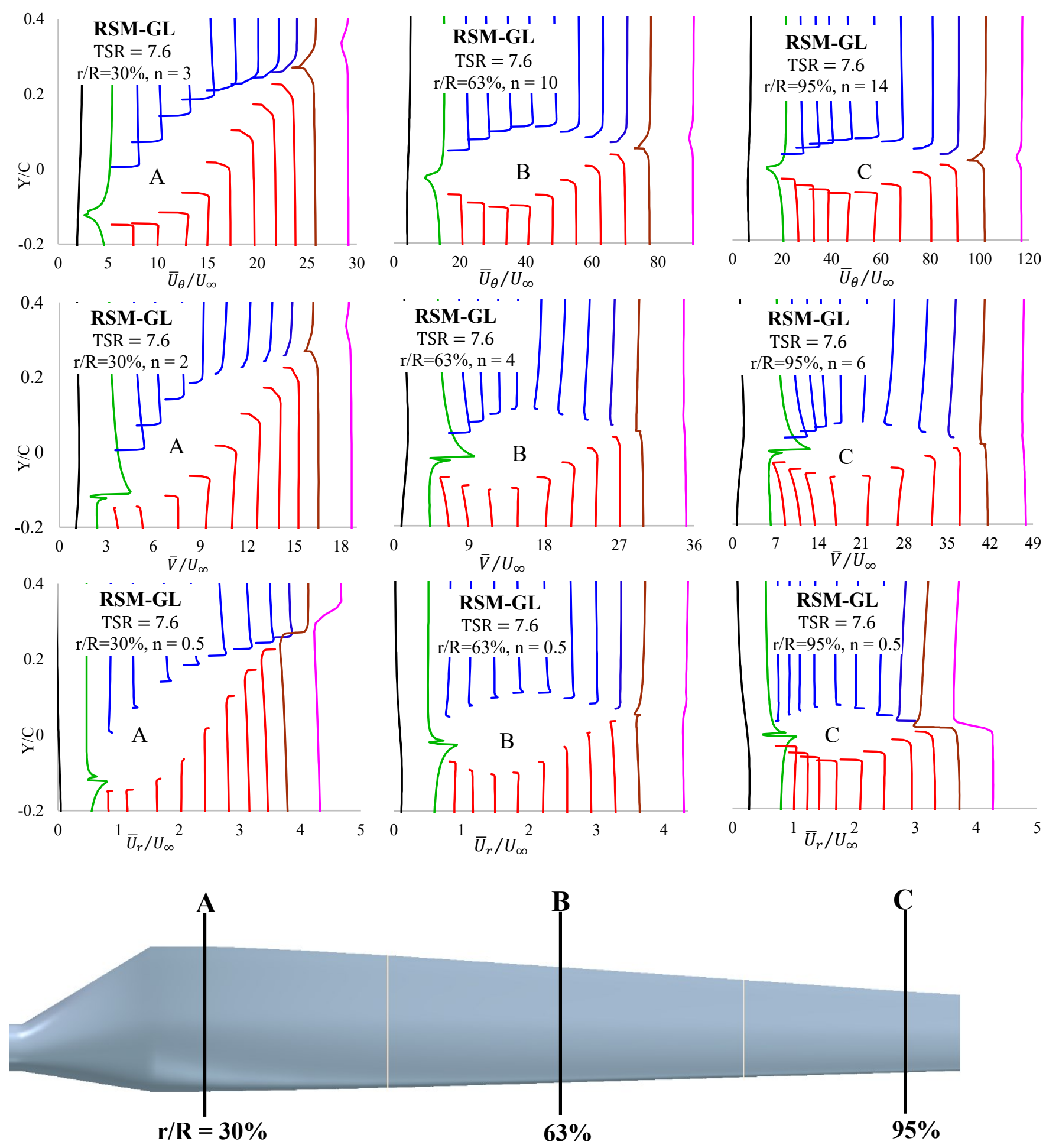

Figure 21: Profiles of the normalised relative (to the rotating blade) velocity at three sections (A) $r / R=30 \%$, (B) $63 \%$, and (C) $95 \%$ using RSM-GL for tip-speed ratio 7.6 and rotational Reynolds-number $\boldsymbol{R e}_{\boldsymbol{\theta}}=\mathbf{1 . 3 1} \times \mathbf{1 0}^{\mathbf{7}}$. 

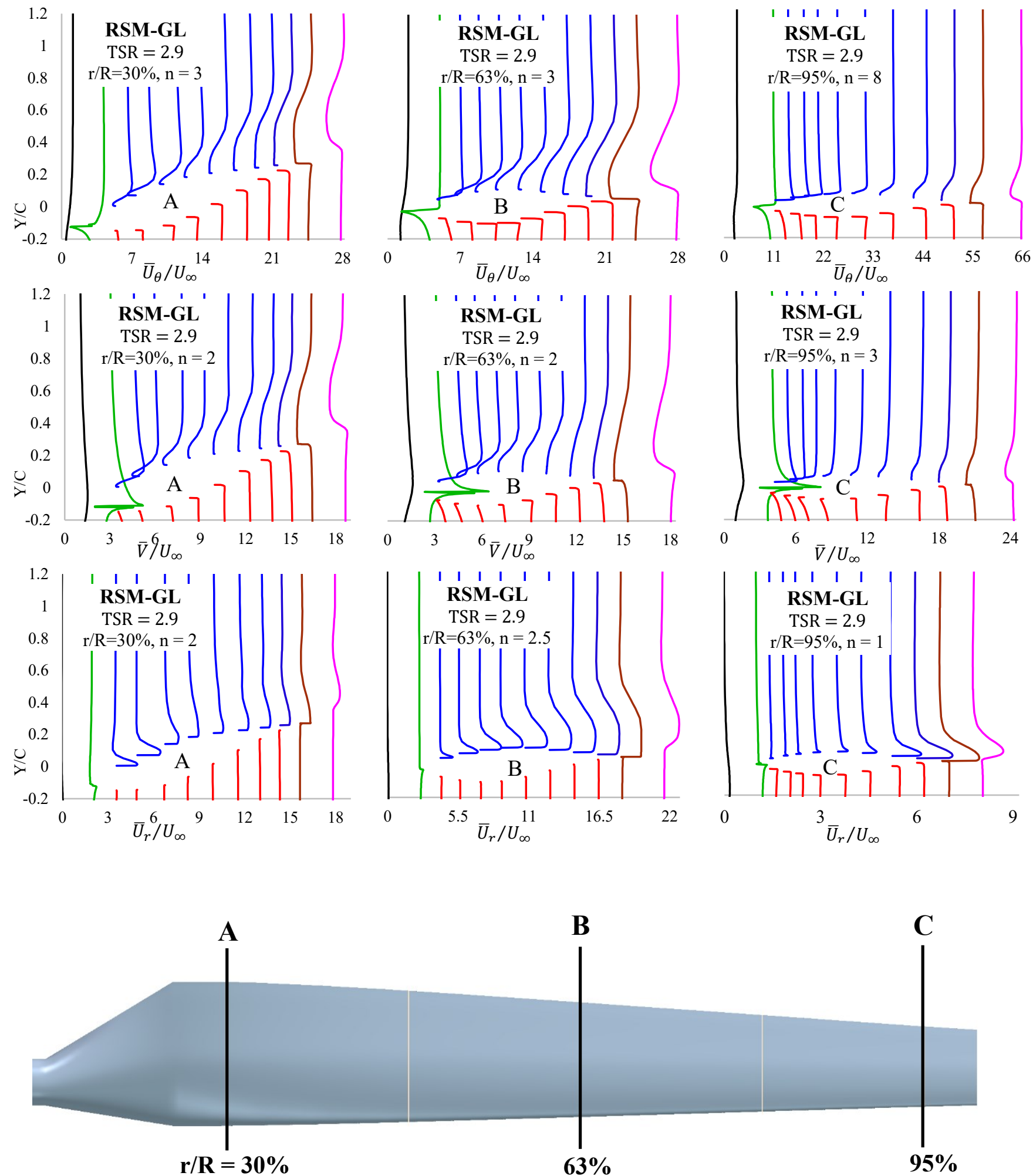

Figure 22: Profiles of the normalised relative (to the rotating blade) velocity at three sections (A) $r / R=30 \%$, (B) $63 \%$, and (C) 95\% using RSM-GL for tip-speed ratio 2.9 and rotational Reynolds-number $\boldsymbol{R e}_{\boldsymbol{\theta}}=\mathbf{1 . 3 1} \times \mathbf{1 0}{ }^{\mathbf{7}}$. 

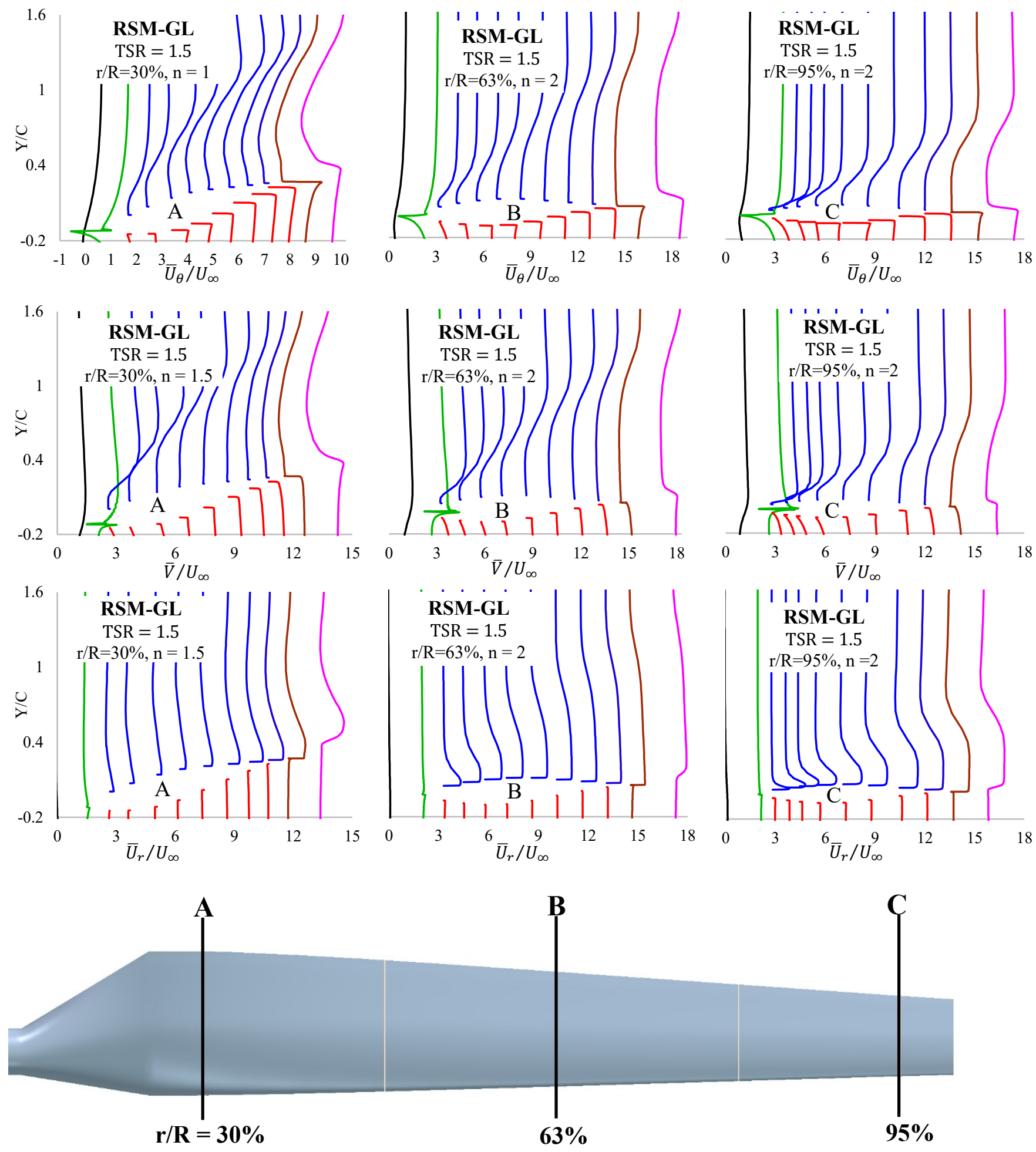

Figure 23: Profiles of the normalised relative (to the rotating blade) velocity at three sections (A) r/R=30\%, (B) $63 \%$, and (C) $95 \%$ using RSM-GL for tip-speed ratio 1.5 and rotational Reynolds-number $1.31 \times 10^{7}$. 

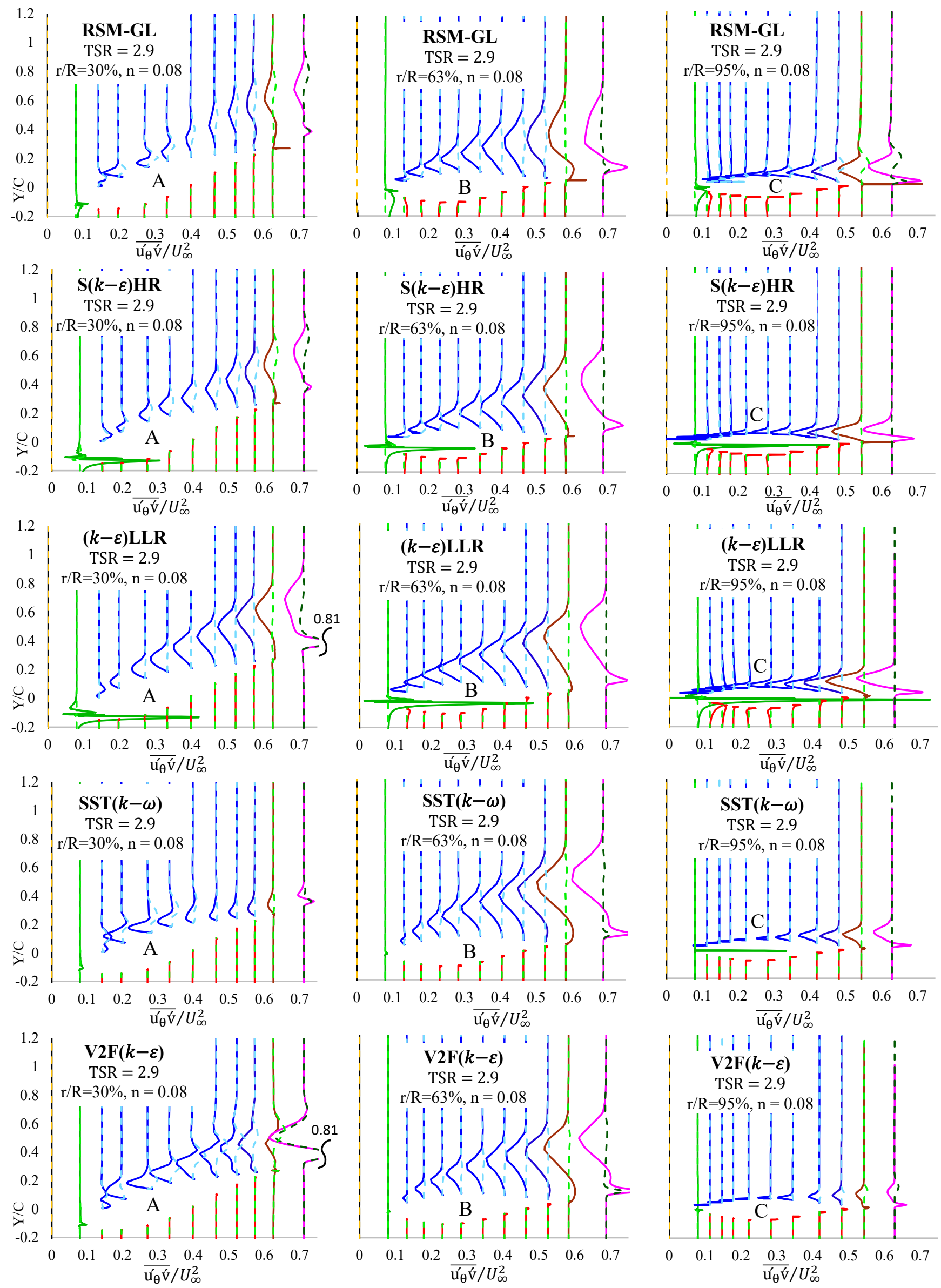

Figure 24: Normalised total (continuous curves) and resolved (broken curves) shear stresses $\overline{u_{\theta} \hat{v}} / U_{\infty}^{2}$ profiles at three sections (A) $\mathrm{r} / \mathrm{R}=30 \%$, (B) $63 \%$, and (C) $95 \%$ for various models compared to the corresponding RSM-GL for tip-speed ratio 2.9 and $R e_{\theta}=1.31 \times 10^{7}$. 

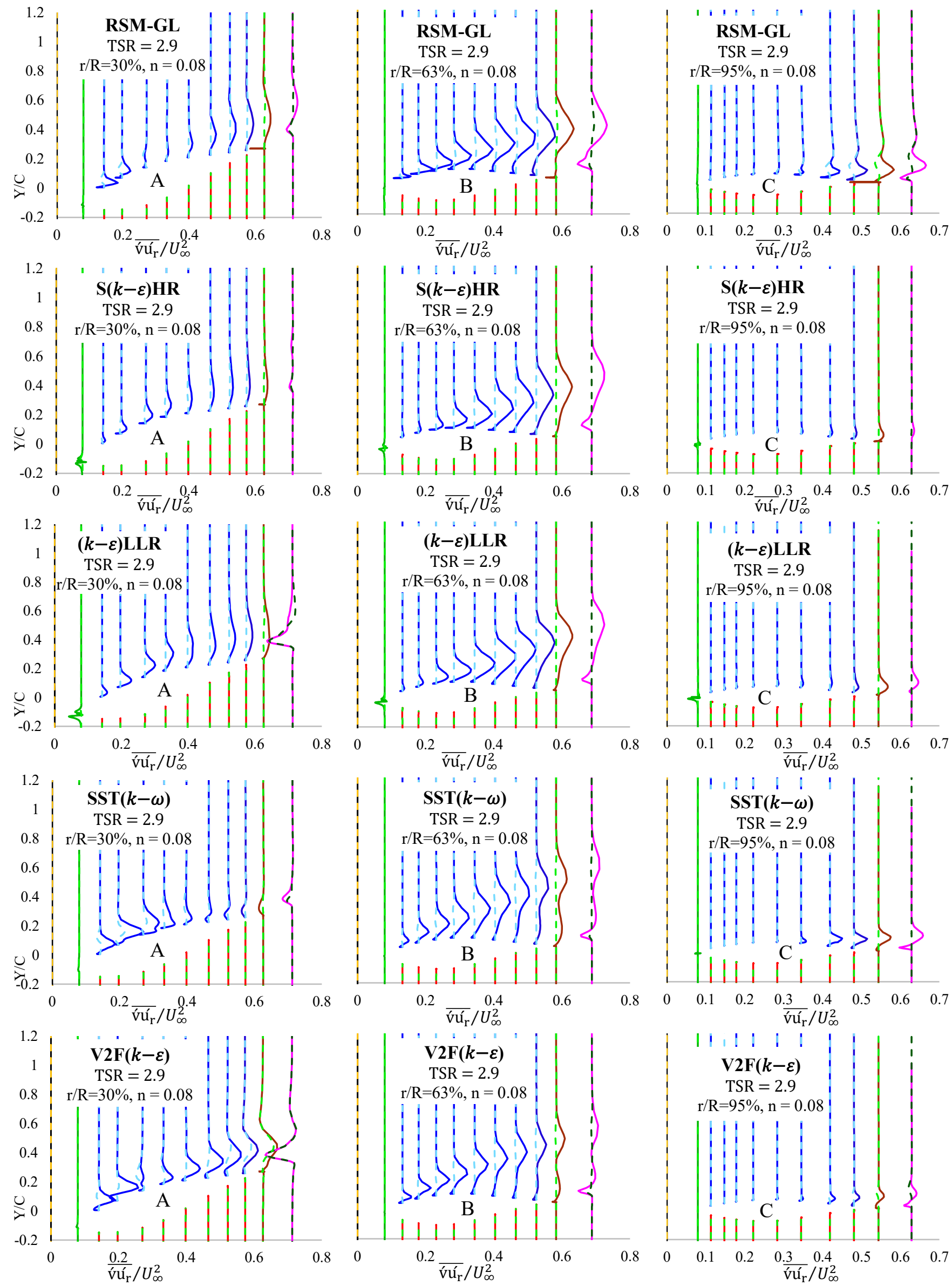

Figure 25: Normalised total (continuous curves) and resolved (broken curves) shear stresses $\overline{\mathrm{u}_{\theta} u_{\mathrm{r}}^{\prime}} / U_{\infty}^{2}$ profiles at three sections (A) $\mathrm{r} / \mathrm{R}=30 \%$, (B) $63 \%$, and (C) $95 \%$ for various models compared to the corresponding RSM-GL for tip-speed ratio 2.9 and $R e_{\theta}=$ $1.31 \times 10^{7}$. 

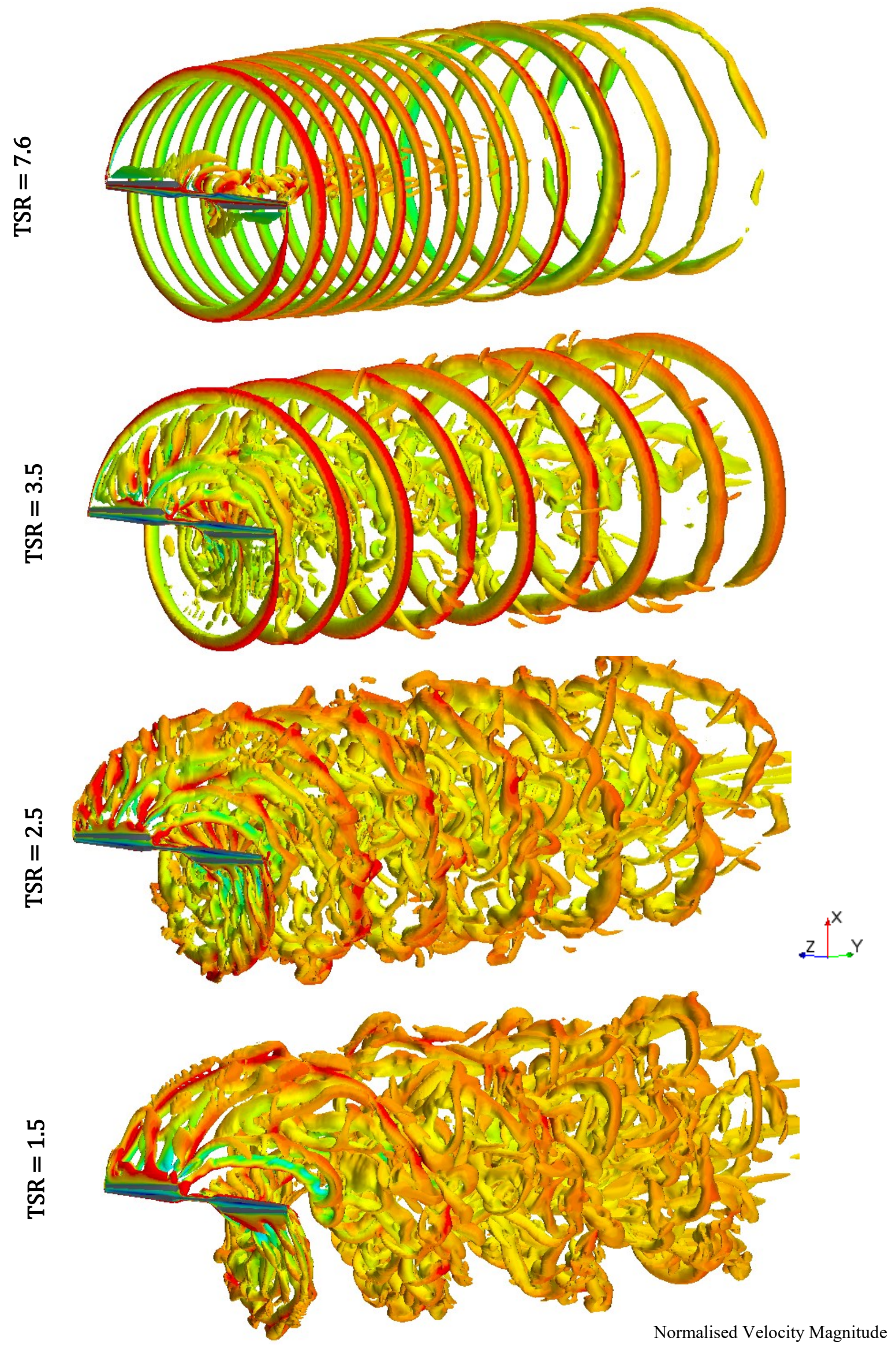

Figure 26: Comparison of vortex structures based on isosurfaces of $Q$-criteria $(Q=5.0)$ by GL-RSM models at four tip-speed ratios and rotational Reynolds-number $1.31 \times 10^{7}$.

$\begin{array}{lllll}0 & 0.285 & 0.570 & 0.855 & 1 .\end{array}$ 

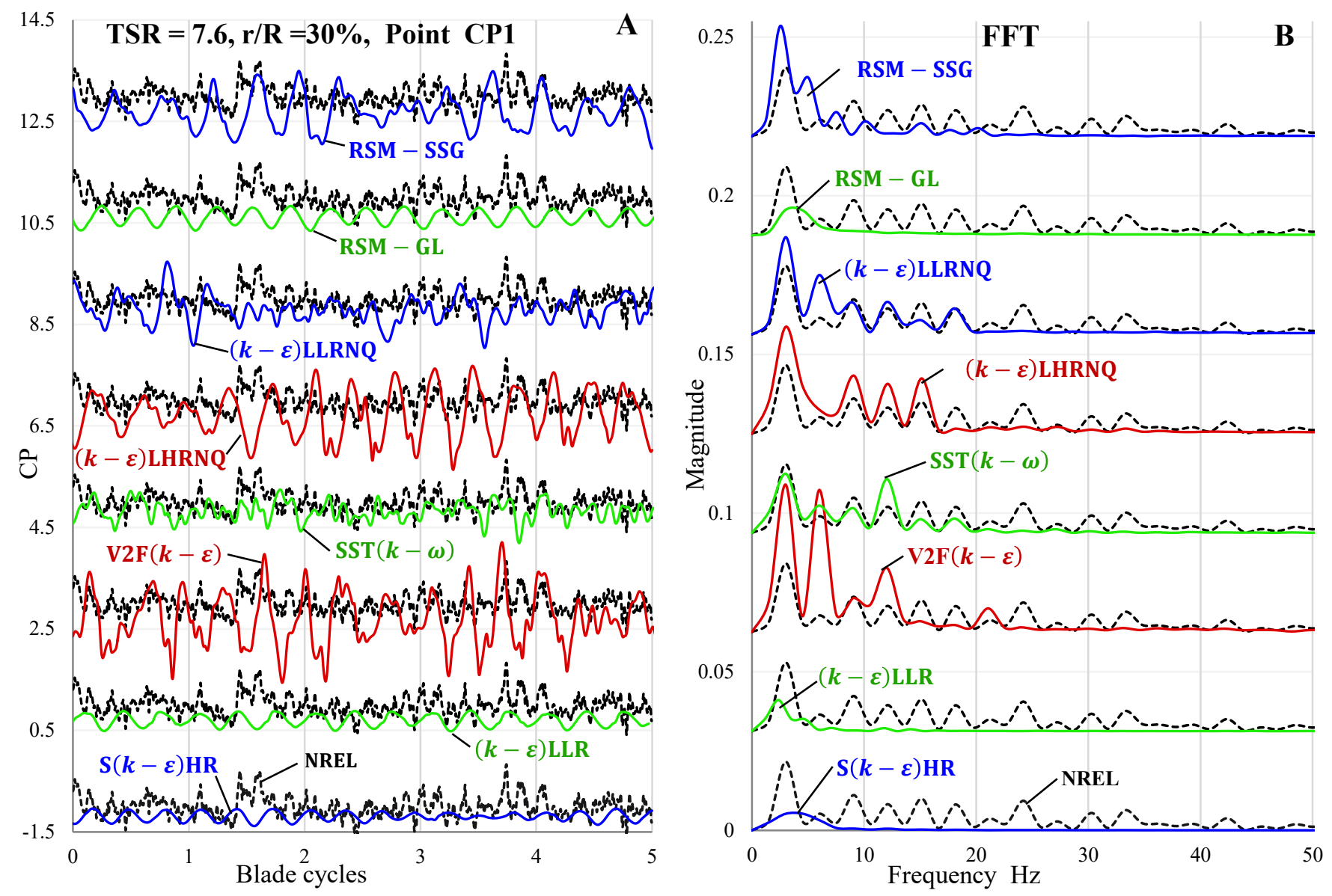

Figure 27: (A) CP transient with (B) coresponding frequency spectra at TSR $=7.6$ for point $\mathrm{CP} 1$ on the trailing-edge of section $\mathrm{r} / \mathrm{R}=30 \%$ using different turbulence models.

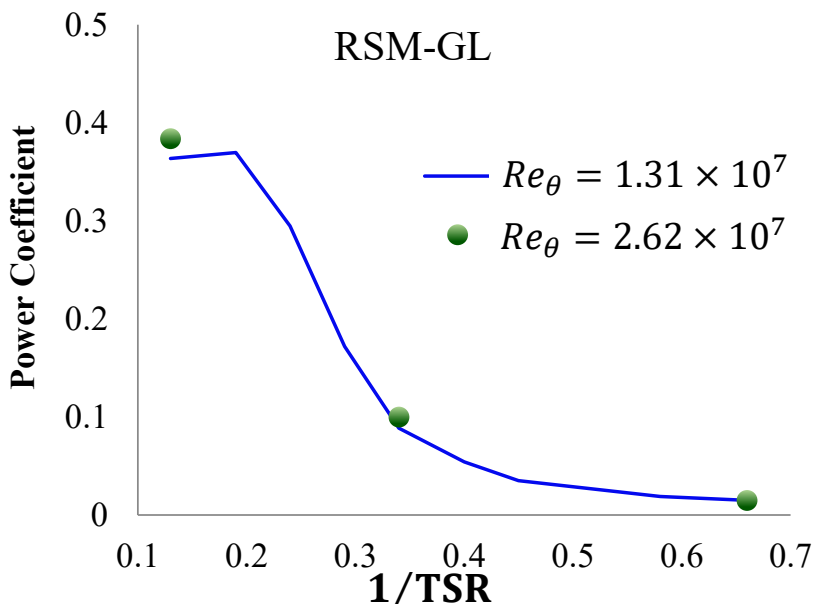

(a) Power Coefficient

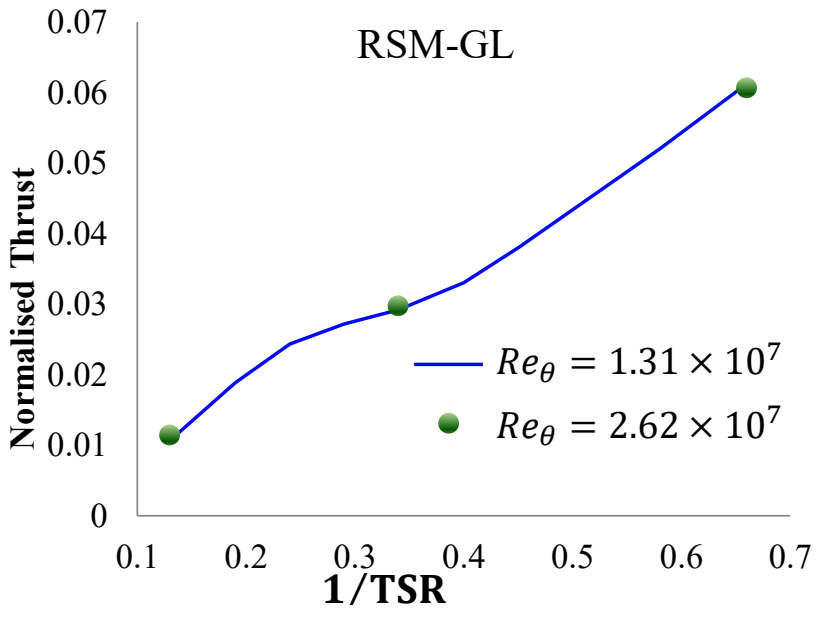

(b) Normalised Thrust

Figure 28: Comparisons of power coefficient and normalised at $R e_{\theta}=1.31 \times 10^{7}$ and $R e_{\theta}=2.62 \times 10^{7}$. 

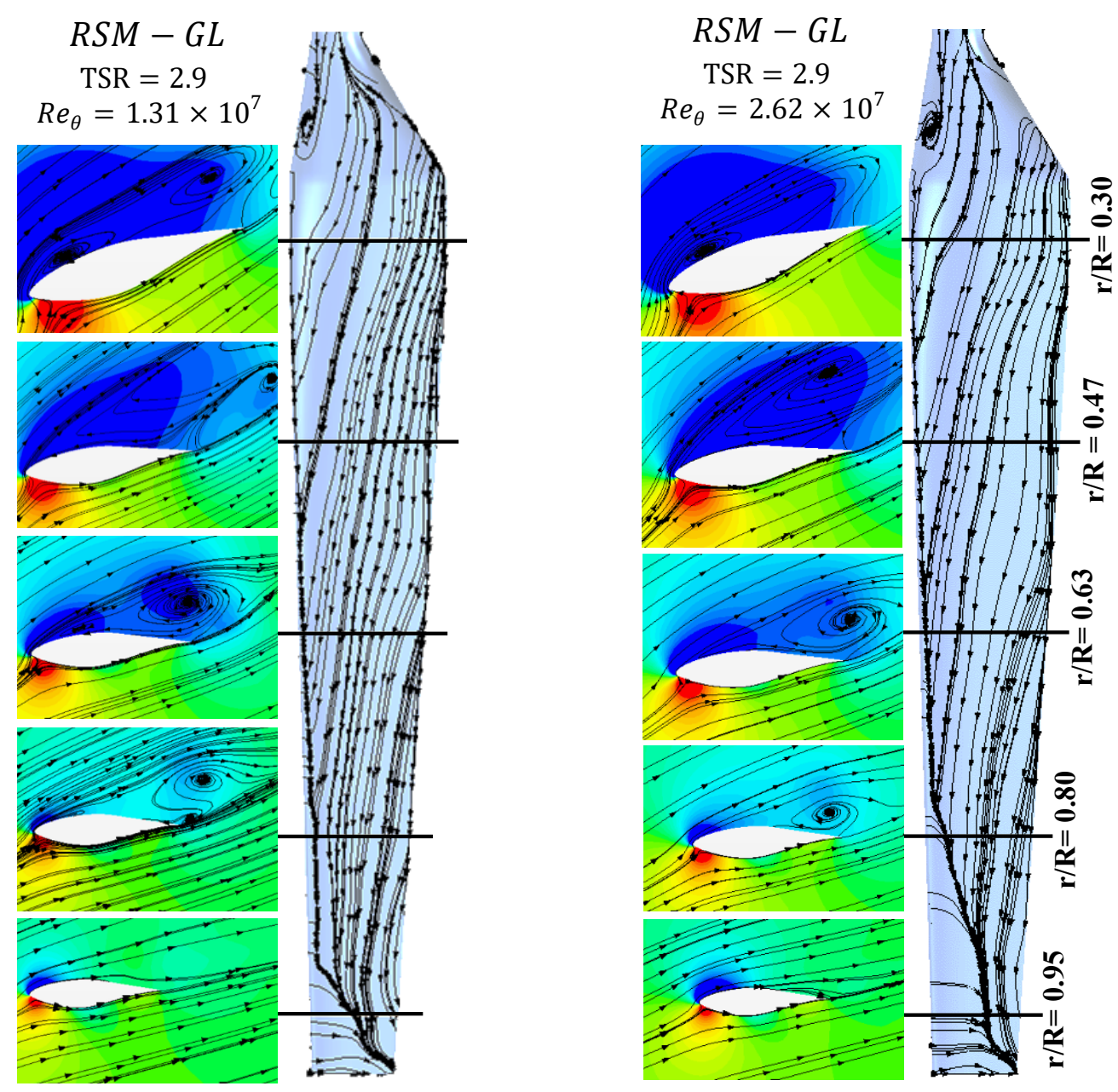

CP

$\begin{array}{llllll}0.8 & 0.48 & 0.16 & -0.16 & -0.48 & -0.80\end{array}$

Figure 29: Comparisons of flow field around the blade at $R e_{\theta}=1.31 \times 10^{7}$ and $R e_{\theta}=2.62 \times 10^{7}$. 